\title{
Real-time classification of target borders during deep brain stimulation surgery
}

Thesis for the degree of

"Doctor of Philosophy"

By

Dan Valsky

Submitted to the Senate of the Hebrew University of Jerusalem

February 2021 


\section{Real-time classification of target borders during deep brain stimulation surgery}

Thesis for the degree of

"Doctor of Philosophy"

By

Dan Valsky

Submitted to the Senate of the Hebrew University of Jerusalem

February 2021 
PhD Thesis, Dan Valsky

This work was carried out under the supervision of Prof. Hagai Bergman 
Acknowledgments

Over the course of my studies and research at the Edmond and Lily Safra Center for Brain Science (ELSC) at the Hebrew University, I have had the privilege of working with truly talented individuals. Each academic and non-academic encounter has shaped my life, and I would like to name only a few of the people who have played such a meaningful role in this journey.

I am grateful to my Ph.D. advisors Prof. Hagai Bergman and Prof. Zvi Israel, who taught me about life, disease, and science. First and foremost, I express my heartfelt thanks to Prof. Hagai Bergman. Since the time he invited me into his lab and allowed to stand shoulder to shoulder with him in the operating room for more than 350 DBS surgeries, I have been amazed by Hagai's dedication, enthusiasm and commitment to electrophysiology. For Hagai, understanding the mechanisms of the basal ganglia is the main pathway to understanding the working of this universe. I am in awe of both his intellectual abilities and his skillful management capabilities. Hagai sowed in me the first seeds of his scientific dedication and dissuaded me from becoming an engineer. He changed the way I read, write and think. By advising me on how to choose the right words, and the appropriate structure of my dissertation, he taught me how to make an enormous difference in conveying ideas and developing arguments. Hagai encouraged me to get involved in long distance running and finish two Jerusalem marathons; in both I made it to the finish line right behind him. I am extremely lucky to have found my way to the basal ganglia lab and have learned a lot from Hagai's motivation and mentorship. Hagai, you are an endless source of knowledge, ideas and guidance and, I am forever indebted to you.

I am thankful to Prof. Zvi Israel for demonstrating one of the smoothest hand motions when working under a patient's skull. Despite Zvi's professionalism in the operating room, he always displayed genuine of humanism and immense dedication to the wellbeing of both the patients and his team in the operating room. Zvi encouraged me to ride a road bike, usually behind him, and to climb the hills of Jerusalem during the hot summers, cold winters in the early mornings and late at night. After several years of working together, Zvi entrusted me with lecturing medical students on deep brain stimulation surgeries and intraoperative electrophysiology when Prof. Bergman was abroad. Zvi has always been approachable, knowledgeable and insightful.

I would also like to express my gratitude to ELSC, its leaders and sponsors. It has been a privilege to be a student in this world-class Ph.D. program and nurturing environment. I am thankful to Prof. Eli Nelken who was both a teacher and mentor. Eli combines rigorous research and flawless teaching, thus setting the bar high for students at ELSC. I thank Prof. Tali Tishby for teaching me his Information Bottleneck theory, information processing and his theories of neuroscience.

I would like to thank the patients for their cooperation and consent to be part of this research. I also thank my friends in the basal ganglia laboratory -- Alex Kaplan, Aviv Mizrahi, Marc Deffains, Pnina Rappel, Eitan Schechtman, Halen Baker, Jing Guang, Orilia Ben Yishay, Shai Heiman-Gros, Nir Ash, Salam Oukal, Omer Naor, Odaya Marmor and Boris Rosin. In particular, I would like to thank Alex Kaplan for the meaningful friendship, for all the hours of insightful discussions while running together hundreds of miles in and around Jerusalem, for his dedication to science, and for telling me what he really thought. I am thankful to Aviv 
Mizrahi for wonderful tips, discussions, constant availability and great ideas. Aviv is one of the most exuberant people dedicated to science whom I have ever had the privilege of meeting. I am grateful to Lior Tiroshi for her true friendship, her scientific curiosity, long discussions and razor-sharp advice on both academic and non-academic matters. I am thankful to Marc Deffains for interesting discussions on the stationarity of the striatal signals, and for constructive and fruitful partnership, which contributed enormously to this thesis and my overall PhD. I am thankful to Johannes Niediek for the true friendship and fruitful discussions about the mechanisms of the brain and the stationarity of recorded signals. I thank Omer Dauber (z"l) for his inquiries about the raw data and his ability to listen, which shaped this thesis. I also thank Avrama Blackwell, whose scientific guidance, support and strong encouragement extended beyond the boundaries of this thesis.

I must also thank my mother Mira Schatz, my guardian angel who rescued me from hell and made me everything that I am today. Finally, I must thank my amazing wife and ever-present collaborator, Ora Fandina. My doctorate would not have been possible without her support. Ora dedicated countless hours to helping me formulate the ideas that form the core of the thesis. Her mathematical curiosity kept my writing in check and her patience was second to none during my endless rehearsal of the presentations. Most importantly, Ora kept me sane through all the tribulations. 


\section{ABSTRACT}

Human patients with advanced Parkinson's disease (PD) and dystonia are often treated by Deep Brain Stimulation (DBS), which can alleviate the clinical symptoms of the disease. The most common brain targets are the subthalamic nucleus (STN) and the globus pallidus internus (GPi). These targets have been proven to be surgically safe and beneficial over time.

Achieving a positive therapeutic response without adverse side effects depends to a large extent on the DBS lead location. Many medical centers use electrophysiological mapping techniques; i.e., multiple (1-5) track microelectrode recordings (MER) to optimize target selection during the implantation of DBS leads. The three MER criteria for implantation into the STN and GPi are the length of the recorded trajectory in the nucleus, the presence of movement-responsive neurons, and the span of the therapeutic window which is usually defined as the distance from the borders of the adjacent axonal tracts. Currently, the demarcation of the borders and subdomains of DBS targets must be done manually by a neurophysiologist. The process of delineation of the STN-substantia nigra pars reticulata (SNr) and the striato-pallidal transitions along pre-planned trajectories is time-consuming and may be biased, leading to a sub-optimal location of the DBS lead. Several previous studies developed detection procedure to discriminate the STN from the white matter (Moran et al., 2006; Zaidel et al., 2009). Part one of my $\mathrm{PhD}$ reports the development of a real-time machine learning algorithm for the classification of both the uninterrupted STN-SNr transition (Valsky et al., 2017) and striatopallidal borders (Valsky et al., 2020).

Striatal dysfunctions play a key role in the pathophysiology of PD and dystonia, but current understanding of the changes in the discharge rate and pattern of striatal projection neurons (SPNs) remains limited. The basal ganglia (BG) are often divided into low versus high frequency discharge nuclei; i.e., the striatum versus the STN, globus pallidus externus (GPe), GPi, and the SNr. Circuitry models of PD are based on striatal dopamine loss and aberrant striatal inputs to the BG network. To date, striatal activity has not been extensively studied during electrophysiology-guided mapping in patients undergoing DBS procedures primarily because of unsuitable mapping techniques or surgical time restrictions on recordings in the striatum.

By analyzing the spatial and spectral characteristics of the striatum, GPe and GPi MERs, we discovered that pathological beta-oscillations comprised a continuous stretch within the GPi in PD (Valsky et al., 2020). This contrasts with the GPe in PD that forms gradient beta-oscillations as a function of the distance from the ventral border. We then observed that the spatial distribution of low-frequency activity for both the GPe and GPi was nearly identical across genetic and non-genetic dystonia patients. In contrast, the GPe and GPi in PD exhibited both tremor $(4-5 \mathrm{~Hz})$ and beta frequency oscillations. We discovered a similar striatal spatial oscillatory distribution across the PD, genetic and non-genetic dystonia patients.

After pooling data across all subjects and averaging across electrode trajectories, we found a unique signature in the striatum of both the non-genetic and genetic dystonia classes under light anesthesia consisting of an alpha (8-12 Hz) oscillation (Valsky et al., 2020). In contrast, the striatum in awake genetic and awake non-genetic dystonia patients displayed no oscillations. We then observed a peak in the high beta range (20-30 Hz) of the GPi in the non-genetic awake dystonia patients. In contrast, the power spectra of the GPi in the genetic dystonia patients in 
both the lightly anesthetized and awake patients displayed decreased beta-oscillatory activity. These properties contribute to the goal of identifying the accurate DBS lead placement in an automatic and unbiased way.

There is an ongoing debate in the literature as to whether the spiking activity of SPNs is grossly abnormal, as reported by Singh et al., (2016) in humans and monkeys, or not, as reported by several groups in mice and monkeys (Deffains et al., 2016a; Ketzef et al., 2017; Maltese et al., 2019b). To better understand striatal firing abnormalities, in part two of my research, I recorded and examined striatal firing abnormalities in PD and dystonic patients undergoing DBS surgeries (Valsky et al., 2020). I utilized a machine learning algorithm to automatically detect the striato-GPe border, and applied objective methods for the identification of striatal units and the quantification of their isolation quality and stationarity. Contrary to long-standing assumptions, I found no evidence for SPNs dysfunction, and that spike sorting errors were the likely cause of the perceived changes in neuronal firing rates in previous studies. The results showed that the firing rates and firing patterns of striatal neurons in PD patients did not differ significantly from those in patients with dystonia. Although some disease-dependent differences were initially observed in firing rate and burst firing, these disappeared when the analysis was restricted to well-isolated stationary recordings (Valsky et al., 2020).

Overall, the findings from this $\mathrm{PhD}$ thesis reveal that machine learning methods can provide an accurate confirmation of the STN-ventral and striato-pallidal borders without requiring input from an expert physiologist. These algorithms can thus minimize operating time and lead to outcome optimization in both STN and GPi DBS surgery. This machine learning method also helps resolve the long-standing controversy over the nature of striatal dysfunction in PD and suggests that changes in BG pathophysiology likely arise from structures outside the striatum. 


\section{Letter of contribution}

This dissertation is made up of three publications that summarize research carried out primarily by Dan Valsky, under the supervision of Prof. Hagai Bergman. The three articles were published in peer-reviewed journals. Dan Valsky is the lead author in all the three, which are listed below.

I. Designing a method for the automatic, fast and reliable detection of the subthalamic borders

Valsky, D., Marmor-Levin, O., Deffains, M., Eitan, R., Blackwell, K. T., Bergman, H., \& Israel, Z. (2017). Stop! border ahead: Automatic detection of subthalamic exit during deep brain stimulation surgery. Movement disorders : official journal of the Movement Disorder Society, 32(1), 70-79. https://doi.org/10.1002/mds.26806

II. Designing a real-time striato-pallidal border detection method

Valsky, D., Blackwell, K. T., Tamir, I., Eitan, R., Bergman, H., \& Israel, Z. (2020). Real-time machine learning classification of pallidal borders during deep brain stimulation surgery. Journal of neural engineering, 17(1), 016021. https://doi.org/10.1088/1741-2552/ab53ac

III. Determining the true discharge rate and pattern of the striatal projection neurons in Parkinson's disease and dystonia

Valsky, D., Heiman Grosberg, S., Israel, Z., Boraud, T., Bergman, H., \& Deffains, M. (2020). What is the true discharge rate and pattern of the striatal projection neurons in Parkinson's disease and dystonia? eLife, 9, e57445. Advance online publication. https://doi.org/10.7554/eLife.57445 


\section{Contents}

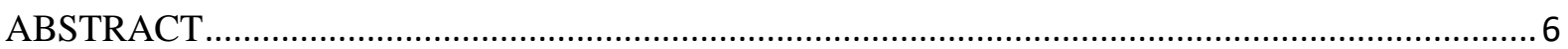

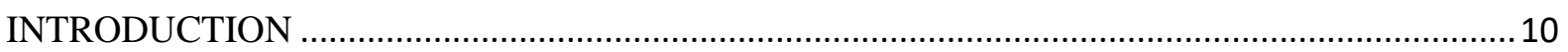

I. Pathophysiology of Parkinson's disease and dystonia ........................................................... 10

II. Clinical symptoms and treatments for Parkinson's disease ............................................... 10

III. Deep brain stimulation for treating motor symptoms of PD and dystonia ............................... 10

IV. Process for locating the DBS target borders and nearby structures........................................ 10

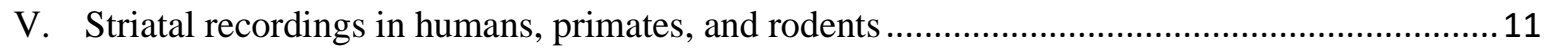

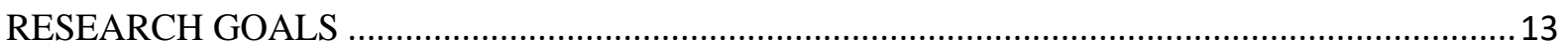

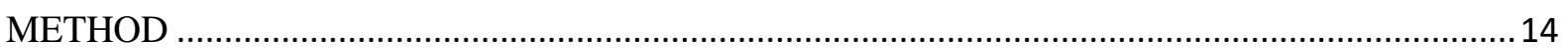

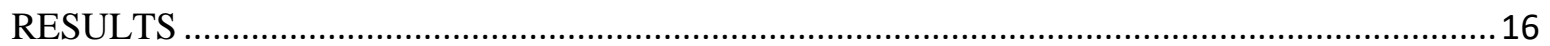

I. Designing a method for the automatic and reliable detection of the subthalamic borders .........16

II. Designing a real-time striato-pallidal border detection method ............................................. 17

III. Determining the true discharge rate and pattern of the striatal projection neurons in Parkinson's

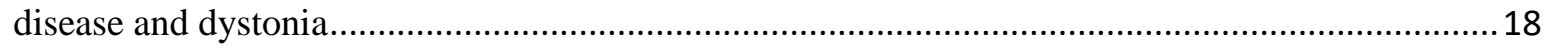

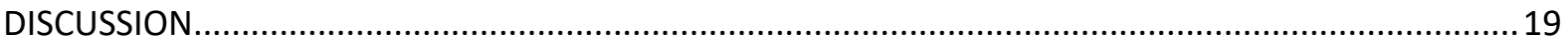

APPENDICES

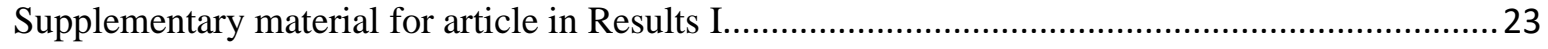

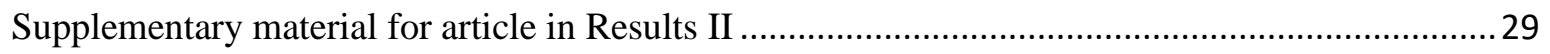

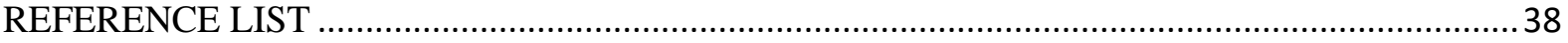




\section{INTRODUCTION}

\section{Pathophysiology of Parkinson's disease and dystonia}

Parkinson's disease (PD) and dystonia are two of the most common movement disorders, and have a wide spectrum of etiologies and clinical presentations. To date, the pathophysiology of PD and dystonia is still unclear. Traditionally, PD is attributed to the degeneration of the midbrain dopaminergic neurons which also disrupts other neuromodulary systems, including the cholinergic, serotoninergic and histaminergic systems (Bolam and Ellender, 2016; Fox et al., 2009). Although there is no degeneration of the midbrain dopaminergic neurons in dystonia, an imbalance between the midbrain dopaminergic and striatal cholinergic systems (Aosaki et al., 2010; Benarroch, 2012; Bonsi et al., 2011), as well as cerebellar dysfunction (Fremont et al., 2017; Helmich, 2018; Tewari et al., 2017) are present in both PD and dystonia. Nevertheless, BG dysfunction is still regarded as the source of the cardinal symptoms of both diseases (Mink, 1996; Wichmann, 2018).

\section{Clinical symptoms and treatments for Parkinson's disease}

Parkinson's disease (PD) is the most common basal ganglia (BG) movement disorder, affecting more than $1 \%$ of the elderly population (Bennett et al., 1996). Four motor symptoms constitute the main deficits in PD: slowness of movement (bradykinesia and akinesia), tremor, rigidity, postural instability and gait deficits. Other deficits (e.g., cognitive and emotional) also frequently accompany the motor symptoms of PD and its treatment (Dubois and Pillon, 1997; Murray, 1996). The conventional treatment of PD consists of dopamine replacement therapy, which includes dopamine precursors (e.g., levodopa), dopamine agonists as well as dopamine reuptake and catabolic inhibitors. However, after several years, severe side effects caused by this medical treatment usually develop (such as on-off fluctuation and levodopa induced dyskinesia), and patients become candidates for treatment with an invasive surgical procedure.

\section{Deep brain stimulation for treating motor symptoms of PD and dystonia}

Deep brain stimulation (DBS) of the subthalamic nucleus (STN) and the internal segment of the globus pallidus ( $\mathrm{GPi}$ ) is currently the most widespread and effective surgical procedure for advanced PD (Anderson et al., 2005; Odekerken et al., 2016; Okun et al., 2009; Weaver et al., 2012) and dystonia (Coubes et al., 2004; Krause et al., 2004; Ostrem and Starr, 2008; Vidailhet et al., 2005; Volkmann et al., 2012), with over 150,000 patients treated worldwide (Saenger et al., 2017). Implanting the DBS lead in the optimal location is critical, in that recent data from North American databases suggest that the rate of revision and removal of DBS leads is between 15.2 and $34.0 \%$, whereas up to $48.5 \%$ of revisions are due to sub-optimally placed electrodes (Rolston et al., 2016).

\section{Process for locating the DBS target borders and nearby structures}

Mis-localization of the electrodes in the structures surrounding the DBS target may cause unacceptable side effects (Benabid et al., 2009; Castrioto et al., 2014). Therefore, the current approved methods to locate the DBS target borders in the brain (both the STN and GPi for PD) involve the use of a combination of stereotactic imaging protocols and measurements of the electrical activity of brain cells, the assessment of the therapeutic impact of stimulation and adverse effects. Although recent imaging protocols have been able to improve targeting 
(Thompson et al., 2017), electrophysiology is still considered by many experts to be necessary to identify and verify the target's functional borders intraoperatively (Lozano et al., 2018). This is due to the inherent resolution limitations of neuroimaging, possible anatomic brain shifts during DBS surgery (Choi et al., 2018; Halpern et al., 2008; Khan et al., 2008), as well as differences in the connectivity and physiology of subdomains of the same anatomical nucleus.

As part of the standard clinical protocol, electrical data are collected from spike range (e.g., 300-6000 Hz) recordings, in a procedure called microelectrode recordings (MERs). MER is done today in discrete steps (i.e., 100, 500 and 1000 micrometers) along the route to the target. Usually, the demarcation of the STN and GPi borders is performed manually by a neurophysiologist. The exact detection of the STN-exit and striato-pallidal borders is considered difficult by many experts. Since DBS is carried out today in more than 1000 centers world-wide, there is a lack of electrophysiological expertise in many centers.

The detection of the dorsolateral region of the STN (STN entry) is based on clear-cut changes in electrical activity in the form of a sharp increase in MER activity (Moran et al., 2006; Zaidel et al., 2010). In contrast, several factors can make the electrophysiological determination of the ventral STN border more difficult. For example, an uninterrupted STN- substantia nigra pars reticulata ( $\mathrm{SNr}$ ) transition is less easy to detect because in this case there is no decrease in MER activity. In addition, the cells in the STN ventral domain have firing characteristics (reduced beta band and tremor frequency oscillations) resembling SNr cells (Bejjani et al., 2000; Benazzouz et al., 2002; Hutchison et al., 1998; Rodriguez-Oroz et al., 2001; Sterio et al., 2002). Finally, the electrophysiological determination of the STN exit can be challenging because white matter gaps in the STN may lead to erroneous early detection of STN exit (Moran et al., 2006).

Similarly, several factors can make the electrophysiological determination of the striatopallidal borders challenging, in particular the case of an uninterrupted GPe-GPi transition (Schiff et al., 2002), since there is no decrease in neural activity. In addition, the cells in the striatum may have transient episodes of high-frequency action potentials (injury potentials, provoked by movements of the microelectrode) resembling GPe cells (DeLong, 1971; Lozano and Hutchison, 2002; Vitek et al., 1998). Finally, in patients under general deep anesthesia there may be a significant suppression of both spontaneous and evoked neuronal discharges from pallidal neurons (Hutchison et al., 2003; Venkatraghavan et al., 2016). Therefore, electrophysiological determination of the STN and striato-pallidal borders can be ambiguous, leading to suboptimal location of the DBS lead and inadequate clinical outcomes.

\section{Striatal recordings in humans, primates, and rodents}

The striatum (i.e., the main input structure of the BG network) is the main recipient of midbrain dopaminergic neurons (Menegas et al., 2015) and also receives di-synaptic cerebellar projections (Bostan et al., 2013; Hoshi et al., 2005). Most [ 95\% and $~ 70-80 \%$ in rodents and primates, respectively (Graveland et al., 1985; Petryszyn et al., 2018, 2014)] striatal neurons are medium spiny projection neurons (SPNs) that receive afferents from the cortex and the thalamus, and together with the STN neurons, innervate the central (i.e., GPe) and output (i.e., GPi and SNr) BG structures (Albin et al., 1989; Gerfen et al., 1990). Therefore, alterations in striatal signaling disrupt normal BG activity and may lead to the manifestation of the motor and non-motor symptoms of PD and dystonia (Albin et al., 1989; Bergman et al., 1990; Gerfen et al., 1990). 
Dysregulation of BG activity may consist of changes in discharge rate (Albin et al., 1989; Gerfen et al., 1990). Unlike in all other BG structures, extracellular recordings of spiking activity in non-human primates (NHPs) reveal that SPNs have a very low discharge rate $(\sim 1-$ $2 \mathrm{~Hz}$ at rest) and are phasically active (i.e. they emit short bursts) around relevant behavioral events (Crutcher and DeLong, 1984; Kimura et al., 1990). However, previous studies of the BG in the NHP model of PD have mainly focused on the STN, GPe and GPi which are structures with a high frequency tonic discharge (i.e. $25-70 \mathrm{~Hz}$ at rest) (Deffains et al., 2016a; Filion and Tremblay, 1991; Wichmann and DeLong, 2003). These studies have reported excessive GPi/SNr discharge rates in PD that lead to an increase in BG inhibitory outputs to the thalamus and the frontal cortex motor areas (Wichmann and DeLong, 2003). Conversely, it is assumed that dystonia (and other hyper-kinetic states) are characterized by reduced GPi/SNr activity (Guehl et al., 2009).

More recent studies of the BG in animal (rodent and NHP) models of PD and human patients (undergoing DBS procedures) have dealt with changes in discharge patterns and synchronization. Parkinsonism-related $\beta$ oscillations have been observed in local field potentials (LFPs) recorded in all BG structures, including the striatum (Deffains et al., 2016a; Kondabolu et al., 2016; Lemaire et al., 2012; Singh and Papa, 2019). Similarly, low frequency (4-12Hz) LFP oscillations have been recorded in the BG network of dystonic patients (PiñaFuentes et al., 2018; Silberstein et al., 2003). Finally, $\beta$ oscillations are commonly observed in the spiking activity of the STN, GPe and GPi of MPTP-treated monkeys (Deffains et al., 2016a; Soares et al., 2004) and PD patients (Kühn et al., 2005; Moshel et al., 2013; Silberstein et al., 2003; Valsky et al., 2020a; Zaidel et al., 2010).

Nevertheless, direct evidence of abnormal activity of the striatal SPNs is still elusive. Previous studies utilizing the NHP model of PD reported striking increases $(\sim 15$-fold increase from the normal discharge rate of $\sim 1-2 \mathrm{~Hz}$ ) in the firing rate of the SPNs subsequent to striatal dopamine depletion and the induction of parkinsonism (Singh et al., 2016). The same research group also reported a high discharge rate of SPNs recorded in PD and dystonic patients $(\sim 30 \mathrm{~Hz}$ and $9 \mathrm{~Hz}$, respectively) (Singh et al., 2016). They also found a significant change in the firing pattern of striatal neurons, with many SPNs exhibiting bursting activity in PD patients and MPTP monkeys as compared to a smaller fraction in patients with dystonia. Finally, the SPNs of patients with an essential tremor (ET, regarded as a non-BG disorder) have a very low discharge rate $(\sim 2 \mathrm{~Hz})$ and no tendency to burst, as reported in normal NHPs (Liang et al., 2008; Singh et al., 2016).

These spectacular changes in the discharge rate and pattern of SPNs in the NHP model of PD run counter results obtained in our research group. Deffains et al. recorded the activity of SPNs and other BG neurons in Vervet monkeys before and after systemic 1-methyl-4-phenyl-1,2,3,6tetrahydropyridine (MPTP) treatment and the induction of severe parkinsonian symptoms. Although Deffains et al. found robust changes in the discharge properties (rate, pattern and synchronization) of the STN, GPe and BG output structures, they did not observe any difference in the discharge rate $(\sim 2-3 \mathrm{~Hz})$ or pattern of SPNs in the MPTP-treated monkeys in comparison to the recordings in the same monkeys before MPTP treatment (Deffains et al., 2016a; Deffains and Bergman, 2019).

Extracellular recordings of SPN spiking activity of anesthetized (Mallet et al., 2006; Sharott et al., 2017) and awake (Chen et al., 2006; Kish et al., 1999) rats before and after striatal dopamine 
depletion by 6-hydroxydopamine (6-OHDA) treatment have revealed a significant, but very slight increase in the SPN discharge rate. Rodent studies make it possible to differentiate between SPNs expressing D1 and D2 dopamine receptors. A significant imbalance in the discharge rate and calcium dynamics of D1 and D2 SPNs was observed in the dopaminedepleted striatum (Mallet et al., 2006; Parker et al., 2018; Sharott et al., 2017). In particular, D2 SPNs increased their discharge (Mallet et al., 2006; Sharott et al., 2017) and were also prone to being entrained to parkinsonian $\beta$ oscillations (Sharott et al., 2017). Nevertheless, the absolute increase in the discharge rate even of the D2 SPNs was still modest (from $\sim 0.5$ to $\sim 2.8 \mathrm{~Hz}$ ) (Sharott et al., 2017). Moreover, recent studies have reported no significant increase in the low discharge rate of either SPN subpopulation in striatal dopamine-depleted mice (Ketzef et al., 2017; Maltese et al., 2019b).

Since only one research group has recorded drastic increases in the spontaneous spiking activity in the striatum of PD patients in comparison to either dystonic patients or the normal levels of striatal activity reported in healthy animals (Singh et al., 2016), we examined our human patient data to determine whether we could report similar changes.

\section{RESEARCH GOALS}

\section{Designing a method for the automatic, fast and reliable detection of the subthalamic borders}

In Part I of my $\mathrm{PhD}$ thesis, I designed an automatic method for the detection of the STNsubstantia nigra pars reticulata ( $\mathrm{SNr}$ ) transition, based on a characterization of their electrical activity. Existing methods (Danish et al., 2008; Falkenberg et al., 2006; Zaidel et al., 2009), are successful in identifying the STN-white matter transitions, but are not as reliable in identifying the STN-SNr transition. To improve the detection of STN-SNr transition as well as the lower border of the STN, I developed a computational analysis method that utilizes MER power spectra and allows for real-time, high accuracy discrimination between STN and SNr (Valsky et al., 2017).

\section{Designing a real-time striato-pallidal border detection method}

In Part II, I addressed the current need for improved MER analysis during GPi targeting. I developed a classification system to enable neurosurgeons to actively track the delineation of the pallidal borders along pre-planned trajectories. Recent work in pallidal physiological mapping (Alam et al., 2016; Neumann et al., 2017; Wang et al., 2018) has underscored the potential value of intraoperative computer-aided classification of the borders. I utilized a dataset covering five classes of disease to train the classification algorithm composed of awake Parkinson's disease patients, as well as awake and lightly anesthetized both genetic and nongenetic dystonia patients. I designed a machine learning algorithm to provide predictions of the pallidal borders, based on hidden Markov models and the $\mathrm{L}_{1}$-distance measure in RMS and power spectra of the MER. I tested the machine learning algorithm's performance prospectively against the judgment of three electrophysiologists in the operating rooms of three hospitals (Hadassah-Hebrew University Medical Center, Sourasky Medical Center, and Rabin Medical Center) using newly collected data (Valsky et al., 2020). 


\section{Determining the true discharge rate and pattern of the striatal projection neurons in Parkinson's disease and dystonia}

There is an ongoing debate in the literature as to whether the spiking activity of SPNs is grossly abnormal, as reported by Singh et al., (2016) in humans and monkeys, or not, as reported by several groups in mice and monkeys (Deffains et al., 2016a; Ketzef et al., 2017; Maltese et al., 2019b). To date, striatal activity has not been extensively studied during electrophysiologyguided mapping in patients undergoing DBS procedures primarily because of unsuitable mapping techniques or surgical time restrictions on recordings in the striatum. To better understand striatal firing abnormalities, in part III of my $\mathrm{PhD}$ thesis, I recorded and examined striatal firing abnormalities in PD and dystonic patients undergoing DBS surgeries (Valsky et al., 2020). I utilized a machine learning algorithm to automatically detect the striato-GPe border, and applied objective methods for the identification of striatal units and the quantification of their isolation quality and stationarity (Valsky et al., 2020).

\section{METHOD}

\section{Patients and surgery}

In total, 7,204 (131 trajectories, 81 patients) and 11,774 (148 trajectories, 52 patients) MER segments of background spiking activity undergoing STN and GPi DBS surgery were analyzed in this thesis. For the STN-exit detection study (Results I), the neuronal database was split into two parts. The training dataset was composed of 58 trajectories (obtained from 30 PD patients) containing 2678 stable MER segments. Seventy-three additional trajectories recorded from 51 other patients, yielding 4526 stable MER segments (test dataset) used solely to test the robustness of the HMM detection. For the striato-GPe-GPi detection study (Results II), a total of 116 microelectrode trajectories (42 PD and dystonia patients) aimed at the GPi in five classes of disease were analyzed, yielding a total of 11,774 MER segments. The five classes included: awake PD patients, awake and lightly anesthetized genetic and non-genetic dystonia patients. For the striatum study (Results III), a total of 93 microelectrode trajectories aimed at the GPi were analyzed (48 in PD and 45 in dystonia), yielding a total of 933 and 718 MER segments within the posterior putamen of patients suffering from PD and dystonia, respectively. A total of 16 PD and 13 dystonic (non-genetic and genetic dystonia) patients were involved.

All patients met the criteria for DBS and signed written informed consent for surgery that involved microelectrode recordings. The studies were authorized and approved by the Institutional Review Board of Hadassah Hospital in accordance with the Helsinki Declaration (reference code: 0168-10-HMO).

Surgery was performed using a CRW stereotactic frame (Radionics, Burlington, MA, USA). BG target coordinates were chosen as a composite of the indirect anterior commissure-posterior commissure (AC-PC) atlas-based location and direct (1.5 or 3Tesla) T2 magnetic resonance imaging, using Framelink 4 or 5 software (Medtronic, Minneapolis, USA).

\section{Data acquisition}

The data were acquired using two systems: MicroGuide (prior to 2015, previously described in (Castrioto et al., 2016)) and Neuro Omega (from 2015, Alpha Omega, Nazareth, Israel). In the Neuro Omega system, raw and spiking signals were amplified by 20 , and sampled at 44 $\mathrm{kHz}$ by a 16 -bit $\mathrm{A} / \mathrm{D}$ converter (using $\pm 1.25 \mathrm{~V}$ input range; i.e., $\sim 2-\mu \mathrm{V}$ amplitude resolution). 
The raw signal was bandpass filtered from 0.075 to $10 \mathrm{k} \mathrm{Hz}$, using a hardware 2 and 3 pole Butterworth filter, respectively. The spiking signal was bandpass filtered from 300 to $9000 \mathrm{~Hz}$, using a hardware 4-pole Butterworth filter.

In the MicroGuide system (Alpha Omega Engineering, Nazareth, Israel), neurophysiological activity was recorded via polyamide coated tungsten microelectrodes (Alpha Omega) with an impedance mean \pm the standard deviation (SD): $0.63 \pm 0.16 \mathrm{M} \Omega$ (measured at $1 \mathrm{kHz}$ at the beginning of each trajectory). The signal was amplified by 10,000 or 25,000 , band-passed from 250 to $6,000 \mathrm{~Hz}$, using a hardware four-pole Butterworth filter, and sampled at 24 or $48 \mathrm{kHz}$ by a 12 bit A/D converter (using $\pm 5 \mathrm{~V}$ input range).

\section{Microelectrode recordings}

For each recording session in the STN DBS, for both the left and right hemispheres, a MER trajectory using one or two parallel microelectrodes (2 mm apart) was made, starting $10 \mathrm{~mm}$ above the pre-operative T2 MRI image-based calculated target (center of the dorsolateral STN). Trajectories followed a double-oblique approach towards the dorsolateral STN target, which was designed to avoid the cortical sulci, the ventricles and major blood vessels. Therefore, although primarily in a dorsal-ventral direction, the trajectories also took an anterior-posterior and lateral-medial course. A typical trajectory was approximately 60 degrees from the axial AC-PC plane and 15 degrees from the mid-sagittal plane.

On each recording session in the GPi DBS, a MER exploration was made starting $15 \mathrm{~mm}$ above the calculated target. Our trajectories followed a double-oblique approach (50 to $80^{\circ}$ from the axial AC-PC plane and 0 to $10^{\circ}$ degrees from the midsagittal plane) through the posterior putamen and the GPe and towards the ventral border of the posterior-lateral portion of the GPi target. The "central" electrode was directed at the ventral border of the posterior-lateral portion of the GPi and the "anterior" electrode was located $2 \mathrm{~mm}$ anterior/ventral to the central electrode in the parasagittal plane. Some of these recordings were made by a single microelectrode trajectory (instead of two) to accommodate cortical anatomy under the burr hole and brain blood vessels.

MER segments were regularly sampled in space to avoid recording bias towards particular striatal units. For all the DBS surgeries, the step size between two MER segments ranged from 100 to $200 \mu \mathrm{m}$ and was controlled by the neurophysiologist to achieve optimal identification of the borders. At each step, MER segments were recorded from 4 to 140s (after a 2-s signal stabilization period).

\section{Software and Statistics}

In this thesis, the striatal, GPe, GPi and STN MERs were subjected to root mean square (RMS), power spectral density (PSD) analysis, offline spike sorting, support vector machine (SVM) and cluster analysis, as well as advanced methods such as a hidden Markov model (HMM). These techniques are presented in the method sections of the respective articles (Results I- III). 


\section{RESULTS}

I. Designing a method for the automatic and reliable detection of the subthalamic borders

a. Valsky, D., Marmor-Levin, O., Deffains, M., Eitan, R., Blackwell, K. T., Bergman, H., \& Israel, Z. (2017). Stop! border ahead: Automatic detection of subthalamic exit during deep brain stimulation surgery. Movement disorders : official journal of the Movement Disorder Society, 32(1), 70-79. https://doi.org/10.1002/mds.26806 


\title{
Stop! Border Ahead: Automatic Detection of Subthalamic Exit During Deep Brain Stimulation Surgery
}

\author{
Dan Valsky, MS, ${ }^{1,2 *}$ Odeya Marmor-Levin, MS, ${ }^{2}$ Marc Deffains, PhD, ${ }^{2}$ Renana Eitan, MD, ${ }^{3}$ Kim T. Blackwell, VMD, PhD, ${ }^{4}$ \\ Hagai Bergman, MD,PhD, ${ }^{1,2}$ and Zvi Israel, MD ${ }^{5}$ \\ ${ }^{1}$ The Edmond and Lily Safra Center for Brain Research (ELSC), The Hebrew University, Jerusalem, Israel \\ ${ }^{2}$ Department of Medical Neurobiology (Physiology), Institute of Medical Research - Israel-Canada (IMRIC), The Hebrew University-Hadassah \\ Medical School, Jerusalem, Israel \\ ${ }^{3}$ Department of Psychiatry, Hadassah-Hebrew University Medical Center, Jerusalem, Israel \\ ${ }^{4}$ Krasnow Institute for Advanced Study, George Mason University, Fairfax, Virginia, USA
}

${ }^{5}$ Center for Functional \& Restorative Neurosurgery, Department of Neurosurgery, Hadassah-Hebrew University Medical Center, Jerusalem, Israel

\begin{abstract}
Background: Microelectrode recordings along preplanned trajectories are often used for accurate definition of the subthalamic nucleus (STN) borders during deep brain stimulation (DBS) surgery for Parkinson's disease. Usually, the demarcation of the STN borders is performed manually by a neurophysiologist. The exact detection of the borders is difficult, especially detecting the transition between the STN and the substantia nigra pars reticulata. Consequently, demarcation may be inaccurate, leading to suboptimal location of the DBS lead and inadequate clinical outcomes.

Methods: We present machine-learning classification procedures that use microelectrode recording power spectra and allow for real-time, high-accuracy discrimination between the STN and substantia nigra pars reticulata.

Results: A support vector machine procedure was tested on microelectrode recordings from 58 trajectories that included both STN and substantia nigra pars reticulata that achieved a $97.6 \%$ consistency with
\end{abstract}

human expert classification (evaluated by 10-fold crossvalidation). We used the same data set as a training set to find the optimal parameters for a hidden Markov model using both microelectrode recording features and trajectory history to enable real-time classification of the ventral STN border (STN exit). Seventy-three additional trajectories were used to test the reliability of the learned statistical model in identifying the exit from the STN. The hidden Markov model procedure identified the STN exit with an error of $0.04 \pm 0.18 \mathrm{~mm}$ and detection reliability (error $<1 \mathrm{~mm}$ ) of $94 \%$.

Conclusions: The results indicate that robust, accurate, and automatic real-time electrophysiological detection of the ventral STN border is feasible. (C) 2016 International Parkinson and Movement Disorder Society.

Key Words: subthalamic nucleus; substantia nigra; deep brain stimulation; Parkinson's disease; microelectrode recording ${ }^{*}$ Correspondence to: Dan Valsky, MS, Department of Medical Neurobi-
ology (Physiology), The Hebrew University-Hadassah Medical School,
POB 12272, Jerusalem, 91120, Israel; dan.valsky@mail.huji.ac.il
Relevant conflicts of interest/financial disclosures: There is no conflict
of interest on the part of any of the authors.

Funding agencies: This study was supported by the Magnet program of the Office of the Chief Scientist (OCS) of the Israel Ministry of Economy. Contract grant sponsor: NIAAA; Contract grant number: R01 016022. Contract grant sponsor: NIDA; Contract grant number: R01 038890.

Received: 10 April 2016; Revised: 8 August 2016; Accepted: 24 August 2016

Published online 6 October 2016 in Wiley Online Library (wileyonlinelibrary.com). DOI: 10.1002/mds.26806
Surgical treatment for advanced Parkinson's disease (PD) includes high-frequency deep brain stimulation (DBS) of the subthalamic nucleus (STN), which has proved to be surgically safe and beneficial over time. ${ }^{1-}$ ${ }^{4}$ In some patients, mood disorders such as depression ${ }^{5}$ or manic symptoms ${ }^{6-11}$ may be observed after stimulation as a result of suboptimally placed DBS leads. By contrast, the combined stimulation of the substantia nigra pars reticulata $(\mathrm{SNr})$ and $\mathrm{STN}$ may improve freezing of gait in patients with advanced PD. ${ }^{10,11}$ Therefore, accurate differentiation of the STN from the $\mathrm{SNr}$ is essential for achieving optimal therapeutic benefit while avoiding psychiatric complications. 
Microelectrode recordings (MERs) along preplanned trajectories are often used for improved delineation of the location of the STN during DBS surgery for Parkinson's disease. The detection of the dorsolateral region of the STN is based on clear-cut changes in electrical activity in the form of a sharp rise in the total power of the MER (as measured by the root mean square [RMS]), ${ }^{12}$ the tremor frequency, and the $\beta$ oscillatory activity $(13-30 \mathrm{~Hz}){ }^{13}$ In contrast, several factors can make electrophysiological determination of the ventral STN border more difficult, in particular, an uninterrupted STN-SNr transition, because in this case there is no drop in activity (or RMS). In addition, the cells in the STN ventral domain have firing characteristics (reduced $\beta$-band and tremor-frequency oscillations) resembling SNr cells. ${ }^{14-18}$ Finally, electrophysiological determination of the STN exit can be challenging because white matter gaps in the STN may lead to erroneous early detection of STN exit. ${ }^{12}$ Therefore, the electrophysiological determination of the STN ventral border can be ambiguous and occasionally difficult to define.

Although recent imaging studies have been able to improve the differentiation between the STN and the $\mathrm{SNr},{ }^{19}$ electrophysiology is still necessary to identify and verify the STN-SNr transition intraoperatively. To facilitate detection of the transition, this article describes a new automatic, reliable procedure for locating the STN exit. Earlier automatic methods that use RMS values ${ }^{12,20-23}$ were successful in identifying STN-white matter (STN-WM) transitions, but were not as good for the direct STN-SNr transition. To improve the STN-SNr transition and STN lower border detection, we developed a computational analysis procedure that capitalizes on several features from the power spectra of the MER and allows for high accuracy discrimination between the STN and the SNr.

\section{Patients and Methods}

\section{Patients and Surgery}

MERs were analyzed from 131 microelectrode trajectories that passed through both the STN and the SNr of 81 Parkinson's disease patients undergoing bilateral STN DBS implantation. The patients' demography and clinical state were as follows: mean age, 62.1 years; mean disease duration, 10.3 years; $36 \%$ female, mean Unified Parkinson's Disease Rating Scale — part III (UPDRS III) score OFF/ON therapy before surgery, 51.1/19.4; and mean levodopa equivalent dosage before surgery, $849.6 \mathrm{mg} /$ day. Patient demographic information appears in Supporting Information Table S4. This study was authorized and approved by the Institutional Review Board of Hadassah Hospital in accordance with the Helsinki Declaration (reference code HMO-006412). All patients were awake during surgery. Further details on the surgical procedure and data acquisition can be found in our previous reports. ${ }^{12,23}$

\section{Microelectrode Recordings}

For both the left and right hemispheres, 1 or 2 parallel microelectrodes were inserted, and the recording started $10 \mathrm{~mm}$ above the calculated target. Our trajectories followed a double-oblique approach toward the dorsolateral STN target. In most cases, 2 microelectrodes were used (Fig. 1A). A "central" electrode was directed at the center of the dorsolateral STN target (as per imaging) and often traversed the STN and entered the $\mathrm{SNr}$ without passing through the white matter. An "anterior" electrode was advanced $2 \mathrm{~mm}$ anterior to the central electrode (in the parasagittal plane) and therefore crossed the STN-SNr area in a more ventral plane. In contrast to the central electrode, the anterior electrode often passed through the white matter before it entered the SNr. Analysis was not based on continuous recordings during the entire advance toward the dorsolateral STN target, but rather on segments of data recorded at specific points (without electrode movement; Fig. 1B). Segments of data were recorded for at least 4 seconds after $0.5 \mathrm{sec}$ onds of lowering the electrode. Further details on the microelectrode recordings and the intervals of the depths are presented as Supporting Information.

\section{Neural Data Sets}

We divided our neuronal database into 2 parts. The training data set was composed of 58 trajectories (obtained from 30 PD patients) containing 2678 stable MERs recorded in the white matter before the STN, STN dorsolateral oscillatory region (DLOR), STN ventromedial nonoscillatory region (VMNR), and white matter after STN and SNr. A subset of this data set, containing 1720 MERs from the dorsal and ventral STN as well as the SNr, was used for the support vector machine (SVM) procedure. The training data set of 58 trajectories was also used to find the optimal parameters for the hidden Markov model (HMM). Seventy-three additional trajectories recorded from 51 other patients, and yielding 4526 stable MERs (test data set), were used solely to test the robustness of the HMM detection.

\section{Root Mean Square}

The RMS estimate was calculated from the multiunit activity recorded by the microelectrode at each electrode depth. RMS values are susceptible to electrode properties (eg, electrode impedance $)^{12}$; hence, the RMS was normalized by the pre-STN (white matter) baseline RMS, ${ }^{12,23}$ creating what we term the normalized RMS (NRMS). 
$\left.\begin{array}{lll}V A L S Y & \text { E T }\end{array}\right]$

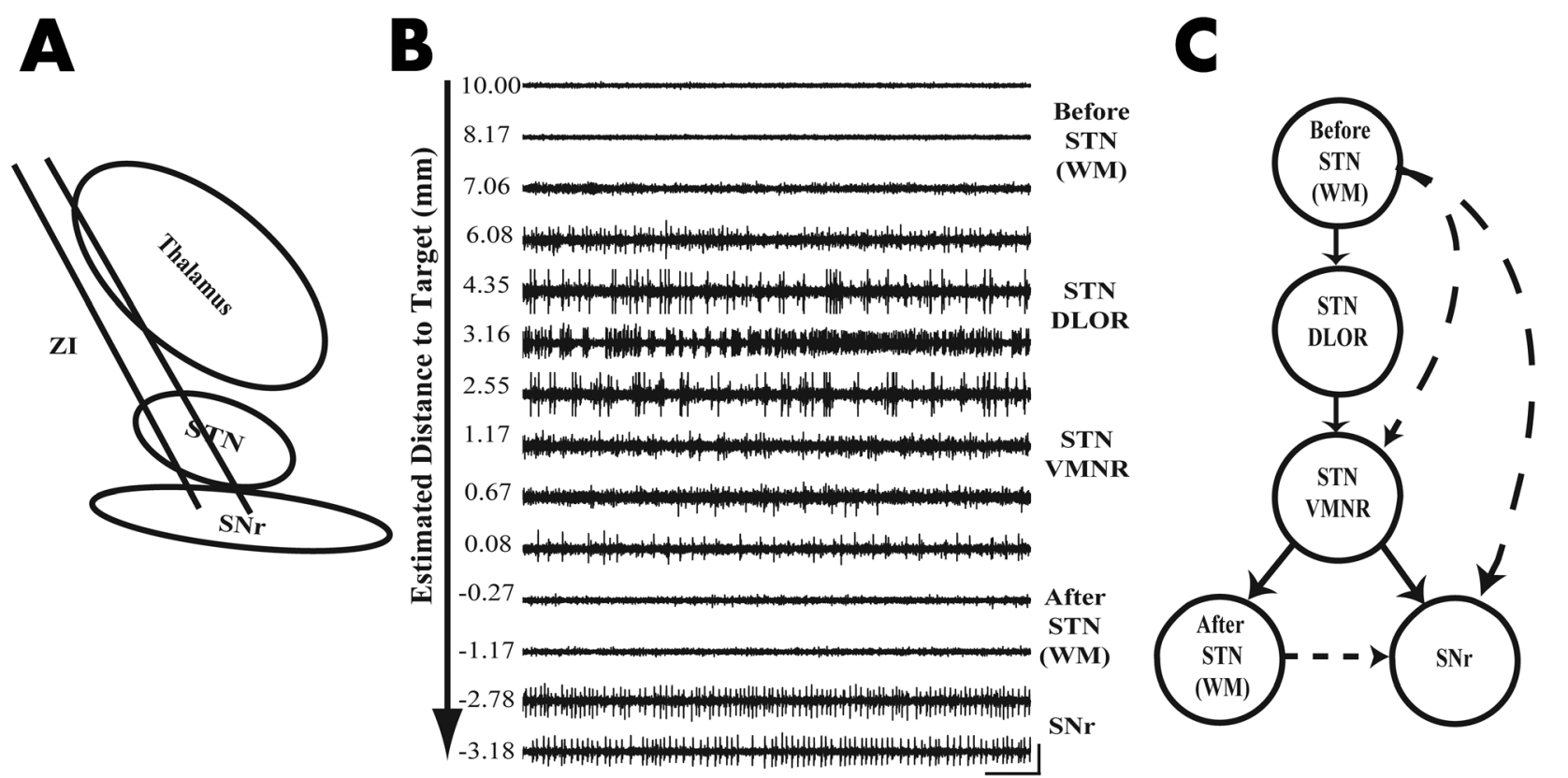

FIG. 1. Overview of STN targeting. (A) Schematic diagram of a typical trajectory of two parallel microelectrodes showing subcortical structures. STNSubthalamic nucleus, SNr- Substantia nigra reticulata, ZI - zona incerta (B) One-second example of 14 representative raw signal traces (in descending order) out of the 82 recorded signal traces at various depths along the trajectory (a single DBS track) from a Parkinson's disease patient. The signal at each position was recorded for at least 4 seconds. The segment at $2.55 \mathrm{~mm}$ estimated distance to target (EDT) was recorded for a longer period of time than the others because responses to active and passive limb movements are tested. The traces indicate regions of internal capsule (white matter), dorsalateral oscillatory region (DLOR) STN, ventral medial non oscillatory region (VMNR) STN, white matter between STN and SNr; vertical bar indicates $256 \mu \mathrm{v}$, horizontal bar indicates $100 \mathrm{~ms}$. (C) Functional state model represents the anatomy which is sequentially encountered during microelectrode recording of the STN detection. At all depths along the trajectory the electrode is in one of a finite set of states. Arrows between the states represent the possible state transitions. Solid lines depict a typical trajectory state sequence that proceeds through the first three states consecutively and then to the white matter between STN and SNr. Dotted lines depict: 1) a trajectory that enters the SNr immediately after the STN, 2) a trajectory that starts in the white matter and enters the VMNR, 3) one that goes directly into the SNr without passing through the STN.

\section{Power Spectral Density (PSD)}

Visual inspection of the average STN and SNr power spectra revealed significant differences in the $5-300 \mathrm{~Hz}$ domain. To identify the frequency band that contained the largest difference between the STN and the SNr, we divided the $5-300 \mathrm{~Hz}$ range of the power spectra into 10 approximately logarithmically spaced bands. For each band, we calculated the mean power for each MER and then evaluated the difference in the mean power between the STN and the SNr. Using this method, we identified which frequency bands had the largest difference between the STN and the SNr. Additional details are presented in the Supporting Information.

\section{SVM Discrimination of STN and SNr MERs}

In machine learning, SVMs are supervised learning models that are specifically designed to solve a classification problem off-line, after all the data have been collected. For our SVM analysis, measurements in both time and frequency domains (based on the NRMS and power spectra of the MERs) were used as features for the SVM classification. The classification procedure used the NRMS and the " $100-150 \mathrm{~Hz} / 5-25 \mathrm{~Hz}$ power ratio" features, as well as their class label (STN or $\mathrm{SNr}$ ) for each of the 1720 MERs in the training data set. The performance of the SVM classifier was evaluated by 10 -fold cross-validation. Additional details are presented in the supporting information.

The SVM requires labeling the MERs of each region, which is not amenable to real-time use. Here we used the SVM to identify which features had the most information in terms of discriminating regions. However, once the optimal features had been selected, the SVM was no longer needed or used.

\section{The Hidden Markov Model}

The HMM takes the set of features extracted from the raw data as input and provides the output clustering in real time. In previous reports, ${ }^{23,24}$ the HMM procedure was used to discriminate the STN from the white matter. This study goes beyond these previous works by designing a HMM procedure with improved ability to detect the STN exit by delineating the borders between STN and SNr (even for cases without a WM gap between the STN and the SNr). Details on the HMM are provided in the supporting information.

All statistical analyses were performed using custom-made MATLAB 7.5 routines (Mathworks, Natick, MA). The statistics presented in this report, if not specified otherwise, are the mean \pm standard error of the mean; the criterion for statistical significance was set at $P<0.05$ for all statistical tests. 

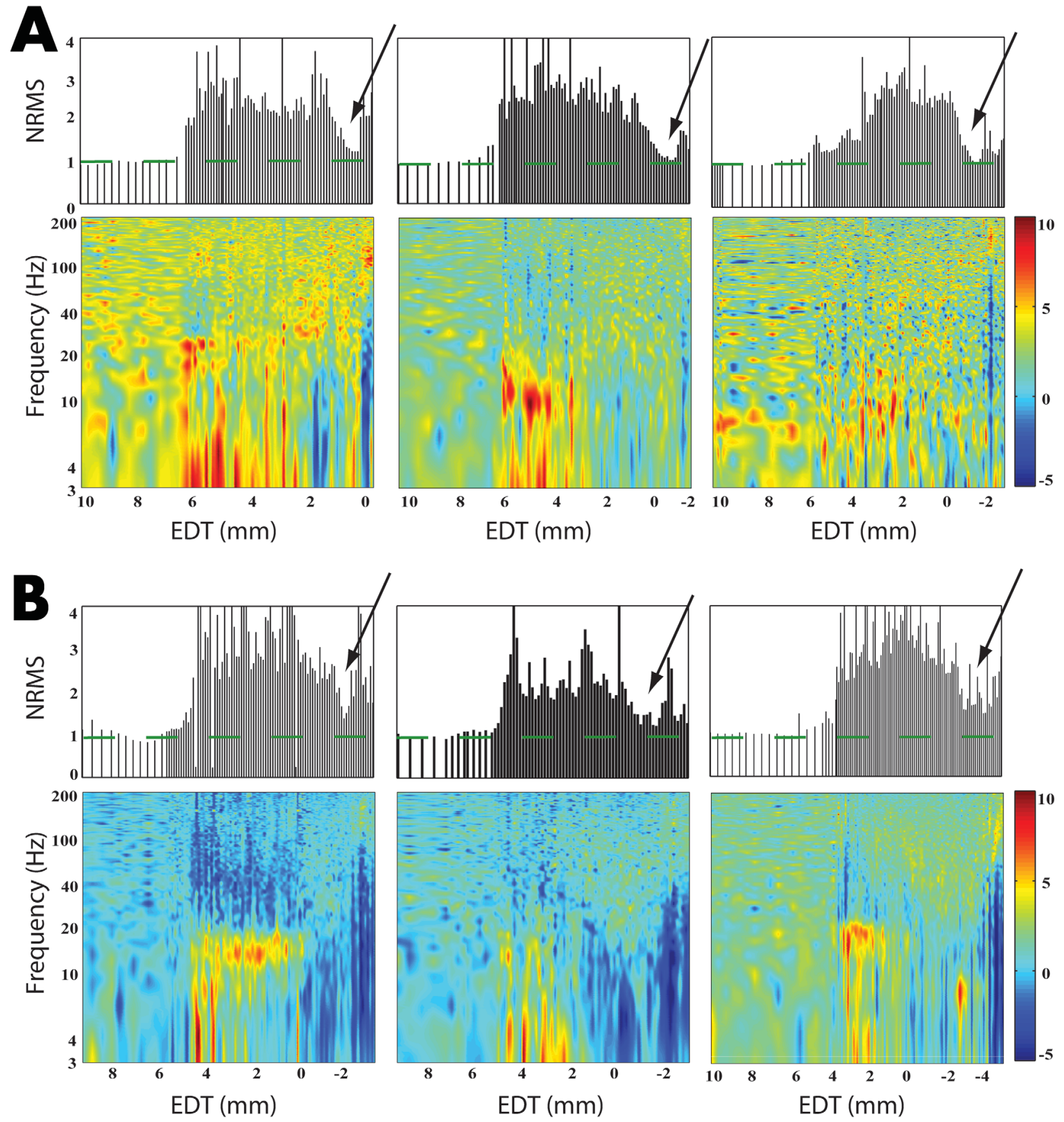

FIG. 2. STN-white matter transition versus STN-SNr transition. (A) Clearly defined STN-white matter (STN-WM) transition in three example trajectories (from three patients). The top three graphs represent the normalized root mean square (NRMS) analysis as a function of EDT. The bottom three graphs represent the power spectral distribution (PSD) spectrogram of the data, in relation to EDT on the x-axis. The arrow on each trajectory points to the transition between the STN and the WM (determined by an expert neuorophysiologist). The green dashed lines represent the baseline of the NRMS (i.e., WM). (B) The same as in A, but for the STN-SNr transition. The power spectral density color-scale represents 10 log ${ }_{10}$ (power spectral density / average power spectral density). The arrow on each trajectory points to the transition between the STN and the SNr (determined by an expert neuorophysiologist). EDT = estimated distance to target (defined as the dorsolateral STN target according to preoperative imaging).

\section{Results}

\section{Power Spectra Features Help to Discriminate STN From SNr Recordings}

The NRMS values calculated from the MERs were very effective in detecting the STN border with the white matter. As presented in the 3 examples in Figure 2A (top), the STN-entry and STN-exit borders appear as a sharp increase and decrease in the NRMS, respectively. ${ }^{12,23}$ In these "easy" cases the electrode traversed the STN and entered the $\mathrm{SNr}$ after passing through the white matter. The power spectra of these $\mathrm{SNr}$ (Fig. 2A, bottom) depict a unique signature: blue vertical lines indicating a reduction in relative power at lower frequencies. However, some trajectories lacked a clearly defined 
VALSKY E T A L $)$
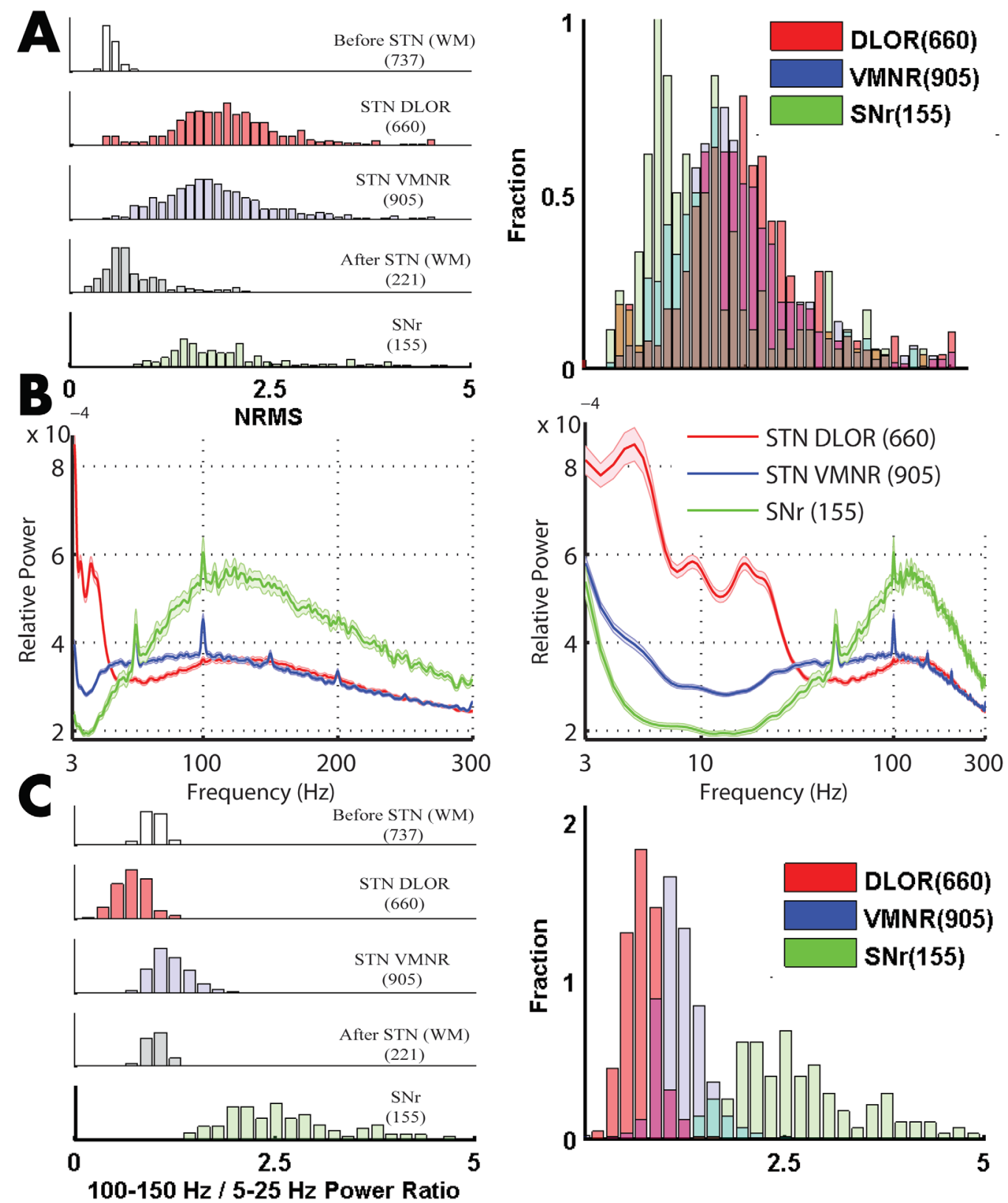

FIG. 3. "100-150 Hz / 5-25 Hz Power Ratio" separates STN from SNr better than NRMS. (A) The left hand figure illustrates the NRMS distribution for dorsal STN (red), ventral STN (blue), SNr (green), white matter before STN (white), white matter after STN (gray). Right hand figure same as left, but three subcortical structures are superimposed on the x-axis, and show the overlap in NRMS distribution of the STN and the SNr. (B) The left hand figure illustrates the power spectral density as a function of the frequency, with a linear scale plot in the DLOR STN (red), VMNR STN (blue), SNr (green). The right hand figure is the same as left, but with a logarithmic scale plot of the $\mathrm{x}$-axis. The shaded regions mark SEMs. (C) The left hand figure illustrates "100-150 Hz / 5-25 Hz Power Ratio" distribution in five regions. Right hand figure same as left, but has the three subcortical structures superimposed on the x-axes.

STN exit (eg, Fig. 2B). These are the "hard" cases in which there is no clear transient reduction in the NRMS (NRMS gap), most probably because the electrode traversed the STN and entered the SNr without passing through the white matter after the STN. Although the SNr cannot be identified by the NRMS in these cases, the $\mathrm{SNr}$ was identified by the electrophysiologist and can be seen in the power spectra (Fig. 2B, bottom), as depicted by the vertical blue lines. These examples suggest that power spectra characteristics can be used to assist in detection of the STN exit, especially for cases without an STNWM transition and NRMS gap.

To evaluate the ability of the NRMS to distinguish the STN from the SNr, we calculated the distribution of their NRMS values. Figure 3A shows the overlap in the NRMS distribution of 660 MERs in the STN DLOR, 990 MERs in the STN VMNR, and 155 MERs in the $\mathrm{SNr}$ (training data set). The significant overlap between the different distributions suggests 


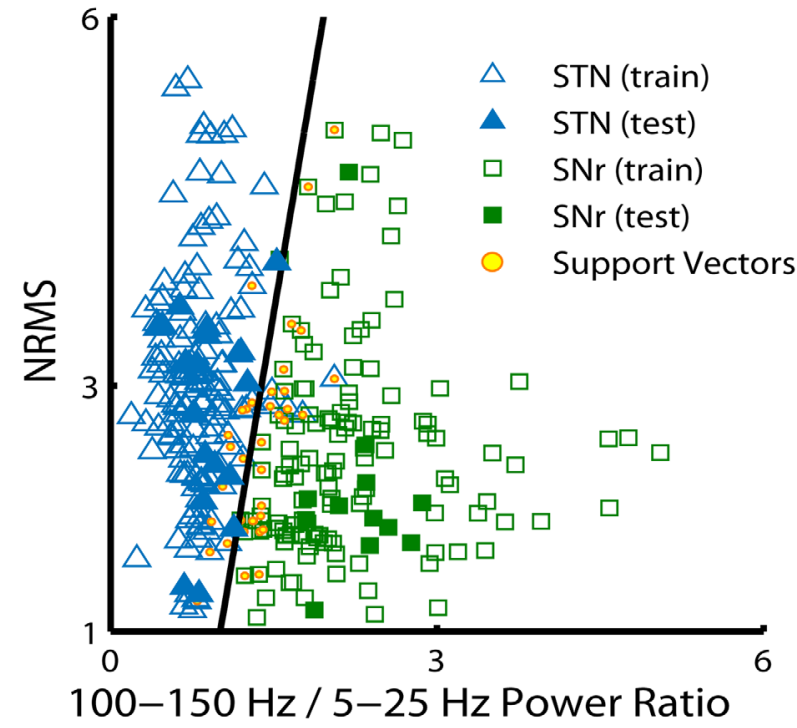

FIG. 4. A linear support vector machine classifier provides high performance discrimination between the STN and SNr populations. A support vector machine (SVM) classifier was trained and tested on 155 randomly selected samples from the STN and all 155 samples from the SNr, using NRMS and the "100-150 Hz / 5-25 Hz Power Ratio" features. The linear-kernel decision boundary is used to classify the trained data for the $\mathrm{SNr}$ (hollow square; green) and the STN (hollow triangle; blue); then new data points are classified as $\mathrm{SNr}$ (solid square; green) or STN (solid triangle; blue). Yellow circles (within the hollow squares and triangles) represent the support vectors defining the decision boundary between the STN and SNr samples.

that there is no clear separation between the STN and the $\mathrm{SNr}$ using NRMS. In contrast, Figure 3B, illustrating the mean PSD of the STN and SNr recordings, suggests that features from the PSD could be used to discriminate the STN from the SNr. In line with the characteristic signature of the STN and SNr in the spectrograms (Fig. 2), the average PSDs of the 2 STN domains and the $\mathrm{SNr}$ revealed different nonoverlapping features. The mean SNr PSD (Fig. 3B, green trace) presented decreased activity in the $5-$ to $25-\mathrm{Hz}$ band compared with the mean PSD of the STN DLOR and VMNR (Fig. 3B, red and blue traces). In addition, the mean PSD in the $\mathrm{SNr}$ displayed increased activity in the 85- to 300-Hz band (Fig. 3B, green trace).

To determine quantitatively which part of the power spectra enables the best discrimination of the STN from the $\mathrm{SNr}$, we examined 10 (approximately logarithmically distributed) bands along the frequency axis in the power spectra. The mean power in 2 frequency bands - high frequency $(100-150 \mathrm{~Hz})$ and low frequency $(5-25 \mathrm{~Hz})$ - provided the greatest discrimination between the STN and the $\mathrm{SNr}$ (discrimination matrix of 10 bands presented in Supporting Information Table S1). We therefore calculated the ratio of the power of these 2 frequency bands and termed this new feature the $100-150 \mathrm{~Hz} / 5-25 \mathrm{~Hz}$ power ratio. Figure $3 \mathrm{C}$ shows very little overlap in the distributions of $\mathrm{STN}$ and $\mathrm{SNr}$ power ratio values.

\section{Support Vector Machine Analysis Confirms the Utility of the Power Ratio for STN-SNr Discrimination}

An SVM classifier was used to examine the ability of the $100-150 \mathrm{~Hz} / 5-25 \mathrm{~Hz}$ power ratio to provide robust discrimination between the $\mathrm{SNr}$ and the STN. Figure 4 shows the result of an SVM classifier that was trained and tested on 155 randomly selected samples from the STN and all 155 samples from the SNr. A linear-kernel decision boundary was used to classify the training set as $\mathrm{SNr}$ (hollow square; green) or STN (hollow triangle; blue), and then new data points were classified as SNr (solid square; green) or STN (solid triangle; blue). Yellow circles represent the support vectors defining the decision boundary between the STN and SNr samples. Figure 4 further demonstrates the absence of correlation between NRMS and the $100-150 \mathrm{~Hz} / 5-25 \mathrm{~Hz}$ power ratio. Both these characteristics reinforce the utility of the power ratio feature as an additional attribute for classifying MERs. The discrimination performance of the SVM classifier for the entire training data set using 2 features, NRMS and $100-150 \mathrm{~Hz} / 5-25 \mathrm{~Hz}$ power ratio, was evaluated by 10 -fold cross-validation and is presented as an error matrix in Supporting Information Table S2. The overall classification accuracy rate was $97.6 \%$.

\section{Hidden Markov Model Analysis Enables Reliable Detection of STN Exit}

The hidden Markov model (HMM) procedure uses MER features and trajectory history to enable realtime decisions about electrode placement. The use of trajectory history in addition to the MER features enables the HMM procedure to "neglect" recording glitches that a classification method (eg, SVM) would classify incorrectly. Our previous HMM procedures ${ }^{23}$ did not include the $\mathrm{SNr}$ as a possible state and did not use the high frequencies $(100-150 \mathrm{~Hz})$ of the power spectrum. Here we extended the HMM procedure to discriminate between the STN and SNr using the 100$150 \mathrm{~Hz} / 5-25 \mathrm{~Hz}$ power ratio and NRMS features, together with the depth of the trajectory (ie, estimated distance to the target). The distribution of STN-exit borders was evaluated and revealed that 77 of 131 trajectories $(59 \%)$ had STN-WM transitions and that 54 of 131 trajectories $(41 \%)$ had STN-SNr transitions.

Figure 5 presents 3 examples of a typical trajectory's NRMS and PSD as well as the $100-150 \mathrm{~Hz} / 5-25 \mathrm{~Hz}$ power ratio feature. At each depth along the trajectory during the implant process the NRMS and power spectra features of the MERs are continually calculated and updated. Based on these calculations a new assessment by the HMM is made automatically in real time. An expert physiologist lowers the electrode along the trajectory until the red line appears (ie, as 

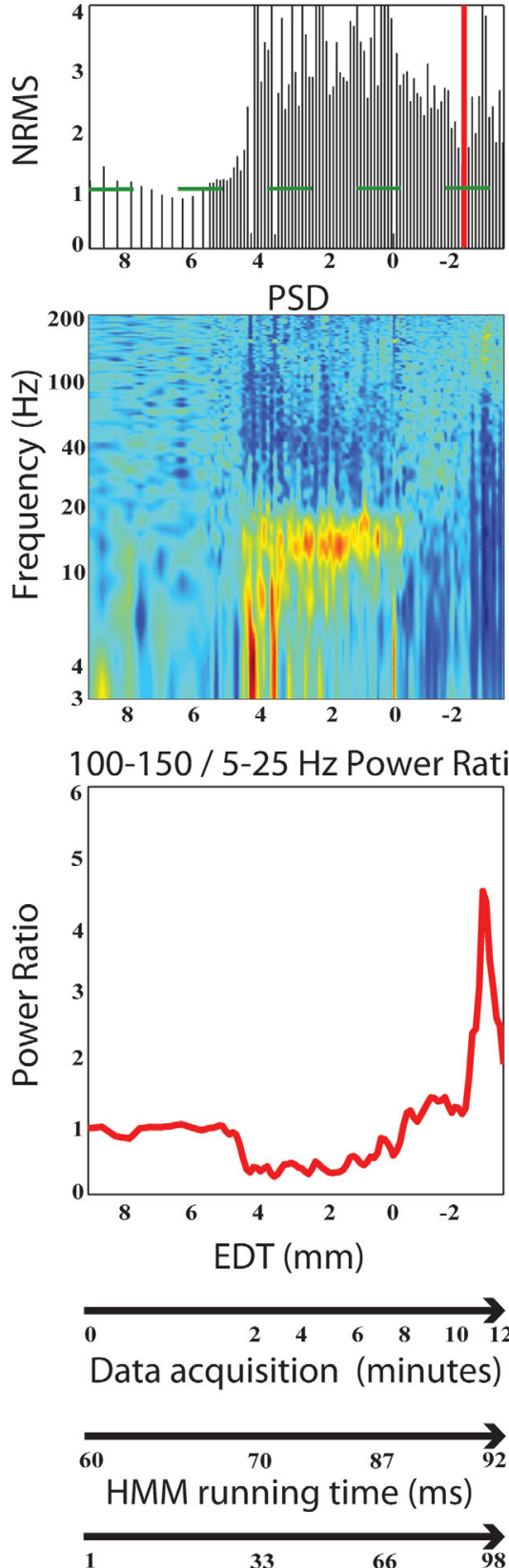

Number of MERs
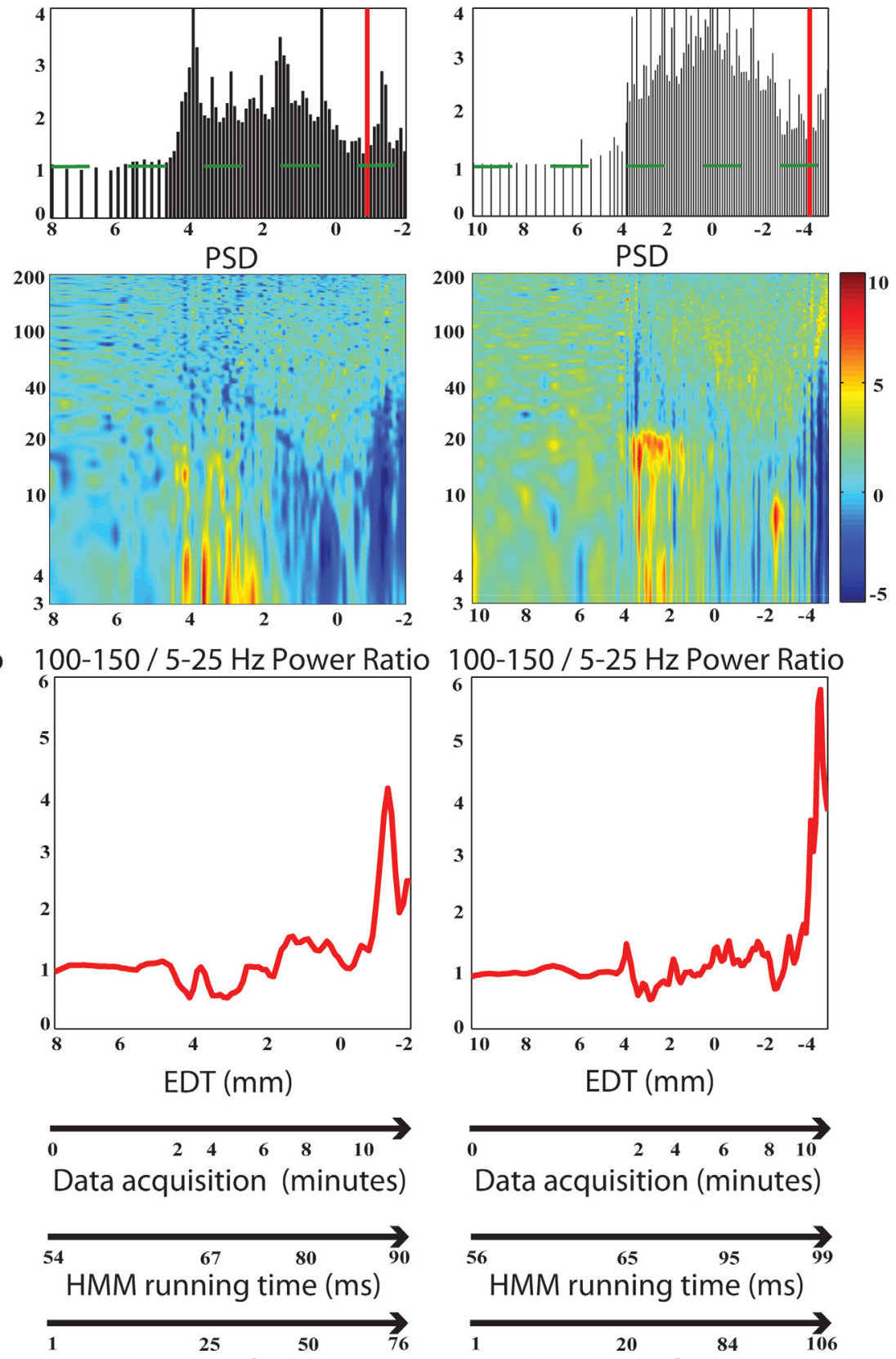

Number of MERs

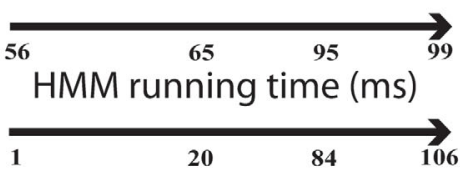

Number of MERs

FIG. 5. Robust detection of the STN-SNr transition by the "100-150 Hz / 5-25 Hz Power Ratio" feature. Top and middle panels illustrate a typical trajectory's NRMS and PSD, respectively. Bottom panel represents the "100-150 Hz / 5-25 Hz Power Ratio" feature as a function of the estimated distance to target (EDT). The three sample trajectories illustrate the direct transition from the STN to SNr. The red line in the top panel illustrates the STN-SNr transition defined by the real time HMM analysis. The dashed green lines represent the baseline of the NRMS (i.e., WM). Each segment along the trajectory was recorded for at least 4 seconds, except for a few segments where responses to active and passive limb movements were tested. The data acquisition arrow marks the real duration of a single track of the example trajectories as a function of EDT. The total recording time of a single DBS track of the example trajectories from the left to the right column: 992 seconds, 622 seconds and 608 seconds. The HMM running time arrow shows the duration of the HMM processing as a function of the number of MERs (lower arrow). MERs = micro-electrode recordings.

determined by the real-time HMM analysis). This indicates that the STN ventral border has been reached, followed by either the $\mathrm{SNr}$ or white matter. The 3 example trajectories illustrate the direct transition from the STN to the SNr. The red line in the top panel illustrates the direct STN-SNr transition defined by the real-time HMM analysis. It takes 99 milliseconds in real time to process a new trajectory and determine whether it is STN or not, as illustrated by the HMM running time in Figure 5. The time to analyze each subsequent MER is less than a millisecond per MER, making this a practical method for use during DBS surgery.

For each of the 58 trajectories in the training data set, the HMM parameters (transition and emission matrices) were estimated from the other 57 trajectories 
(leave-one-out cross-validation). The resulting mean (of all 58 trajectories) HMM transition and emission matrices are presented in Supporting Information Table S3.

The performance of the HMM was assessed with 2 measures. The first is the mean OUT location error. It is defined as the difference between the location (Human Expert's Classification), which is the location of the transition defined by the neurophysiologist, and location(HMM), which is the HMM inferred location of the transition, both measured in millimeters of estimated distance to the target. The second measure is the OUT transition error, which is defined as an OUT location error greater than $1 \mathrm{~mm}$. Hits were the number of correctly detected OUT transitions. Misses were the number of OUT transitions (according to the human expert's decision) that the HMM procedure did not detect.

The OUT location error for both STN-SNr and STN-WM demonstrated better mean and standard deviation than that found by previous methods. ${ }^{12,23}$ The performance of the OUT location error on the training data set had an error of $0.1 \pm 0.34 \mathrm{~mm}$ (mean \pm standard deviation), with 2 misses of 58 trajectories (97\% hits).

The HMM procedure has to deal with a heterogeneous variation of trajectories, as some transitions are from VMNR STN to SNr and others from white matter before STN to SNr. Because an automatic detection algorithm that can be used in the operating room needs to function on novel data without being continually adjusted, it is important to demonstrate that the HMM procedure can work with completely novel data. Therefore, 73 other trajectories (from 51 patients, all trajectories included both the STN and $\mathrm{SNr}$ ) were evaluated by the HMM procedure. The HMM procedure identified the STN exit with an error of $0.04 \pm 0.18 \mathrm{~mm}$. Using the $1-\mathrm{mm}$ threshold, the OUT transition error of the novel data set committed 4 misses of 73 trajectories ( $94 \%$ hits), which is better than that found when applying the previous HMM procedure $^{23}$ and Bayesian method ${ }^{12}$ (12 misses of 73 trajectories, $83 \%$ hits, and error $=0.50 \pm 0.59 \mathrm{~mm}$, respectively). The performance of the new HMM procedure was shown to be robust to the specified threshold because threshold values of 0.5 and $0.15 \mathrm{~mm}$ produced similar quantitative results ( 4 and 7 misses of 73 trajectories, respectively).

\section{Discussion}

We described a computational machine-learning procedure with a new feature, namely, the ratio of high-frequency $(100-150 \mathrm{~Hz})$ power to low-frequency $(5-25 \mathrm{~Hz})$ power, which enables high accuracy discrimination of the STN from the SNr. We used an SVM procedure to verify that the $100-150 \mathrm{~Hz} / 5-25 \mathrm{~Hz}$ power ratio is a reliable feature for discriminating the STN and $\mathrm{SNr}$ populations. Then, we utilized an HMM procedure using the MER features together with the trajectory history to detect the STN exit either to the white matter or SNr.

The MERs along preplanned trajectories are commonly used to confirm the STN territory during DBS surgery for Parkinson's disease; however, there is a lack of consensus on whether the MER allows for reliable separation between the STN exit point and the $\mathrm{SNr}$ entry point. Across the ventral region of the STN, there are fewer kinesthetic neurons, ${ }^{22}$ and the STN VMNR neurons are characterized by consistently reduced $\beta$-band and increased gamma $(30-100 \mathrm{~Hz})$ activity. ${ }^{13}$ Similarly, the discharge pattern of the neurons in the SNr (below the STN target) lacks the $\beta$ band and tremor-frequency oscillations, but has increased gamma activity. ${ }^{14-18}$ In addition, islands of cells have been observed with firing characteristics of both SNr and STN cells. ${ }^{22}$ Therefore, the electrophysiological determination of the transition from the STN to the $\mathrm{SNr}$ is ambiguous and difficult to evaluate.

Several studies have developed automatic detection and visualization not only for the STN, but also for the $\mathrm{SNr}$ based on objective and quantitative MER features. ${ }^{12,20,22,25-27}$ Some of these studies have used features that require spike detection algorithms to identify the firing pattern. ${ }^{25-27}$ Although these features may aid in detecting the STN ventral border near the $\mathrm{SNr}$, it is still computationally challenging to calculate neuronal spike characteristics in a real-time intraoperative scenario. ${ }^{12}$ Moreover, the ideal isolation of single units requires 5 - to $10-\mu \mathrm{m}$ steps of electrodes and is very time consuming. In contrast, NRMS values that are based on unsorted multiunit activity are easy to measure. The STN entry and STN exit often manifest as a sharp increase and decrease in the NRMS, respectively. ${ }^{12,23}$ Some studies have used the NRMS together with spectral features of the analog signal, which are computationally efficient to calculate. ${ }^{20,22,27}$ However, these spectral features do not lead to reliable or robust identification of the transition between the STN and the SNr. Here, we divided the 5- to $300-\mathrm{Hz}$ range power spectra into frequency bands. This division allowed us to determine which frequency bands contained the largest difference between the STN and the $\mathrm{SNr}$ and to accurately detect the STN ventral border.

When implementing the NRMS and features from the power spectra, there are several ways to differentiate the STN from the SNr using automatic detection methods. Some studies have proposed rule-based detection methods ${ }^{22,27}$; however, they are unable to detect the direct STN-SNr transitions. For example, despite that Cagnan et $\mathrm{al}^{27}$ used the power spectra of tremor and the alpha band $(3-12 \mathrm{~Hz})$, beta band $(13-30 \mathrm{~Hz})$, and 
gamma band $(31-100 \mathrm{~Hz})$ as features, their algorithm still required a white matter gap in the trajectory between the STN and the SNr to detect the SNr. Furthermore, rule-based detection systems tend to be overly complex and may not generalize to other surgical centers. Other studies ${ }^{12,23,25}$ have used machine-learning techniques to automatically extract the "rules" or decision boundaries to discriminate between the STN and the SNr. These machine-learning procedures are either unsupervised and involve extracting patterns using unlabeled training data (that still require labeling of the output $)^{25}$ or supervised, which require the labeling of the electrophysiological signals used for training. ${ }^{12,23}$ For example, Wong et $\mathrm{al}^{25}$ used a clustering algorithm (unsupervised machine learning) that returns a prespecified number of clusters, but then requires the human observer to label the clusters. The main drawback of these techniques is that they do not take the previous location of the MERs into account when determining the electrode's current location. A supervised technique that uses the electrode's location is HMM. For example, Zaidel et $\mathrm{al}^{23}$ combined the power spectra of $\beta$-band features and NRMS to locate the STN and its subterritories. The 2 advantages of the HMM procedure are the short recording time needed for location analysis and low computational cost. Our approach goes beyond this previous work by delineating the borders between STN and SNr which thus enables intraoperative application with greater accuracy.

Accurate discrimination between the STN and the $\mathrm{SNr}$ is of crucial importance for achieving optimal therapeutic benefits while avoiding psychiatric complications for PD DBS procedures. The beneficial effects of bilateral STN DBS on motor symptoms and quality of life have been repeatedly confirmed in patients with advanced $\mathrm{PD}^{28}$; however, psychiatric complications induced by STN DBS have also been reported. ${ }^{29,30}$ In some patients with PD with impulse control disorders, their abnormal behavior may be provoked by stimulation with a ventral contact of the DBS lead and suppressed by switching off this contact. ${ }^{6-9}$ It also has been reported that manic ${ }^{31,32}$ and depressive ${ }^{5}$ symptoms are induced by stimulation of active contacts located in the SNr. On the other hand, the $\mathrm{SNr}$ is thought to be particularly involved in balance control during gait. ${ }^{33}$ The combined stimulation of the $\mathrm{SNr}$ and the STN has been reported to improve axial symptoms (including freezing of gait, balance, and posture) compared with standard STN stimulation. ${ }^{10,11}$ In summary, automatic and reliable localization of the direct STN$\mathrm{SNr}$ transition and STN lower-border detection could lead to improved localization of DBS leads and better DBS clinical outcomes. It takes 99 milliseconds in real time to process a new trajectory and decide whether it is STN or not, making this a practical method for use during DBS surgery. This analysis tool can be easily learned and employed in the DBS operating room. Future studies might incorporate MER data from multiple centers to test the applicability of these algorithms for automatic navigation in DBS surgery.

\section{References}

1. Benabid AL, Chabardes S, Mitrofanis J, Pollak P. Deep brain stimulation of the subthalamic nucleus for the treatment of Parkinson's disease. Lancet Neurol 2009;8(1):67-81.

2. Krack P, Batir A, Van Blercom N, et al. Five-year follow-up of bilateral stimulation of the subthalamic nucleus in advanced Parkinson's disease. N Engl J Med 2003;349(20):1925-1934.

3. Limousin P, Pollak P, Benazzouz A, et al. Bilateral subthalamic nucleus stimulation for severe Parkinson's disease. Mov Disord 1995;10(5):672-674.

4. Machado A, Rezai AR, Kopell BH, Gross RE, Sharan AD, Benabid A-L. Deep brain stimulation for Parkinson's disease: surgical technique and perioperative management. Mov Disord 2006;21(Suppl 1):S247-S258.

5. Bejiani B-P, Damier P, Arnulf I, et al. Transient acute depression induced by high-frequency deep-brain stimulation. $N$ Engl J Med 1999;340(19):1476-1480.

6. Raucher-Chéné D, Charrel C-L, de Maindreville AD, Limosin F. Manic episode with psychotic symptoms in a patient with Parkinson's disease treated by subthalamic nucleus stimulation: improvement on switching the target. J Neurol Sci 2008;273(1-2):116-117.

7. Mallet L, Schüpbach M, N'Diaye K, et al. Stimulation of subterritories of the subthalamic nucleus reveals its role in the integration of the emotional and motor aspects of behavior. Proc Natl Acad Sci U S A 2007;104(25):10661-10666.

8. Mandat TS, Hurwitz T, Honey CR. Hypomania as an adverse effect of subthalamic nucleus stimulation: report of two cases. Acta Neurochir (Wien) 2006;148(8):895-897; discussion 898.

9. Kulisevsky J, Berthier ML, Gironell A, Pascual-Sedano B, Molet J, Parés P. Mania following deep brain stimulation for Parkinson's disease. Neurology 2002;59(9):1421-1424.

10. Weiss D, Walach M, Meisner C, et al. Nigral stimulation for resistant axial motor impairment in Parkinson's disease? A randomized controlled trial. Brain 2013;136(7):2098-2108.

11. Weiss D, Breit S, Wächter T, Plewnia C, Gharabaghi A, Krüger R. Combined stimulation of the substantia nigra pars reticulata and the subthalamic nucleus is effective in hypokinetic gait disturbance in Parkinson's disease. J Neurol 2011;258(6):1183-1185.

12. Moran A, Bar-Gad I, Bergman H, Israel Z. Real-time refinement of subthalamic nucleus targeting using Bayesian decision-making on the root mean square measure. Mov Disord 2006;21(9):1425-1431.

13. Zaidel A, Spivak A, Grieb B, Bergman H, Israel Z. Subthalamic span of $\beta$ oscillations predicts deep brain stimulation efficacy for patients with Parkinson's disease. Brain 2010;133(7):2007-2021.

14. Sterio D, Zonenshayn M, Mogilner AY, et al. Neurophysiological refinement of subthalamic nucleus targeting. Neurosurgery 2002; 50(1):58-67; discussion 67-69.

15. Bejjani B-P, Dormont D, Pidoux B, et al. Bilateral subthalamic stimulation for Parkinson's disease by using three-dimensional stereotactic magnetic resonance imaging and electrophysiological guidance. J Neurosurg 2000;92(4):615-625.

16. Rodriguez-Oroz MC, Rodriguez M, Guridi J, et al. The subthalamic nucleus in Parkinson's disease: somatotopic organization and physiological characteristics. Brain 2001;124(Pt 9):1777-1790.

17. Benazzouz A, Breit S, Koudsie A, Pollak P, Krack P, Benabid A-L. Intraoperative microrecordings of the subthalamic nucleus in Parkinson's disease. Mov Disord 2002;17(Suppl 3):S145-S149.

18. Hutchison WD, Allan RJ, Opitz H, et al. Neurophysiological identification of the subthalamic nucleus in surgery for Parkinson's disease. Ann Neurol 1998;44(4):622-628.

19. McEvoy J, Ughratdar I, Schwarz S, Basu S. Electrophysiological validation of STN-SNr boundary depicted by susceptibilityweighted MRI. Acta Neurochir (Wien) 2015;157(12):2129-2134.

20. Falkenberg JH, McNames J, Favre J, Burchiel KJ. Automatic analysis and visualization of microelectrode recording trajectories to 
the subthalamic nucleus: preliminary results. Stereotact Funct Neurosurg 2006;84(1):35-44; discussion 44-45.

21. Danish SF, Baltuch GH, Jaggi JL, Wong S. Determination of subthalamic nucleus location by quantitative analysis of despiked background neural activity from microelectrode recordings obtained during deep brain stimulation surgery. J Clin Neurophysiol 2008;25(2):98-103.

22. Novak P, Daniluk S, Ellias S a, Nazzaro JM. Detection of the subthalamic nucleus in microelectrographic recordings in Parkinson disease using the high-frequency $(>500 \mathrm{hz})$ neuronal background. Technical note. J Neurosurg 2007;106(1):175-179.

23. Zaidel A, Spivak A, Shpigelman L, Bergman H, Israel Z. Delimiting subterritories of the human subthalamic nucleus by means of microelectrode recordings and a hidden Markov model. Mov Disord 2009;24(12):1785-1793.

24. Taghva A. Hidden semi-Markov models in the computerized decoding of microelectrode recording data for deep brain stimulator placement. World Neurosurg 2011;75(5-6):758-763.e4.

25. Wong S, Baltuch GH, Jaggi JL, Danish SF. Functional localization and visualization of the subthalamic nucleus from microelectrode recordings acquired during DBS surgery with unsupervised machine learning. J Neural Eng 2009;6(2):026006.

26. Pinzon-Morales RD, Orozco-Gutierrez AA, CastellanosDominguez G. Novel signal-dependent filter bank method for identification of multiple basal ganglia nuclei in Parkinsonian patients. J Neural Eng 2011;8(3):036026.

27. Cagnan H, Dolan K, He X, et al. Automatic subthalamic nucleus detection from microelectrode recordings based on noise level and neuronal activity. J Neural Eng 2011;8(4):046006.
28. Deuschl G, Schade-Brittinger C, Krack P, et al. A randomized tria of deep-brain stimulation for Parkinson's disease. N Engl J Med 2006;355(9):896-908.

29. Voon V, Kubu C, Krack P, Houeto J-L, Tröster AI. Deep brain stimulation: neuropsychological and neuropsychiatric issues. Mov Disord 2006;21(Suppl 1):S305-S327.

30. Saint-Cyr JA, Trépanier LL, Kumar R, Lozano AM, Lang AE. Neuropsychological consequences of chronic bilateral stimulation of the subthalamic nucleus in Parkinson's disease. Brain 2000; 123(Pt 1):2091-2108.

31. Ulla M, Thobois S, Lemaire J-J, et al. Manic behaviour induced by deep-brain stimulation in Parkinson's disease: evidence of substantia nigra implication? J Neurol Neurosurg Psychiatry 2006;77(12): 1363-1366.

32. Ulla M, Thobois S, Llorca P-M, et al. Contact dependent reproducible hypomania induced by deep brain stimulation in Parkinson's disease: clinical, anatomical and functional imaging study. J Neurol Neurosurg Psychiatry 2011;82(6):607-614.

33. Chastan N, Westby GWM, Yelnik J, et al. Effects of nigral stimulation on locomotion and postural stability in patients with Parkinson's disease. Brain 2009;132(1):172-184

\section{Supporting Data}

Additional Supporting Information may be found in the online version of this article at the publisher's web-site. 
PhD Thesis, Dan Valsky

II. Designing a real-time striato-pallidal border detection method

a. Valsky, D., Blackwell, K. T., Tamir, I., Eitan, R., Bergman, H., \& Israel, Z. (2020). Real-time machine learning classification of pallidal borders during deep brain stimulation surgery. Journal of neural engineering, 17(1), 016021. https://doi.org/10.1088/1741-2552/ab53ac 
PAPER

\section{Real-time machine learning classification of pallidal borders during deep brain stimulation surgery}

\section{Recent citations}

What is the true discharge rate and pattern of the striatal projection neurons in Parkinson's disease and Dystonia? Dan Valsky et al

To cite this article: Dan Valsky et al 2020 J. Neural Eng. 17016021

View the article online for updates and enhancements.

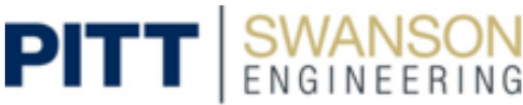

B I O

CLICK HERE FOR FURTHER DETAILS
The Department of Bioengineering at the University of Pittsburgh Swanson School of Engineering invites applications from accomplished individuals with a $\mathrm{PhD}$ or equivalent degree in bioengineering, biomedical engineering, or closely related disciplines for an open-rank, tenured/tenure-stream faculty position. We wish to recruit an individual with strong research accomplishments in Translational Bioengineering (i.e., leveraging basic science and engineering knowledge to develop innovative, translatable solutions impacting clinical practice and healthcare), with preference given to research focus on neuro-technologies, imaging, cardiovascular devices, and biomimetic and biorobotic design. It is expected that this individual will complement our current strengths in biomechanics, bioimaging, molecular, cellular, and systems engineering, medical product engineering, neural engineering, and tissue engineering and regenerative medicine. In addition, candidates must be committed to contributing to high quality education of a diverse student body at both the undergraduate and graduate levels. 


\title{
Journal of Neural Engineering
}

\section{PAPER}

\section{Real-time machine learning classification of pallidal borders during deep brain stimulation surgery}

RECEIVED

29 October 2018

REVISED

30 October 2019

ACCEPTED FOR PUBLICATION

1 November 2019

PUBLISHED

6 January 2020

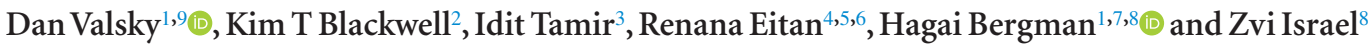 \\ 1 The Edmond and Lily Safra Center for Brain Research (ELSC), The Hebrew University, Jerusalem, Israel \\ 2 Department of Bioengineering, George Mason University, Fairfax, VA, United States of America \\ 3 Department of Neurosurgery, Rabin Medical Center, Petach Tikva, Israel \\ 4 Faculty of Medicine, Department of Medical Neurobiology, Hebrew University of Jerusalem \\ Jerusalem Mental Health Center, Hebrew University Medical School, Jerusalem, Israel \\ 6 Functional Neuroimaging Laboratory, Department of Psychiatry, Brigham and Women's Hospital, Harvard Medical School, Boston, \\ MA, United States of America \\ 7 Department of Medical Neurobiology (Physiology), Institute of Medical Research-Israel-Canada (IMRIC), The Hebrew University- \\ Hadassah Medical School, Jerusalem, Israel \\ 8 Department of Neurosurgery, Center for Functional and Restorative Neurosurgery, Hadassah-Hebrew University Medical Center, \\ Jerusalem, Israel \\ 9 Author to whom any correspondence should be addressed. \\ E-mail:dan.valsky@mail.huji.ac.il
}

Keywords: Deep brain stimulation, Parkinson's disease, machine learning, striatum, dystonia, pallidum, light general anesthesia

Supplementary material for this article is available online

\begin{abstract}
Objective. Deep brain stimulation (DBS) of the internal segment of the globus pallidus (GPi) in patients with Parkinson's disease and dystonia improves motor symptoms and quality of life. Traditionally, pallidal borders have been demarcated by electrophysiological microelectrode recordings (MERs) during DBS surgery. However, detection of pallidal borders can be challenging due to the variability of the firing characteristics of neurons encountered along the trajectory. MER can also be time-consuming and therefore costly. Here we show the feasibility of real-time machine learning classification of striato-pallidal borders to assist neurosurgeons during DBS surgery. Approach. An electrophysiological dataset from 116 trajectories of 42 patients consisting of 11774 MER segments of background spiking activity in five classes of disease was used to train the classification algorithm. The five classes included awake Parkinson's disease patients, as well as awake and lightly anesthetized genetic and non-genetic dystonia patients. A machine learning algorithm was designed to provide prediction of the striato-pallidal borders, based on hidden Markov models (HMMs) and the $\mathrm{L}_{1}$-distance measure in normalized root mean square (NRMS) and power spectra of the MER. We tested its performance prospectively against the judgment of three electrophysiologists in the operating rooms of three hospitals using newly collected data. Main results. The awake and the light anesthesia dystonia classes could be merged. Using MER NRMS and spectra, the machine learning algorithm was on par with the performance of the three electrophysiologists across the striatum-GPe, GPe-GPi, and GPi-exit transitions for all disease classes. Significance. Machine learning algorithms enable real-time GPi navigation systems to potentially shorten the duration of electrophysiological mapping of pallidal borders, while ensuring correct pallidal border detection.
\end{abstract}

\section{Introduction}

Deep brain stimulation (DBS) of the subthalamic nucleus (STN) and the internal segment of the globus pallidus (GPi) is the most common and effective surgical procedure for advanced Parkinson's disease (PD) [1-4] and dystonia [5-9], with over 150000 patients treated worldwide [10]. Implanting the DBS lead in the optimal location is critical for optimal outcome. Recent data from North American databases suggest that in intracranial neurostimulation therapies up to $48.5 \%$ of revisions are due to suboptimally placed electrodes $[11,12]$. Many studies of human intraoperative localization of DBS targets have focused 
on the STN alone $[13,14]$. However, there is increasing interest in identifying pallidal physiomarkers [15-17]. The GPi is the primary target for dystonia, and it is increasingly used for $\mathrm{PD}$, based on the finding of equivalent motor benefits in three out of four randomized trials of STN versus GPi-DBS [1-4]. Two recent studies have reported that an inappropriate lead placement is the most common cause for the observed therapeutic failures of GPi-DBS in dystonia patients $[18,19]$.

Successful implantation of the DBS lead within the GPi may be facilitated by microelectrode recording (MER), which affords accurate spatial demarcation of the external segment of the globus pallidus (GPe) and GPi borders [20]. However, MER can be timeconsuming and therefore costly. Here, we describe a computational method that enables neurosurgeons to actively track the delineation of the striato-GPeGPi transitions along pre-planned trajectories. Recent work in pallidal physiological mapping [15-17] has not included intraoperative computer-aided classification of the striato-pallidal borders, or has not compared the spatial oscillatory distribution of the neuronal activity within striatum, GPe and GPi across different classes of the disease in awake and lightly anesthesized patients.

Several factors can make electrophysiological determination of the striato-pallidal borders challenging, in particular an uninterrupted GPe-GPi transition [21], since there is no decrease in neural activity. In addition, the cells in the striatum may have transient episodes of high-frequency discharge (due to injury potentials, provoked by movements of the microelectrode) resembling GPe cells [22-24]. Finally, in patients under light anesthesia there may be a significant suppression of both spontaneous and evoked neuronal discharges from pallidal neurons $[25,26]$.

Although recent imaging protocols have been able to improve GPi targeting [27], many experts still consider that electrophysiology is necessary to identify and verify target borders intraoperatively [28]. This is due to the inherent resolution limitations of neuroimaging and co-registration techniques, and possible brain shifts during DBS surgery [29-31]. Shorter surgery times can also help limit the cerebrospinal fluid leak [32], and therefore brain shifts [29-31]. Because the method described here does not require spike detection algorithms or electrophysiology expertise to perform and interpret the findings, the duration of the electrophysiological mapping of the target is likely to be significantly shortened.

\section{Methods}

\section{Patients}

Patients with PD and isolated dystonia were recruited from the movement disorders clinics at the Hadassah Medical Center in Jerusalem. All patients were scheduled to undergo implantation of DBS electrodes into the GPi and underwent MR imaging, and evaluation for motor and non-motor impairment within the $30 \mathrm{~d}$ prior to surgery. We recorded the neuronal extracellular activity from 32 patients with dystonia and ten patients with PD. In 13 patients with dystonia, recordings were performed in the awake state, without any sedation or anesthesia. In the other 19 dystonic patients, recordings were performed under light general anesthesia. Recordings in all PD patients were performed in the awake state, at least $12 \mathrm{~h}$ following withdrawal of dopaminergic medication. Ten additional patients were used to test the robustness of the algorithm, as explained in Validation. Patient demographic information appears in supplementary table S1 (stacks.iop.org/JNE/17/016021/mmedia). Written informed consent was obtained from all patients and the study was approved by the Institutional Review Board of Hadassah Hospital in accordance with the Helsinki Declaration (reference code: 0168-10-HMO).

\section{Anesthesia}

General anesthesia was induced with intravenous propofol (1.5-2 mg kg-1) and alfentanil (10 $\mu \mathrm{g}$ $\left.\mathrm{kg}^{-1}\right)$. Neuromuscular blockade was achieved with vecuronium $\left(0.1 \mathrm{mg} \mathrm{kg}^{-1}\right)$ to facilitate endotracheal intubation. Anesthesia was then maintained with nitrous oxide in oxygen (70\%:30\%) and isoflurane (prior to 2016, 0.9\%-1.2\%) or sevoflurane (from 2016; $0.9 \%-2 \%)$. Before fixing the frame and the skin incision, local anesthetic (0.5\% bupivacaine, $2 \%$ lidocaine 50:50) was injected. Pallidal spiking activity is highly dependent on the level of anesthesia. The deeper the anesthesia, the less spiking activity can be detected in the pallidum $[25,26]$. To achieve light anesthesia, at least $10 \mathrm{~min}$ prior to MER, the nitrous oxide, isoflurane and sevoflurane dose were reduced to the point where single neuronal units could be observed (in the range of 0.1-0.3 minimum alveolar concentration (MAC) of nitrous oxide plus $0.2-0.3$ MAC of isoflurane or sevoflurane; total MAC achieved 0.3-0.6 MAC). This level was not further adjusted during the course of MER, and was similar across all patients (with dystonia) operated under light general anesthesia included in this study. During the light anesthesia, neuromuscular blockade was continued, ambient light and noise in the operating room were reduced, and the heart rate and blood pressure were closely monitored. Subsequent to MER, anesthesia was restored to the range prior to MER. For surgeries that were performed without general anesthesia, the patients' fully aroused state was continuously confirmed by a team member.

\section{Data acquisition}

The data were acquired using two systems: MicroGuide (prior to 2015, previously described [33]) and Neuro Omega (from 2015, Alpha Omega, Nazareth, Israel). In the Neuro Omega system, raw and spiking signals were 
amplified by 20 , and sampled at $44 \mathrm{kHz}$ by a 16-bit $\mathrm{A} / \mathrm{D}$ converter (using $\pm 1.25 \mathrm{~V}$ input range; i.e. $\sim 2 \mu \mathrm{V}$ amplitude resolution). The raw signal was bandpass filtered from 0.075 to $10 \mathrm{k} \mathrm{Hz}$, using a hardware 2 and 3 poles Butterworth filter, respectively. The spiking signal was bandpass filtered from 300 to $9000 \mathrm{~Hz}$, using a hardware 4-poles Butterworth filter.

\section{Microelectrode recordings (MERs)}

For each hemisphere and for every recording session, an exploratory MER using one or two microelectrode trajectories ( $2 \mathrm{~mm}$ apart) was determined starting at $15 \mathrm{~mm}$ above the image-based calculated target. Our trajectories followed a double-oblique approach through the striatum and the GPe towards the ventral border of the posterior-lateral portion of the GPi target. The electrophysiological characteristics of these neighboring structures have been published elsewhere [22-24] and were used in the operating room to manually tag the borders of the structures. Some of these recordings were made by a single microelectrode trajectory (instead of two) to accommodate for cortical anatomy under the burr hole and brain blood vessels. The trajectory recordings were not continuous, but in steps as detailed below.

A total of 84 exploratory MERs (from one or two microelectrodes on each recording) were analyzed, yielding a total of 116 microelectrode trajectories aiming at the GPi. The analysis was based on portions of data recorded at specific points (i.e. different spatial locations) along the trajectory with the electrode stationary. We term these data MER segments because they are not totally independent from each other in the sense that in each microelectrode trajectory the data were recorded from the same electrode (same impedance) and the same hemisphere. We recorded a total of 11774 MER segments within the Str, GPe and GPi.

MER segments were regularly sampled in space. For all our GPi-DBS surgeries, the depth steps of the recorded signals ranged from $200 \mu \mathrm{m}$ to $100 \mu \mathrm{m}$ and were controlled by the neurophysiologist to achieve optimal identification of the pallidal borders. Shorter steps $(\sim 100 \mu \mathrm{m})$ were used when the electrode was advanced closer to and within the presumed location of the GPi. At each step, MER segments were recorded for at least $4 \mathrm{~s}$, after the $2 \mathrm{~s}$ signal stabilization period. All MER segments were subjected to stability analysis using a custom-made algorithm and only those meeting these stability criteria were included [34]. A few MER segments were recorded for longer durations than others because the responses to active and passive limb movement were tested. The length of the MER segments were not homogenous and varied between 4 and $90 \mathrm{~s}$. We excluded MER segments when active and passive limb movements were tested in the learning phase.

\section{Neural datasets}

Two independent datasets were prepared for the training and validation of the machine learning models. The training dataset was composed of 116 microelectrode trajectories that passed through the striatum, GPe and GPi of 42 PD and dystonia patients undergoing bilateral GPi-DBS implantation. The training dataset of 116 trajectories was also used to find the optimal parameters for the hidden Markov model (HMM).

The testing dataset was composed of 32 trajectories, in ten patients from three medical centers (Hadassah-Hebrew University Medical Center $n=5$, Sourasky Medical Center $n=3$, Rabin Medical Center $n=2$ ) and was used to test the robustness of the algorithm. Subsequently, five trajectories were excluded because single neuronal units were not observed at 0.4-0.6 of MAC. Thus, 27 trajectories were included in the independent test dataset (6 Parkinson's disease patients, two genetic dystonia and two non-genetic dystonia patients). Additional details on the neuronal and MER dataset appear in the supplementary material and table S2 and S3.

\section{Root mean square (RMS)}

The RMS estimate was calculated from the multiunit activity recorded by the microelectrode at each electrode depth. During the surgery we did not attempt to optimize the signal-to-noise ratio or the isolation of single units. RMS values are susceptible to electrode properties (e.g. electrode impedance) and other artifacts related to the operating room [34]. To enable comparisons of the RMS values between trajectories and patients we normalized the RMS values. Each session's RMS in a trajectory was divided by the mean RMS of the first ten stable sessions in the same trajectory (pre-pallidum baseline RMS). This normalized RMS (NRMS) was found to be an effective measure as it reflects the relative change in the total power of the signal, which rises dramatically entering the pallidum $[35,36]$. For example, entry and exit from the GPe and GPi are marked primarily by a dramatic increase and decrease in NRMS (figure 6, top panels), respectively. Additional details are presented in the supplementary material.

\section{Power spectral density (PSD)}

The PSD was calculated from the mean-subtracted absolute value of the analog (multi-unit activity) signal $[37,38]$. The mean-subtracted signal was used to expose the frequency band of interest (below 300 $\mathrm{Hz}$ ) since the original analog data were bandpass filtered at $300-9000 \mathrm{~Hz}$ (the $300 \mathrm{~Hz}$ cutoff was used to exclude the effects of field potentials and because of operating room constraints). The average power spectral density was calculated for each trace using Welch's method, with a $3 \mathrm{~s}$ Hamming window $(50 \%$ overlap) and a spectral resolution of $1 / 3 \mathrm{~Hz}$ (sampling frequency $44 \mathrm{kHz}$, number of discrete Fourier transform points $=132000$ ). To normalize the PSD we divided the PSD by the total power of the signal from 0 to $22 \mathrm{KHz}$ (excluding PSD values within 2 
$\mathrm{Hz}$ of the $50 \mathrm{~Hz}$ power supply artifacts and their 100 and $150 \mathrm{~Hz}$ harmonics) creating a relative PSD [34]. This normalization revealed the relative power across frequencies and minimized the overlap between the measures of the NRMS and the PSD. Additional details are presented in the supplementary material.

\section{The distance in power spectral density and RMS}

The distances between regions and classes were calculated as the L1-distance (sometimes referred to as the 'Manhattan distance' - the sum of absolute differences) of the mean PSD and NRMS. Additional details are presented in the supplementary material.

\section{The HMM}

HMMs are advantageous for general and highly variable tasks across many sequence analysis classifications [39, 40]. The HMM takes the set of features extracted from the raw data as input and provides the output clustering in real-time. In previous reports $[14,41]$, the HMM procedure has successfully been used to discriminate the STN from the white matter or substantia nigra pars reticulata. This study extends our previous work by designing an online HMM algorithm that can detect the striatopallidal borders (i.e. estimate the inference states of the electrode at each depth along the trajectory). Figure 1 shows the machine learning working system.

\section{Estimating the HMM}

For purposes of the HMM procedure, a trajectory was defined as a sequence of observations, such that at any depth along the trajectory the electrode exists in one of the inference states (figure $1(\mathrm{E})$ ).
1. Str (striatum)
2. GPe
3. GPi
4. Exit (i.e. white matter)

For each trajectory, an expert electrophysiologist lowered the electrode and labelled the states. Since the recordings started at the level of the striatum, the subsequent labels were: the detection of the GPe, the demarcation between the GPe and GPi, and the detection of the GPi ventral border (GPi-exit). For the training dataset, a single neurophysiologist labelled the trajectory state for all trajectories. Detection of the striatum-GPe and GPi-exit transitions were based on intraoperative neuronal analysis by the electrophysiologist as well as the NRMS plots. The GPe-GPi transition was distinguished by visual inspection of the NRMS and PSD, by identification of an NRMS gap (white matter) and a change in oscillatory activity. Occasionally, typical border cell activity was detected at the transition and supported the decision. However, not all trajectories had clearly defined Str-GPe and GPe-GPi transitions; therefore, only trajectories that started $15 \mathrm{~mm}$ above the calculated target and traversed $>7 \mathrm{~mm}$ of GPe and GPi were included in this study. The known state transitions for different classes of disease are depicted in the NRMS plots by red lines and in the PSD plots by black lines (figure 6). These transitions defined a known state sequence for estimating and testing the HMM.

In our own practice we do not advance the microelectrode beyond the ventral border of the GPi. We are very cautious because damage to the optic tract may cause hemianopsia and stimulation that spreads to the optic tract may cause patients to experience visual perceptions commonly referred to as 'flashes' [42]. In trajectories with $>5 \mathrm{~mm}$ of GPe and $>5 \mathrm{~mm}$ within GPi we pull back the microelectrode immediately after we reach $0 \mathrm{~mm}$ or as soon as we notice a clear decline in the NRMS value. Therefore, in some trajectories the ventral border of GPi (i.e. GPi-exit) has not been reached across all classes of disease; specifically for Parkinson's disease, non-genetic and genetic dystonia patients (number of detected GPi-exit borders: $n=23,30$ and 31 out of 33,47 and 36 trajectories, respectively).

The maximum likelihood estimate of the HMM transition and emission probability matrices were estimated based on the known (human expert defined) state sequences. Since the training data were fully labelled (there were known state sequences for the whole dataset), there was no need to implement the expectation-maximization (EM) algorithm or iterative procedures (which would require initial guessing of the probability matrices), and the matrices could be directly estimated. In our HMM, transitions between states take place according to (1) a fixed probability (i.e. transition matrix and emission matrix) depending solely on the inference state of the electrode at the depth immediately preceding the current inference state and (2) a probability value calculated from the current MER.

In the operating room the MERs are considered temporal data in the spatial domain rather than time domain. We used a left-right HMM procedure for the characterization of temporal data recorded during the electrophysiological mapping of sequential brain structures (figure 2); i.e. as a sequence progresses, it is possible to remain in the same state, but it is not possible to return to a previous state (e.g. from the GPi to the GPe).

\section{Validation}

The performance of the HMM was assessed by two measures on three classification cases: striatumGPe, GPe-GPi, and GPi-exit transitions. First, we validated the machine learning algorithm using leave-one-trajectory-out cross-validation. This ensured independence between the trajectories used for developing the HMM parameters (i.e. transition and emission matrices) and the trajectories used for validation. Three HMMs were estimated for three classes of disease using the known state sequences of all trajectories, excluding one (e.g. Parkinson's disease, 

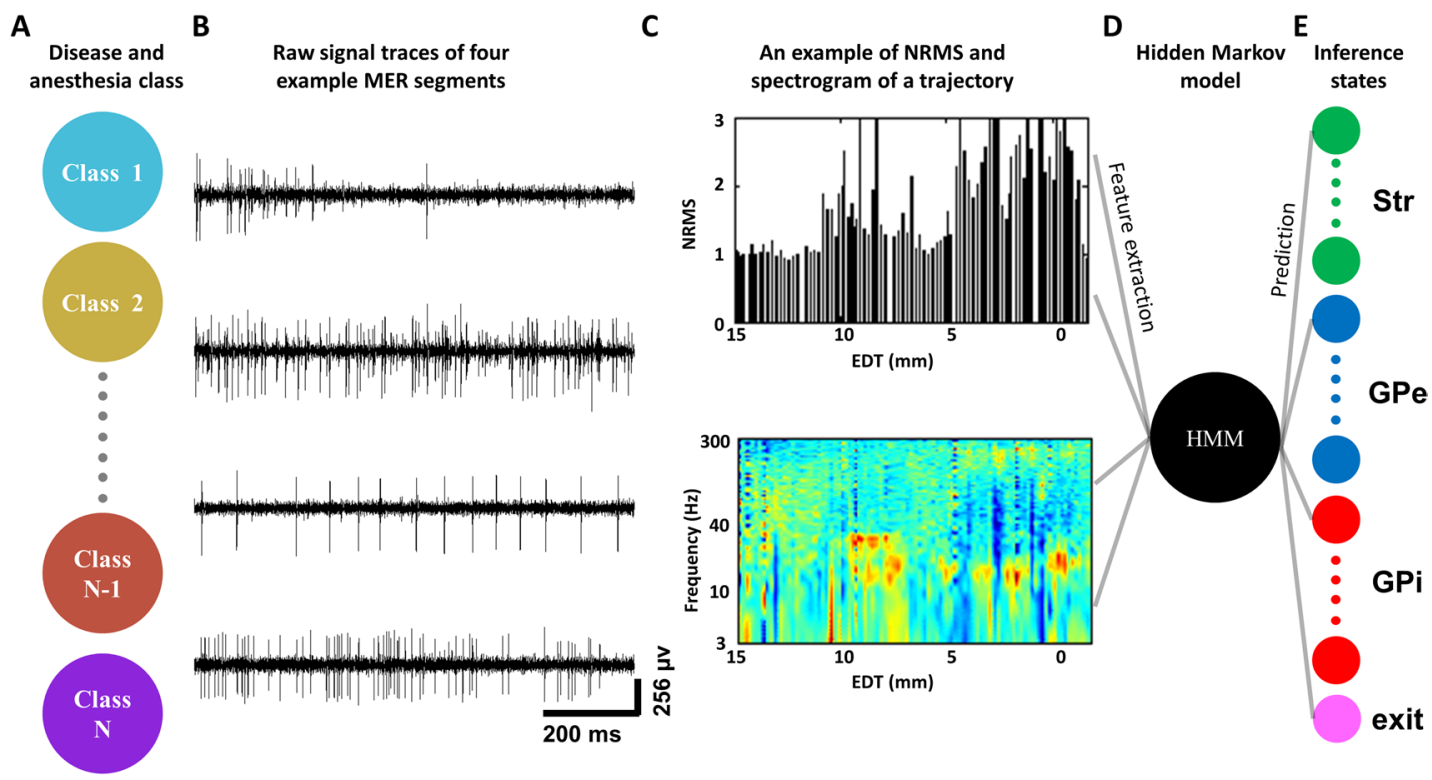

Figure 1. Visualization of the classification framework. Data flow is from left to right: (A) the disease and anesthesia class is selected intraoperatively. The classes consist of PD, genetic and non-genetic dystonia in anesthetized and awake patients. (B) The four raw signal traces (out of 106 recorded traces at various depths of one trajectory in the left hemisphere) along the trajectory (a single MER track) from a single PD patient illustrate the unit activity in the regions of striatum (injury potentials), GPe, border cell and GPi. (C) An example of the NRMS (top) and spectrogram (bottom) of the same trajectory as in (B), plotted in relation to the estimated distance from the target (EDT). (D) The features are extracted sequentially from the raw multi-unit activity recorded by the microelectrode at each electrode depth and fed into the HMM as an input observation. (E) The HMM computes the most likely inference state per MER, generating a sequence of states (for example, Str...Str GPe...GPe GPi...GPi exit). The inference states enable real-time decisions as to electrode placement. The power spectral density color-scale represents 10 log 10 (power spectral density power/average power spectral density power) per EDT. EDT = estimated distance to target (defined as the ventral border of the lateral-posterior portion of the GPi target according to preoperative imaging). NRMS = normalized root mean square. $\mathrm{HMM}=$ hidden Markov model.

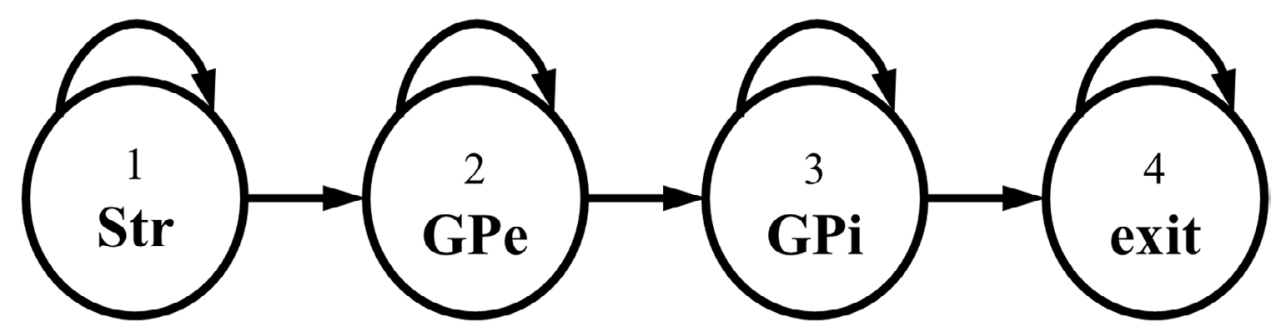

Figure 2. The left-right HMM procedure with four possible states. Arrows represent the three possible state transitions (striatum$\mathrm{GPe}, \mathrm{GPe}-\mathrm{GPi}$, and GPi-exit) and the possibility of staying in the same state with no transition (reflexive arrows).

non-genetic and genetic dystonia $N-1=32,46$ and 35 ), and then tested on the excluded trajectory (with no assumption of its sequence) by comparing the inferred HMM state transitions to the trajectory's known state transitions. The HMM inference state sequence was calculated as the most probable sequence beginning with the HMM in the striatum (state 1) before the first observation (using the Viterbi algorithm) [40]. This method was repeated $N(33,47$ and 36) times, thus testing each trajectory individually across all three diseases. The resulting mean (of 33, 47 and 36 trajectories) HMM transition and emission matrices are presented in the supplementary information table S3.

Second, we validated the machine learning algorithm against three electrophysiologists' manual detection of ten patients in the operating room. We incorporated the pallidal navigation algorithm into three electrophysiological systems (at three medical centers) for intraoperative use. The test dataset was composed of 27 trajectories (six Parkinson's disease patients, two genetic and two non-genetic dystonia patient). This dataset included some MER segments collected when active and passive limb movement were tested; however, the HMM was not updated with limb movement data during the real-time validation. Thus, the algorithm did not use the changes in MER related to active or passive movement as additional input (i.e. these movements were treated as were the other spontaneous movements during the recording), nor did it use the experts' interpretation of approaching the target. 


\section{Statistical analysis}

All statistics and analysis were performed in MATLAB (version 7.5 and R2013a) using both the machine learning and statistics toolbox and custom software. An unpaired, one-tailed Student's t-test was used to test the hypothesis that the mean microelectrode trajectory times were shorter in surgeries using our machine learning algorithm than in surgeries without the algorithm. The null hypothesis was that the machine learning algorithm does not reduce the mean microelectrode trajectory time. The alternative hypothesis was that the algorithm would reduce the microelectrode trajectory time. The accuracy of the algorithm was estimated via the reliability versus distance measure (between expert electrophysiologists' border estimations and the HMM output) by varying the criterion distance values of the detection at $0.1 \mathrm{~mm}$ increments. The statistics presented in this report, if not specified otherwise, are the mean \pm standard error of the mean (SEM).

\section{Results}

\section{Oscillatory characteristics of dystonia classes}

The mean power spectra and NRMS of the MER segments were calculated in the striatum, GPe, and GPi of the four dystonia classes (figure 3(A), awake and lightly anesthetized non-genetic and genetic dystonia, $n=2380,2202,1752$ and 2556 MER segments, respectively; a summary of the dataset is presented in supplementary table S2). The mean power spectra of the striatum in both the non-genetic and genetic dystonia classes under light anesthesia (figure 3(A), light green) manifested a unique signature: an alpha (8-12 Hz) oscillation, most likely due to light nitrous oxide and isoflurane or sevoflurane anesthesia [43]. In contrast, the GPe in both genetic and non-genetic dystonia (figure 3(A), light and dark blue) displayed relatively less alpha and beta-oscillatory activity. The power spectra of the GPi in the non-genetic awake dystonia class (figure 3(A) left, dark red) exhibited a peak in the high beta range $(20-30 \mathrm{~Hz})$. In contrast, the power spectra of the GPi in the genetic dystonia class in both the lightly anesthetized and awake patients displayed decreased beta-oscillatory activity.

\section{Awake versus anesthetized dystonia classes}

To evaluate the number of classes required for the classification system, we calculated the $\mathrm{L}_{1}$-distance (see Methods) of the mean relative power spectra (calculated on samples in the $3-70 \mathrm{~Hz}$ range) and NRMS. The distances were calculated between each region (for example, the striatum) to all other regions (for example, striatum, GPe and GPi) for the nongenetic and genetic dystonia classes. The three possible permutations of light general anesthesia (GA) and awake (Aw) groups can be seen in figures $3(\mathrm{~B})$ and $(\mathrm{C})$ (i.e. GA versus Aw, Aw versus Aw, GA versus GA). Across all four panels, the GA versus Aw group corresponded to the minimal distance values (shortest bars) in comparison to both Aw versus Aw and GA versus GA. This indicated there were minimal differences between the light GA versus Aw dystonia classes for both the PSD (in the 3-70 Hz range; figure 3(B)) and NRMS (figure 3(C)). Similar results were obtained for the power spectral density in the $3-300 \mathrm{~Hz}$ range. We therefore merged the Aw and light GA cases for the two (non-genetic and genetic) dystonia classes and reduced the number of dystonia disease classes to two different dystonia classes instead of four.

\section{Characteristics and spatial distribution of neural activity in the three classes of disease}

The NRMS and power spectra were calculated across the striatum, GPe and GPi in PD, non-genetic and genetic dystonia as a function of the distance from the ventral border (figure 4; $n=2884,4582$, and 4308 MER segments, respectively, averaged across electrode trajectories). Plotting the striatum spectrogram-by-depth demonstrated a similar spatial oscillatory distribution across all three classes of disease (figure 4, first column). The transition into the GPe was characterized by high tonic firing rates and increased background activity, which is indicated by the increased NRMS (figure 4, second column). The spatial distribution with low-frequency activity for both the GPe and GPi was nearly identical across genetic and non-genetic dystonia patients (figures 4 (B) and (C), second and third columns). In contrast, the GPe and GPi in PD exhibited tremor (4-5 Hz) frequency oscillation (figure 4(A), second row, second and third columns). The beta-oscillatory activity $(12-30 \mathrm{~Hz}$, with a maximal peak at $15 \mathrm{~Hz})$ in GPi PD was found primarily in the ventral portion of the trajectory (figure 4(A), second row, third column). Similar oscillatory activity can be seen in the mean plots (figure 4, right column, averaged across all patients and electrode trajectories). These results are consistent with local field potential studies $[15,17,44]$, which have described the ventro-lateral spatial location of beta and theta $(4-8 \mathrm{~Hz})$ oscillatory activity.

The mean relative PSDs of the spiking activity in GPi PD exhibited increased activity in the tremor (4-5 $\mathrm{Hz}$ ) frequency, beta-oscillatory activity and 150 to $250 \mathrm{~Hz}$ range, in comparison to the genetic and nongenetic dystonia (figure 5, third column, orange trace). The GPe PD exhibited low $(9-20 \mathrm{~Hz})$ and high beta (20-35 Hz) oscillatory activity (figure 5, second column, orange trace). In contrast to PD, the GPe and GPi in both genetic and non-genetic dystonia (figure 5, second and third columns) demonstrated relatively lower beta-oscillatory activity. This is in line with previous studies that have described different pallidal characteristics in PD and dystonia patients [15-17, 44-48]. The recordings in the striatum exhibited nearly identical activity across all three classes of disease (figure 5, first column). 

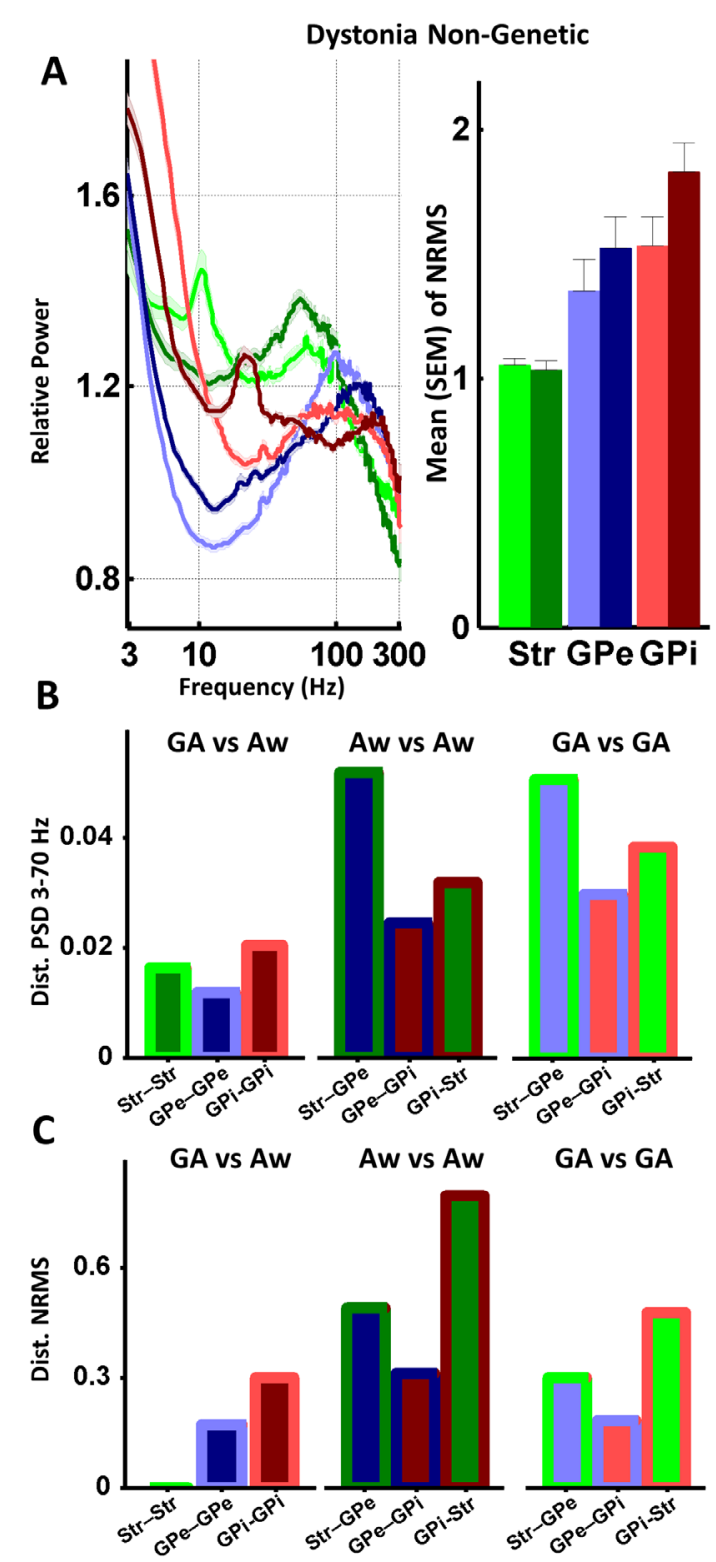

Figure 3. Minimal differences between awake and lightly anesthetized dystonia classes (A) the mean relative power spectral densities and the mean NRMS of non-genetic dystonia (left, first and second panels; represent awake (Aw) and light general anesthesia (GA), 2380 and 2202 MER segments, respectively) and genetic dystonia (right, third and fourth panels; represent Aw and GA, 1752 and 2556 MER segments, respectively). Color code: Str GA (light green), Str Aw (dark green), GPe GA (light blue), GPe Aw (dark blue), GPi GA (light red), GPi Aw (dark red). The mean power spectral density is plotted on a logarithmic scale on the $x$-axis. Color shading and error bars denote SEM. (B) and (C), each of the four panels show the distances between all the possible classes. The four panels represent the same type of class comparison: GA versus Aw, Aw versus Aw, GA versus GA. The distance is calculated between each region (for example, Str) to all other regions (for example, Str, GPe and GPi) corresponding to GA and Aw. (B) The distance is defined as the $l_{1}$-distance; i.e. the sum of the absolute differences in spectral density (calculated on samples in the 3-70 $\mathrm{Hz}$ range of top panel figure (A)). Similar results were obtained for the 3-300 Hz range. (C) Similar to (B), but for NRMS. Aw = awake; $\mathrm{GA}=$ light general anesthesia.

Power spectra features for pallidal border detection NRMS values calculated from the MER segments were effective in detecting the striatum-GPe and GPiexit borders because of the increase and decrease in the NRMS (figures 4, 6 and supplementary material figure S1). In contrast, detection of the GPe-GPi borders was more difficult, since often there was no sharp increase or decrease in NRMS between these structures. However, visual inspection of the mean power spectra (figure 4, right column) did reveal differences between spectra in the $3-300 \mathrm{~Hz}$ domain. To determine quantitatively which part of the spectral domain enables the best discrimination between regions, we calculated the absolute difference in the mean power spectra. We then examined eight different frequency bands (approximately logarithmically equally distributed) bands along the frequency axis (the discrimination matrix is presented in supplementary table S4). Frequency bands at 3-30 $\mathrm{Hz}, 8-35 \mathrm{~Hz}, 30-95 \mathrm{~Hz}$ and $180-245 \mathrm{~Hz}$ provided the greatest discrimination for the striatum-GPe and GPeGPi transitions (supplementary material figure S2). These frequency bands were used to calculate the input features of the HMM. 

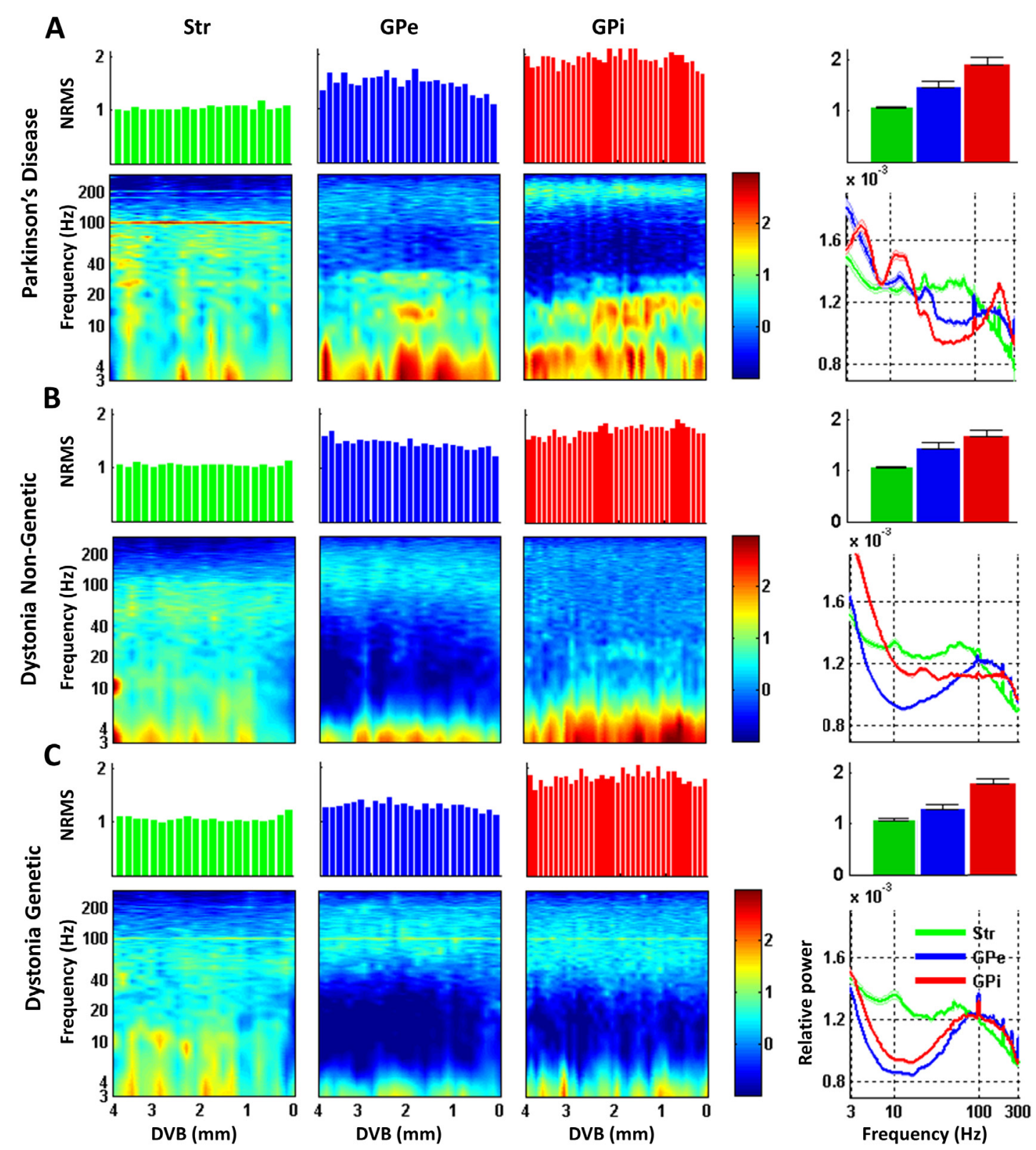

Figure 4. Differences and similarities in spatial distribution of neural activity between three classes of disease. The mean NRMS and power spectral density (three left columns) of the striatum (Str, green), GPe (blue) and GPi (red) plotted as a function of the distance from the ventral border (DVB; averaged across all patients) of each nucleus in (A) Parkinson's disease, (B) non-genetic and (C) genetic dystonia (calculated from $n=2884,4582$, and 4308 MER segments, respectively). The power spectral density color-scale represents $10 \log 10$ (power spectral density power/average power spectral density power) per DVB. The rightmost column represents the mean of the NRMS and relative power spectral density that was averaged across all patients and electrode trajectories (error bars denoting SEM). The relative power is plotted against the frequency for the three regions in the three classes of disease. The mean power spectral density is plotted with a logarithmic scale of frequency on the X-axis. Color shading denotes SEM. $\mathrm{DVB}=$ distance from the ventral border of each nucleus.

\section{Classification of pallidal borders for each of the} three classes of disease

The mostly flat NRMS (figure 4) allowed us to assume a chain of stationary MER segments within each structure. Under this assumption, we utilized an HMM to model the space-varying stochastic neural activity as a probabilistic Markovian chain of four stationary states corresponding to each structure. The HMM uses MER features and trajectory history to enable real-time decisions about electrode placement. The use of trajectory history in addition to the MER features enable the HMM to reduce variability in the inference of the location based on firing characteristics of the neurons encountered along the striato-pallidal trajectory. Based on our finding of minimal differences between the awake and light anesthesia dystonia classes (figure 3), we restricted the training and validation of HMM to three classes of disease: PD, non-genetic dystonia and genetic dystonia. Figure 6 presents a typical classification example of pallidal borders for each of the three classes of disease.

The reliability versus distance was tested using both leave-one-trajectory-out cross-validation and against three electrophysiologists in the operating 

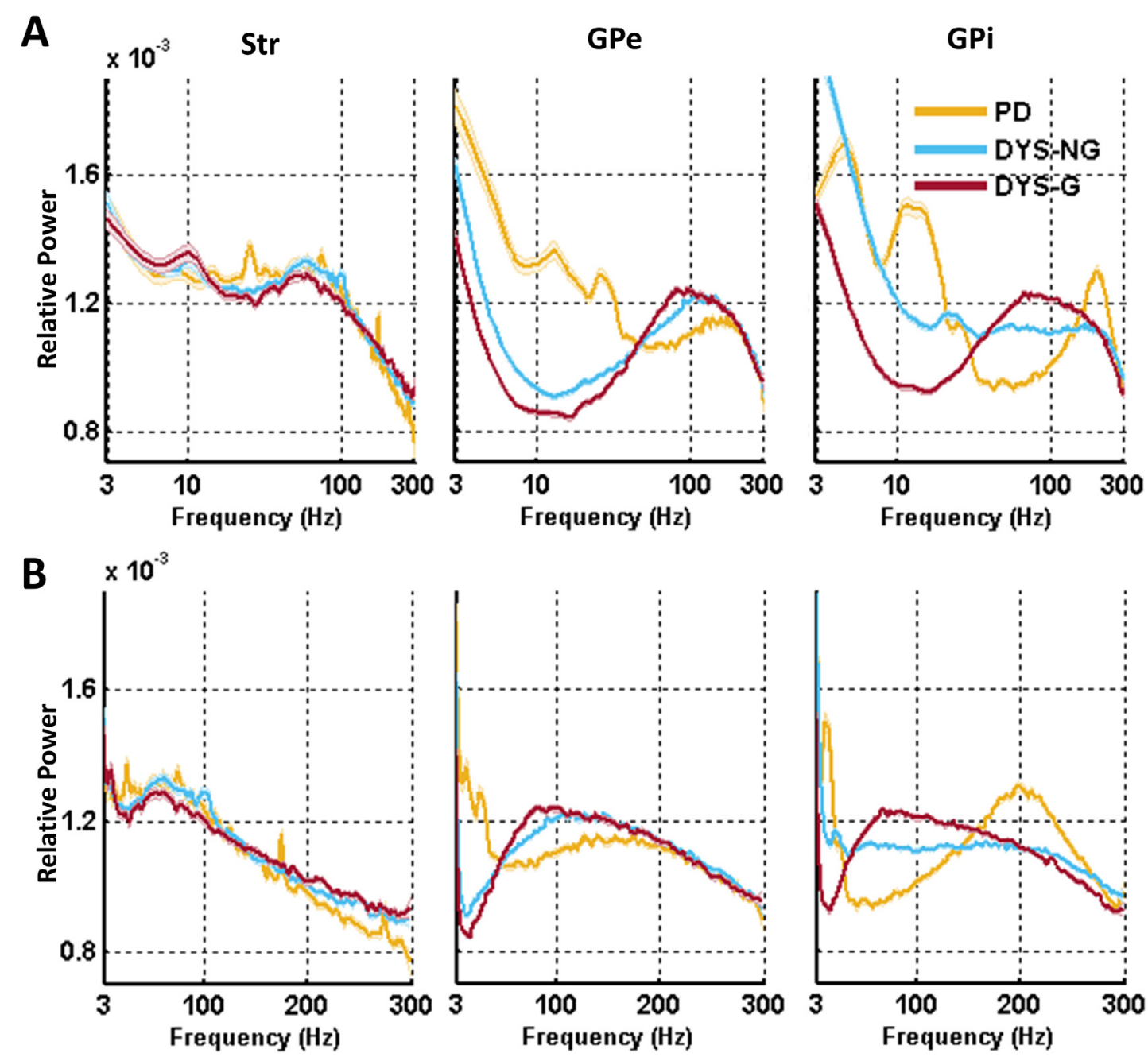

Figure 5. A distinct $\beta$ band in the GPe and GPi of Parkinson's disease patients. (A) The power spectral density plot for the striatum (Str, left column), GPe (middle column) and GPi (right column) was averaged across all patients and electrode trajectories (shaded regions denoting SEM). The power spectral density plotted as a function of the frequency, with a logarithmic scale plotted across all three classes of disease: Parkinson's disease (orange), dystonia non-genetic (cyan), dystonia genetic (maroon) calculated from $n=2884,4582$, and 4308 MER segments, respectively. (B) The same as in A, but with a linear scale plot of the $x$-axis. $\mathrm{PD}=$ Parkinson's disease; $\mathrm{DYS}-\mathrm{NG}=$ dystonia non-genetic DYS-G = dystonia genetic.

room using newly collected data. The PD algorithm exhibited a robust demarcation of the Str-GPe and GPi-exit borders which achieved $84 \%$ and $95 \%$ accuracy within a $0.3 \mathrm{~mm}$ distance, respectively, using leave-one-trajectory-out cross-validation (figure 7(A), left and right columns). The test using new data shows $95 \%$ and $83 \%$ accuracy within $0.3 \mathrm{~mm}$ distance, respectively (figure $7(\mathrm{~B})$, left and right column). In contrast, many trajectories lacked a clearly defined GPe-GPi border (figures 7(A) and (B), middle column). The PD algorithm achieved $78 \%$ and $45 \%$ accuracy within a $0.3 \mathrm{~mm}$ distance on the two validation measures, respectively. These results are not surprising given that the expert electrophysiologist was uncertain as to the exact location of the GPe-GPi border in $47 \%$ (54/116) of the training trajectories, which may be due to the similarity between the GPe and GPi power spectra (figure 3, right column), white matter gaps of $>1 \mathrm{~mm}$ between the GPe and GPi, uninterrupted GPe-GPi transitions and cases with no clear short transient reduction and increase in the NRMS (figure 6, top row, middle and right column) [21]. For example, in PD and non-genetic dystonia (figure 6, left and middle column), the HMM transition inference concurred (error $<0.3 \mathrm{~mm}$ ) with the expert's decision for the Str-GPe and GPi-exit transitions (solid red and black lines), but overshot the GPe-GPi transition (error $>0.3 \mathrm{~mm}$ dot-dash red and black lines).

The lower performance of the HMM for the dystonia patients (figure $7(\mathrm{~A})$ ) was most likely due to the small number of trajectories and the variability in the physiology across the dystonia patients. The HMM provided the same detection reliability results when limb movement-related MER segments (i.e. data when active and passive limb movements were tested) were excluded from the validation dataset. The detection reliability of the striato-pallidal borders is novel and therefore does not have a reference for comparison, but showed results similar to the STN entry and exit detections [49]. 


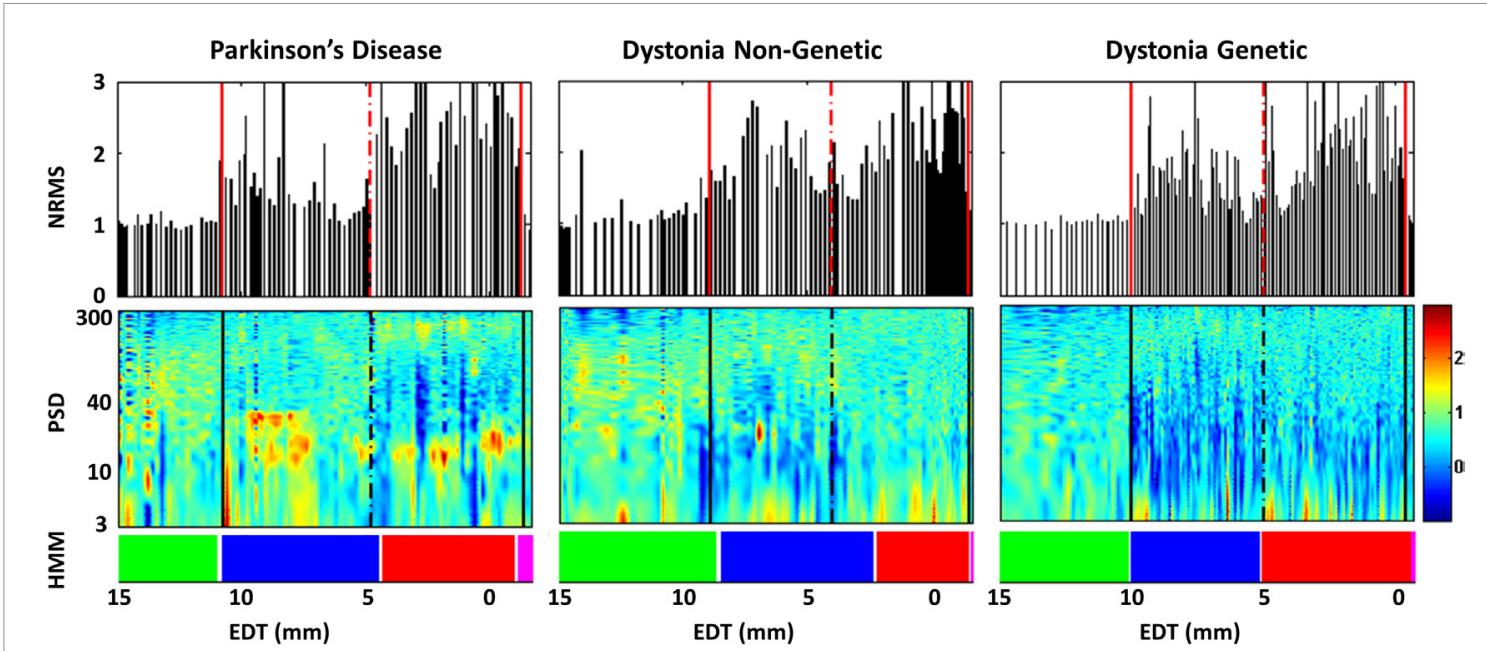

Figure 6. A classification example of pallidal borders for each of the three classes of disease. Top row represents the trajectory's NRMS. Second row represents the PSD of the same trajectory. The red (top row) and black (second row) solid vertical lines indicate Str-GPe and GPi-exit transitions, whereas the dot-dash lines indicate the GPe-GPi boundary defined by an expert electrophysiologist. Bottom row represents the HMM inference states as a function of the EDT; the green, blue, red and pink rectangles represent the striatum, GPe, GPi, and exit regions, respectively. The PSD color-scale represents 10 log10 (power spectral density power/average power spectral density power) per EDT. The three sample trajectories illustrate the classification of striatopallidal borders in the three diseases: Parkinson's disease (left column; 106 MER segments), non-genetic dystonia (middle column; 113 MER segments) and genetic dystonia (right column; 132 MER segments). The total recording time of a single DBS track of the example trajectories from left to right columns: $524 \mathrm{~s}, 678 \mathrm{~s}$ and $792 \mathrm{~s}$. The HMM running time from left to right column: $130 \mathrm{~ms}$, $164 \mathrm{~ms}$ and $187 \mathrm{~ms}$. MER = micro-electrode recording.

The machine learning algorithm reduced the time it took the expert electrophysiologist to determine the striato-pallidal borders, since the duration with the algorithm was shorter than without it. The mean microelectrode trajectory time per single $15 \mathrm{~mm}$ trajectory with and without the algorithm was $26 \pm 1.3$ and $36 \pm 1.5 \mathrm{~min}$ (mean $\pm \mathrm{SEM}$ ), for $n=27$ and 116 trajectories, respectively (unpaired, one-tailed t-test $P<0.05)$. Note that it is likely that the border demarcation was more accurate with the HMM; however, here we could not evaluate the difference in accuracy. It took 130 milliseconds in real-time for the machine learning algorithm to compute a single DBS track and determine the striato-GPe-GPi borders (Intel Core I5 $2410 \mathrm{M}$ processor with $4 \mathrm{~GB}$ RAM). The time to analyze each subsequent MER was one millisecond per MER, making the HMM algorithm a practical method for use during DBS surgery.

\section{Discussion}

The goal of this study was to generate a robust procedure for GPi localization that can inform the surgical team intraoperatively, in real-time, of the electrode position. Here, we derived a machine learning approach to analyze MER segments during surgery for implanting a DBS lead in the GPi. To determine whether the machine learning algorithm could accurately detect the GPi, we utilized a large anatomically annotated database of striato-pallidal MER segments from operated awake PD patients, as well as awake and lightly anesthetized genetic and nongenetic dystonia patients. We tested its performance against three electrophysiologists in the operating rooms of three hospitals on data that were not used to develop the machine learning algorithm.

\section{Awake versus anesthesia dystonia classes}

Several recent studies have reported differences in pallidal firing rates between dystonia classes under volatile anesthetics $[50,51]$. The results of this study extend previous work by comparing the spectral oscillatory changes in neural activity within the striatum, GPe and GPi between awake and lightly anesthetized genetic and non-genetic dystonia classes (figure 3(A). Our MER-derived results are consistent with previous findings of oscillatory patterns of pallidal local field potentials in dystonia patients [15, $16,44,45,52]$. Using the $\mathrm{L}_{1}$-distance measure, we determined that the awake and the light anesthesia (0.3-0.6 MAC range) dystonia classes could be merged (figures 3(B) and (C)). Thus, although anesthesia may reduce pallidal neuronal oscillations across all dystonia classes [25,26], detectable relative differences in neural oscillations between genetic dystonia and non-genetic classes were preserved.

\section{Striatal alpha oscillation under light anesthesia}

A growing body of evidence suggests that anesthetics induce oscillations that alter or disrupt the normal brain's information processing [53, 54]. Because the cortex is the major afferent to the striatum [55], profound changes in neural activity in the cortex, which are reflected in major changes in the scalp electroencephalogram (EEG), might be transmitted to the striatum. Our MER-derived results that reveal alpha oscillations in the striatum under light anesthesia (figure $3(\mathrm{~A})$ ) are consistent with findings 
showing an increase in oscillatory EEG power in the alpha frequency range under isoflurane or sevoflurane anesthesia [43]. Even though striatal neurons send inhibitory projections to the GPe and GPi [56], we found that striatal neurons did not propagate these alpha oscillations to these structures (figure 3(A)). This may be explained by studies in the non-human primates indicating that the output activity of the pallidum (or basal-ganglia downstream structures) does not reflect striatal neural activity [57]. Therefore, abnormal oscillatory activity within striatum, under light anesthesia, did not propagate to the GPe and GPi. Another possibility is that the oscillatory activity in the different domains of the striatum (e.g. ventral and dorsal striatum) is not the same. We may therefore have recorded the neural activity from those parts of the GPe and GPi that receive information from domains of the striatum that are not involved in alpha oscillations.

\section{Differences in striato-pallidal oscillatory activity between the three classes of disease}

Several studies have reported lower mean discharge rates in GPi neurons of dystonia patients compared to the non-human primates or humans with PD $[45,47]$. For the purposes of this study, we chose to focus on power spectra and the RMS of the MER multi-unit (background) activity rather than the discharge rate, since well isolated single-unit studies are too timeconsuming and computationally demanding to be performed in a real-time intraoperative scenario. We compared the spatial distribution of the oscillatory neural activity within the striatum, GPe and GPi between the three classes of disease (figure 4). We found differences in striato-GPe-GPi oscillatory activity between the $\mathrm{PD}$, genetic dystonia and nongenetic dystonia classes (figure 5). These differences could be used in the future as electrophysiological target-specific biomarkers to optimize therapy in adaptive DBS control systems [16, 58].

\section{Difficulty in determining the GPe-GPi transitions}

The method described in this article varies in its capability to predict GPe- and GPi- entry and exit. Whereas the striatum-GPe and GPi-exit were usually clear and characterized by a distinguishable increase and fall of the NRMS, respectively, the GPe-GPi transitions were harder to detect in some trajectories (figure 7). The GPe is separated from the GPi by a thin layer of myelinated fibers called the medial medullar lamina [59]. The GPe-GPi transition can therefore be detected by a drop in previous neuronal activity and the recording of border cells in its vicinity. However, the transition from the GPe to GPi was not always clear to the expert or to our machine learning algorithm (figure 7 middle column). This may have been due in part to the sparsely distributed neurons along the pallidum and the variability in the firing characteristics between the GPe and GPi in the MER dataset [21]. Clinically, the GPe-GPi boundary reliability is probably not very important. This is because, for accurate DBS lead placement, the critical boundary is the GPi-exit border where the PD algorithm achieved $83 \%$ accuracy (on the test data) within a $0.3 \mathrm{~mm}$ distance (an error considered 'acceptable' by our team; figure 7(B) right column). Usually, trajectories that traverse $>10 \mathrm{~mm}$ in the pallidum or $>3.5 \mathrm{~mm}$ of GPi enable the implanting of the DBS lead in the optimal location (with two contacts inside the GPi), even without identifying the low border of GPi.

\section{Choosing the 'right' machine learning algorithm}

The need for a real-time simple method (to understand, maintain and deploy in different currently available intraoperative MER software) was a crucial guideline when choosing the machine learning algorithm to work with. Because we do not advance the microelectrode beyond the ventral border of the GPi, the number of MER segments recorded in the white matter (exit) was about 10-fold smaller than that of other states (Str, GPe and GPi). For example, multiclass support vector machine (SVM) techniques typically treat all states equally; hence, it is not well suited to our imbalanced datasets, and may cause a bias in the decision boundary of the SVM classifier [60].

Previous studies have used deep neural network and hybrid SVM-HMM systems for offline state detection with large datasets (i.e. 150-750 independent trials) $[61,62]$. In contrast, we used an HMM to enable accurate GPi confirmation that does not require the presence of an expert physiologist intraoperatively, in real-time. Our machine learning algorithm has four main advantages: it does not rely on spike detection and sorting procedures, state prediction is estimated with an imbalanced limited dataset (e.g. 33 independent trajectories for PD), only a short signal recording is required, and finally it is unaffected by variability in electrode properties and the noisy environment of the operating room. However, our method is constrained by the small dataset. The results may be compared to deep neural network and hybrid systems if sufficient training examples exist. There are surgical procedures, such as STN-DBS, for which the deep neural network approaches (e.g. long short-term memory networks; LSTM $[63,64])$ may be effective. This is because STNDBS datasets tend to be more balanced and consists of a much larger dataset (in most medical centers) $[41,65]$. In the future, the LSTM could be used for training on the NRMS, EDT and power spectra data [62] to classify striato-pallidal regions in real-time.

\section{Some limitations of the machine learning algorithm} Our study also has several limitations that should be addressed before the algorithm is adopted for widespread use. First, the machine learning algorithm was tested on a small number of patients intraoperatively in real-time (i.e. 27 trajectories in 10 patients), due to the relatively low rate of GPi-DBS procedures in Israel (most procedures target the STN). 

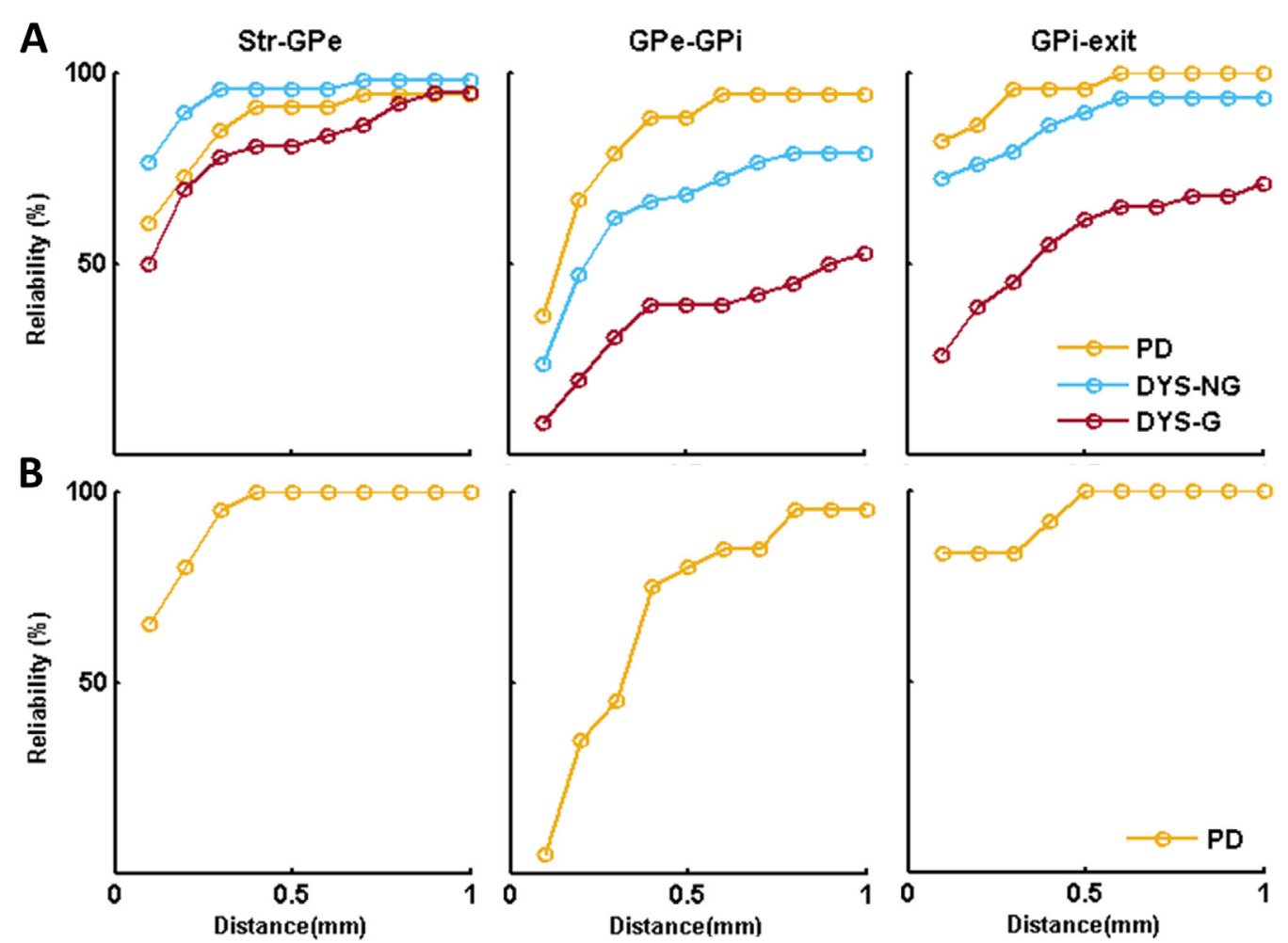

Figure 7. Striato-pallidal border demarcation performance (A) The HMM exhibited reliable striato-pallidal border demarcation when tested with leave-one-trajectory-out cross-validation. Classification results were obtained by varying the threshold distance values of the detection at $0.1 \mathrm{~mm}$ increments. For example, for the Str-GPe border at a distance of $0.3 \mathrm{~mm}$ the classification results achieved $84 \%$ accuracy for the PD algorithm. The HMM was effective in detecting the Str-GPe and GPi-exit borders for all three classes of diseases: Parkinson's disease (orange), dystonia non-genetic (cyan) and dystonia genetic (maroon). In contrast, the determination of the GPe-GPi transition boundary was more ambiguous and difficult to evaluate for both the electrophysiologists and the HMM. The classification was restricted to each class: Parkinson's disease ( $n=33,33$ and 23 for Str-GPe, GPe-GPiGPe-GPi and GPi-exit borders, respectively), non-genetic ( $n=47,47$ and 30 for Str-GPe, GPe-GPi and GPi-exit borders, respectively) and genetic dystonia ( $n=36,36$ and 31 for Str-GPe, GPe-GPi and GPi-exit borders, respectively). (B) The HMM produced similar results when tested against three expert electrophysiologists on three different recording systems (i.e. distinct operating rooms). The real-time test dataset in the operating room included: Parkinson's disease ( $n=20,20$ and 12 for Str-GPe, GPe-GPi and GPi-exit borders, respectively), non-genetic ( $n=3,3$ and 1 for Str-GPe, GPe-GPi and GPi-exit borders, respectively) and genetic dystonia ( $n=4,4$ and 2 for Str-GPe, GPe-GPi and GPi-exit borders, respectively). The lower number of GPi-exit transitions was due to the practice we and other medical centers implement, which is: not to advance the microelectrode beyond the $0 \mathrm{~mm}$ estimated distance to the target or to the ventral border of the GPi. We only show the test performance results for the Parkinson's disease patients because of the small intraoperative dataset for dystonia. Similar quantitative results were obtained for three trajectories of non-genetic and four trajectories of genetic dystonia patients. The leave-one-trajectory-out cross-validation curves are smoother because of the larger dataset. $n=$ the number of transitions between structures. PD $=$ Parkinson's disease; DYS-NG $=$ dystonia non-genetic; DYS-G = dystonia genetic.

Second, the entry (i.e. the white-matter-striatum transition) was not tested because our intraoperative recordings started at the level of the striatum rather than white-matter in all three medical centers. This decision reflects the desire to minimize operating time, especially in awake patients and our view that identification of the striatum-pallidum transition is more critical for optimal GPi navigation. Third, we excluded 'poor' trajectories from the training and validation dataset which for a variety of reasons (e.g. deep anesthesia) did not yield any neural activity. Although all our recordings started $15 \mathrm{~mm}$ above the calculated target and traversed $>7 \mathrm{~mm}$ of pallidum, our dataset included a variety of trajectories, such as a long GPe with a short GPi, no drop or a very long gap in neural activity between the GPe and GPi. This affected the GPe-GPi and GPi-exit border demarcation performance (figure 7).
Another potential limitation is that the current approach may not allow GP border prediction if the recordings start at the level of the pallidum. To enable HMM training on the trajectory history and comparisons across trajectories, we normalized the RMS values by the relatively MER-silent striatum activity (figure 4) in the same trajectory (see Methods). This limits our HMM's ability to 'learn new trajectories' if not all medical centers start in the striatum. However, most centers that use MER usually start their GPi exploration at $15 \mathrm{~mm}$ above target, as described here. It is possible that other differences between medical centers may impose difficulties on the algorithm; thus, future research is needed to determine how models trained in one center can best be implemented in other centers with different protocols. This would be especially useful for centers with limited databases and a lack of experience in pallidal electrophysiology. 
What if the trajectory calculations were too lateral?

An issue that was not addressed in the present study is how to determine medial-lateral location. The GPi location differs across patients and is not easily visible pre-operatively in all cases on $\mathrm{MR}$ imaging. If the trajectory is too lateral, the trajectory might miss the entire GPi and only hit the GPe; if so, the method described here may not work, but the MER would still be very useful to the surgeon in deciding where to select another trajectory. The optimal trajectory in mediallateral terms will usually have approximately equal lengths of GPe and GPi. None of the trajectories in our study missed the GPi. Nonetheless, further research and data are needed to design an algorithm to overcome this eventuality. Macrostimulation, especially in the awake patient, can also provide information concerning distance from the internal capsule.

\section{The advantages of using MERs with a machine learning algorithm}

Some experts have argued against the utility of MER. This controversy remains unresolved. In our experience, as is the case for many other experts, MER is both safe and provides high resolution target verification [28]. One rationale for the case against MER is a lack of physiological expertise. In medical centers where there is no electrophysiologist in the operating room, the machine learning algorithm can provide assistance to the neurosurgeon. Machine learning algorithms are becoming increasingly commonplace in the operating room as well as in other fields. Automating navigation and detection of brain structures intraoperatively is an important area for future investigation, and includes identifying optimal implant target locations. Machine learning can also help the neurosurgeon decide which MER track to implant and determine whether stimulating different portions of the target (i.e. ventral, dorsal, medial or lateral) will have different therapeutic benefits or side effects. We nevertheless feel that our machine learning algorithm can make a contribution to MER users, and encourage others to use it and improve their DBS outcomes.

\section{Conclusion}

Here we demonstrated the effectiveness and reliability of real-time, automatic intraoperative verification of the striatum-GPe, GPe-GPi and GPi-exit transitions during DBS surgery. An HMM trained on the trajectory history and characteristic features of striatal and pallidal activity performed on a par with all three experts in detecting striato-pallidal borders across the three classes of disease: PD, genetic dystonia and non-genetic dystonia. Further research is necessary to evaluate performance in different clinical settings, in order to validate this technique across the spatial distributions of neural activity encountered along the trajectory in the typical operating room setting. The HMM is agnostic to the type of raw data used and can be implemented easily in existing MER devices. The ability to classify striato-pallidal regions in real-time using our classification algorithm may shorten DBS surgery considerably. The machine learning algorithm approach considers all available example trajectories equally and finds transitions between nuclei that a human researcher may miss for example, due to bias towards a given interpretation. This method allows accurate GPi confirmation without the necessity of an expert physiologist.

\section{Acknowledgments}

We thank Zelma Kiss for fruitful discussions, Ido Strauss for allowing us to test the machine learning algorithm in the operating room at the Sourasky Medical Center, Ariel Tankus and Omer Zarchi for their help in electrophysiological recordings and striato-pallidal border determination, Salam Oukal, Ayman Yousef and Omer Naor for their assistance in incorporating the GPi navigation system into the Neuro Omega system for the intraoperative use in three medical centers, Odeya Marmor-Levin for her help in collecting patient data and the patients for agreement to participate in the study and authorizing the use of their electrophysiological recordings.

\section{Conflict of interest/financial disclosures}

Dr Hagai Bergman reports receiving a travel honorarium from Alpha Omega, Medtronic and Boston Scientific and serving as a consultant for Alpha Omega. Dr Zvi Israel reports serving as a consultant for Alpha Omega.

\section{Funding agencies}

This study was supported by the Magnet program of the Israel Innovation Authority of the Israel Ministry of Economy.

\section{Appendices}

Additional supplementary material is available in the online version of this article on the publisher's website.

\section{ORCID iDs}

Dan Valsky (1) https://orcid.org/0000-0002-2385-424X Hagai Bergman (10) https://orcid.org/0000-0002-24026673

\section{References}

[1] Anderson V C, Burchiel K J, Hogarth P, Favre J and Hammerstad J P 2005 Pallidal versus subthalamic nucleus deep brain stimulation in Parkinson disease Arch. Neurol. 62 554-60

[2] Okun M S et al 2009 Cognition and mood in Parkinson's disease in subthalamic nucleus versus globus pallidus interna 
deep brain stimulation: the COMPARE Trial. Ann. Neurol. 65 586-95

[3] Weaver F M et al 2012 Randomized trial of deep brain stimulation for Parkinson disease: thirty-six-month outcomes Neurology 79 55-65

[4] Odekerken V J et al 2016 GPi versus STN deep brain stimulation for Parkinson disease: three-year follow-up Neurology 86 755-61

[5] Ostrem J L and Starr P A 2008 Treatment of dystonia with deep brain stimulation Neurotherapeutics 5320-30

[6] Coubes P et al 2004 Electrical stimulation of the globus pallidus internus in patients with primary generalized dystonia: long-term results J. Neurosurg. 101 189-94

[7] Krause M, Fogel W, Kloss M, Rasche D, Volkmann J and Tronnier V 2004 Pallidal stimulation for dystonia Neurosurgery 55 1361-8

[8] Vidailhet M et al 2005 Bilateral Deep-brain stimulation of the globus pallidus in primary generalized dystonia New Engl. J. Med. 352 459-67

[9] Volkmann J et al 2012 Pallidal Deep brain stimulation in patients with primary generalised or segmental dystonia 5-year follow-up of a randomised trial Lancet Neurol. 11 1029-38

[10] SaengerV M et al 2017 Uncovering the underlying mechanisms and whole-brain dynamics of deep brain stimulation for Parkinson's disease Sci. Rep. 79882

[11] Rolston J D, Englot D J, Starr P A and Larson P S 2016 An unexpectedly high rate of revisions and removals in deep brain stimulation surgery: analysis of multiple databases Parkinsonism Relat. Disord. 33 72-7

[12] Okun M S et al 2005 Management of referred deep brain stimulation failures Arch. Neurol. 621250

[13] Wong S, Baltuch G H, Jaggi J L and Danish S F 2009 Functional localization and visualization of the subthalamic nucleus from microelectrode recordings acquired during DBS surgery with unsupervised machine learning J. Neural Eng. 6026006

[14] Zaidel A, Spivak A, Shpigelman L, Bergman H and Israel Z 2009 Delimiting subterritories of the human subthalamic nucleus by means of microelectrode recordings and a hidden Markov model Mov. Disord. 24 1785-93

[15] Neumann W-J et al 2017 A localized pallidal physiomarker in cervical dystonia Ann. Neurol. 82 912-24

[16] Wang D D et al 2018 Pallidal deep-brain stimulation disrupts pallidal beta oscillations and coherence with primary motor cortex in Parkinson's disease J. Neurosci. 38 4556-68

[17] Piña-Fuentes D et al 2019 The characteristics of pallidal lowfrequency and beta bursts could help implementing adaptive brain stimulation in the parkinsonian and dystonic internal globus pallidus Neurobiol. Dis. 121 47-57

[18] Reich M M et al 2019 Probabilistic mapping of the antidystonic effect of pallidal neurostimulation: a multicentre imaging study Brain 142 1386-98

[19] Pauls KA M et al 2017 Causes of failure of pallidal deep brain stimulation in cases with pre-operative diagnosis of isolated dystonia Parkinsonism Relat. Disord. 43 38-48

[20] Bjerknes S et al 2018 Multiple microelectrode recordings in STN-DBS surgery for Parkinson's disease: a randomized study Mov. Disord. Clin. Pract. 5 296-305

[21] Schiff S J, Dunagan B K and Worth R M 2002 Failure of singleunit neuronal activity to differentiate globus pallidus internus and externus in Parkinson disease J. Neurosurg. 97 119-28

[22] Lozano A M and Hutchison W D 2002 Microelectrode recordings in the pallidum Mov. Disord. 17 S150-4

[23] Vitek J L et al 1998 Microelectrode-guided pallidotomy: technical approach and its application in medically intractable Parkinson's disease J. Neurosurg. 88 1027-43

[24] DeLong M R 1971 Activity of pallidal neurons during movement J. Neurophysiol. 34 414-27

[25] Venkatraghavan L, Rakhman E, Krishna V, Sammartino F, Manninen P and Hutchison W 2016 The effect of general anesthesia on the microelectrode recordings from pallidal neurons in patients with dystonia J. Neurosurg. Anesthesiol. $28256-61$
[26] Hutchison W D, Lang A E, Dostrovsky J O and Lozano A M 2003 Pallidal neuronal activity: implications for models of dystonia Ann. Neurol. 53 480-8

[27] Thompson J A, Yin D, Ojemann S G and Abosch A 2017 Use of the putamen as a surrogate anatomical marker for the internal segment of the globus pallidus in deep brain stimulation surgery Stereotact. Funct. Neurosurg. 95 229-35

[28] Lozano C S et al 2018 Imaging alone versus microelectrode recording-guided targeting of the STN in patients with Parkinson's disease J. Neurosurg. 130 1789-2097

[29] Choi K S et al 2018 Impact of brain shift on subcallosal cingulate deep brain stimulation Brain Stimul. 11 445-53

[30] Khan M F, Mewes K, Gross R E and Škrinjar O 2008 Assessment of brain shift related to deep brain stimulation surgery Stereotact. Funct. Neurosurg. 86 44-53

[31] Halpern C H, Danish S F, Baltuch G H and Jaggi J L 2008 Brain shift during deep brain stimulation surgery for Parkinson's disease Stereotact. Funct. Neurosurg. 86 37-43

[32] Umemura A et al 2003 Deep brain stimulation for movement disorders: morbidity and mortality in 109 patients J. Neurosurg. 98 779-84

[33] Castrioto A et al 2016 Anesthesia reduces discharge rates in the human pallidum without changing the discharge rate ratio between pallidal segments Eur. J. Neurosci. 44 2909-13

[34] Zaidel A, Spivak A, Grieb B, Bergman H and Israel Z 2010 Subthalamic span of $\beta$ oscillations predicts deep brain stimulation efficacy for patients with Parkinson's disease Brain 133 2007-21

[35] Zaidel A, Arkadir D, Israel Z and Bergman H 2009 Akinetorigid versus tremor syndromes in Parkinsonism Curr. Opin. Neurol. 22 387-93

[36] Moran A, Bar-Gad I, Bergman H and Israel Z 2006 Real-time refinement of subthalamic nucleus targeting using Bayesian decision-making on the root mean square measure Mov. Disord. 21 1425-31

[37] Moran A, Bergman H, Israel Z and Bar-Gad I 2008 Subthalamic nucleus functional organization revealed by parkinsonian neuronal oscillations and synchrony Brain 131 3395-409

[38] Moran A and Bar-Gad I 2010 Revealing neuronal functional organization through the relation between multi-scale oscillatory extracellular signals J. Neurosci. Methods 186 116-29

[39] Eddy S R 2004 What is a hidden Markov model? Nat. Biotechnol. 22 1315-6

[40] Rabiner L R 1989 A tutorial on hidden Markov models and selected applications in speech recognition Proc. IEEE 77 257-86

[41] Valsky D et al 2017 Stop! border ahead: automatic detection of subthalamic exit during deep brain stimulation surgery Mov. Disord. 32 70-9

[42] Foltynie T, Jahanshahi M and Hariz M 2018 Association of optic pathways and brain structure with deep brain stimulation of the nucleus basalis of meynert for parkinson disease dementia-reply JAMA Neurol. 75896

[43] Purdon P L, Sampson A, Pavone K J and Brown E N 2015 Clinical electroencephalography for anesthesiologists Anesthesiology 123 937-60

[44] Silberstein P et al 2003 Patterning of globus pallidus local field potentials differs between Parkinson's disease and dystonia Brain 126 2597-608

[45] Weinberger M et al 2012 Oscillatory activity in the globus pallidus internus: comparison between Parkinson's disease and dystonia Clin. Neurophysiol. 123 358-68

[46] Tang J K H et al 2007 Neuronal firing rates and patterns in the globus pallidus internus of patients with cervical dystonia differ from those with Parkinson's disease J. Neurophysiol. 98720-9

[47] Starr P A et al 2005 Spontaneous pallidal neuronal activity in human dystonia: comparison with Parkinson's disease and normal macaque J. Neurophysiol. $933165-76$

[48] Starr P A et al 2006 Microelectrode-guided implantation of deep brain stimulators into the globus pallidus internus for 
dystonia: techniques, electrode locations, and outcomes J. Neurosurg. 104 488-501

[49] Thompson JA et al 2018 Semi-automated application for estimating subthalamic nucleus boundaries and optimal target selection for deep brain stimulation implantation surgery J. Neurosurg. 1-10

[50] Welter M-L et al 2015 Pallidal activity in myoclonus dystonia correlates with motor signs Mov. Disord. 30 992-6

[51] McClelland V M et al 2016 Differences in globus pallidus neuronal firing rates and patterns relate to different disease biology in children with dystonia J. Neurol. Neurosurg. Psychiatry 87 958-67

[52] Moll C K E et al 2014 Asymmetric pallidal neuronal activity in patients with cervical dystonia Frontiers Syst. Neurosci. 815

[53] Purdon P L et al 2013 Electroencephalogram signatures of loss and recovery of consciousness from propofol Proc. Natl Acad. Sci. 110 E1142-51

[54] Cimenser A et al 2011 Tracking brain states under general anesthesia by using global coherence analysis Proc. Natl Acad. Sci. $1088832-7$

[55] Kemp J M and Powell T P 1970 The cortico-striate projection in the monkey Brain 93 525-46

[56] Haber S N 2003 The primate basal ganglia: parallel and integrative networks J. Chem. Neuroanat. 26317-30

[57] Deffains M, Iskhakova L, Katabi S, Haber S N, Israel Z and Bergman H 2016 Subthalamic, not striatal, activity correlates with basal ganglia downstream activity in normal and parkinsonian monkeys Elife 5 el6443
[58] Meidahl A C, Tinkhauser G, Herz D M, Cagnan H, Debarros J and Brown P 2017 Adaptive deep brain stimulation for movement disorders: the long road to clinical therapy Mov. Disord. 32 810-9

[59] Lanciego J L, Luquin N and Obeso J A 2012 Functional neuroanatomy of the basal ganglia Cold Spring Harb. Perspect. Med. 2 a009621

[60] Sun Y, Wong A K C and Kamel M S 2009 Classification of imbalanced data: a review Int. J. Pattern Recognit. Artif. Intell. $23687-719$

[61] Onaran I, Ince N F, Cetin A E and Abosch A 2011 A hybrid SVM/HMM based system for the state detection of individual finger movements from multichannel ECoG signals 2011 5th Int. IEEE/EMBS Conf. Neural Eng. (IEEE) pp 457-60

[62] Xie Z, Schwartz O and Prasad A 2018 Decoding of finger trajectory from ECoG using deep learning J. Neural Eng. 15036009

[63] Gers F A and Schmidhuber E 2001 LSTM recurrent networks learn simple context-free and context-sensitive languages IEEE Trans. Neural Netw. 12 1333-40

[64] Gers F A, Schmidhuber J and Cummins F 2000 Learning to forget: continual prediction with LSTM Neural Comput. 12 2451-71

[65] Ewert S et al 2018 Toward defining deep brain stimulation targets in MNI space: a subcortical atlas based on multimodal MRI, histology and structural connectivity NeuroImage $170271-82$ 
PhD Thesis, Dan Valsky

III. Determining the true discharge rate and pattern of the striatal projection neurons in Parkinson's disease and dystonia

a. Valsky, D., Heiman Grosberg, S., Israel, Z., Boraud, T., Bergman, H., \& Deffains, M. (2020). What is the true discharge rate and pattern of the striatal projection neurons in Parkinson's disease and dystonia?. eLife, 9, e57445. Advance online publication. https://doi.org/10.7554/eLife.57445 


\title{
What is the true discharge rate and pattern of the striatal projection neurons in Parkinson's disease and Dystonia?
}

\author{
Dan Valsky 1,2, Shai Heiman Grosberg1, Zvi Israel ${ }^{3}$, Thomas Boraud ${ }^{4,5,6}$, \\ Hagai Bergman ${ }^{1,2,3}$, Marc Deffains ${ }^{4,5 *}$
}

${ }^{1}$ Department of Medical Neurobiology, Institute of Medical Research Israel Canada (IMRIC), The Hebrew University - Hadassah Medical School, Jerusalem, Israel; ${ }^{2}$ The Edmond and Lily Safra Center for Brain Sciences, The Hebrew University, Jerusalem, Israel; ${ }^{3}$ Department of Neurosurgery, Hadassah University Hospital, Jerusalem, Israel; "University of Bordeaux, UMR 5293, IMN, Bordeaux, France; ${ }^{5} \mathrm{CNRS}$, UMR 5293, IMN, Bordeaux, France; ${ }^{6} \mathrm{CHU}$ de Bordeaux, IMN Clinique, Bordeaux, France

\begin{abstract}
Dopamine and striatal dysfunctions play a key role in the pathophysiology of Parkinson's disease (PD) and Dystonia, but our understanding of the changes in the discharge rate and pattern of striatal projection neurons (SPNs) remains limited. Here, we recorded and examined multi-unit signals from the striatum of $P D$ and dystonic patients undergoing deep brain stimulation surgeries. Contrary to earlier human findings, we found no drastic changes in the spontaneous discharge of the well-isolated and stationary SPNs of the PD patients compared to the dystonic patients or to the normal levels of striatal activity reported in healthy animals. Moreover, cluster analysis using SPN discharge properties did not characterize two well-separated SPN subpopulations, indicating no SPN subpopulation-specific (D1 or D2 SPNs) discharge alterations in the pathological state. Our results imply that small to moderate changes in spontaneous SPN discharge related to PD and Dystonia are likely amplified by basal ganglia downstream structures.
\end{abstract}

*For correspondence:

marc.deffains@u-bordeaux.fr

Competing interests: The authors declare that no competing interests exist.

Funding: See page 22

Received: 31 March 2020

Accepted: 14 August 2020

Published: 19 August 2020

Reviewing editor: Aryn $\mathrm{H}$ Gittis, Carnegie Mellon University, United States

(c) Copyright Valsky et al. This article is distributed under the terms of the Creative Commons Attribution License, which permits unrestricted use and redistribution provided that the original author and source are credited.

\section{Introduction}

Parkinson's disease (PD) and Dystonia are two of the most common movement disorders, and have a wide spectrum of etiologies and clinical presentations. To date, the pathophysiology of PD and Dystonia is still debated. Traditionally, PD is attributed to the degeneration of the midbrain dopaminergic neurons which also disrupts other neuromodulary systems, including the cholinergic, serotoninergic and histaminergic systems (Bolam and Ellender, 2016; Deffains and Bergman, 2015; Fox et al., 2009). Although there is no degeneration of the midbrain dopaminergic neurons in Dystonia, an imbalance between the midbrain dopaminergic and striatal cholinergic systems (Aosaki et al., 2010; Benarroch, 2012; Bonsi et al., 2011; Pisani et al., 2007), as well as cerebellum dysfunction (Fremont et al., 2017; Helmich, 2018; Helmich et al., 2012; Tewari et al., 2017; Wu and Hallett, 2013) are present in both PD and Dystonia. Nevertheless, basal ganglia (BG) dysfunction is still regarded as the source of the cardinal symptoms of both diseases (Mink, 1996; Wichmann, 2018) and deep brain stimulation (DBS) in the BG (subthalamic nucleus, STN and internal segment of the globus pallidus, GPi) is an effective invasive treatment for both diseases (Limousin et al., 1998; Moro et al., 2017; Odekerken et al., 2016; Ostrem et al., 2017).

The striatum (i.e., the main input structure of the BG network) is the main recipient of midbrain dopaminergic neurons (Menegas et al., 2015) and also receives di-synaptic cerebellar projections 
(Bostan et al., 2013; Hoshi et al., 2005). Most [ 95\% and $70-80 \%$ in rodents and primates, respectively (Graveland et al., 1985; Petryszyn et al., 2018; Petryszyn et al., 2014)] striatal neurons are medium spiny projection neurons (SPNs) that receive afferents from the cortex and the thalamus, and together with the STN neurons, innervate the central (i.e., external segment of the globus pallidus, GPe) and output (i.e., GPi and substantia nigra reticulata, SNr) BG structures (Albin et al., 1989; Gerfen et al., 1990). Therefore, alterations in striatal signaling disrupt normal BG activity and may lead to the manifestation of the motor and non-motor symptoms of PD and Dystonia (Albin et al., 1989; Bergman et al., 1990; Gerfen et al., 1990).

Dysregulation of BG activity may consist of changes in discharge rate (Albin et al., 1989; Gerfen et al., 1990). Unlike in all other BG structures, extracellular recordings of spiking activity in non-human primates (NHPs) reveal that SPNs have a very low discharge rate $(\sim 1-2 \mathrm{~Hz}$ at rest) and are phasically active (i.e. emit short bursts) around relevant behavioral events (Crutcher and DeLong, 1984; Deffains et al., 2010; Kimura et al., 1990). However, previous studies of the BG in the NHP model of PD have mainly focused on the STN, GPe and GPi which are structures with a high frequency tonic discharge (i.e. 25-70 Hz at rest) (Deffains et al., 2016; Filion and Tremblay, 1991; Wichmann and DeLong, 2003). These studies have reported excessive GPi/SNr discharge rates in PD that lead to an increase in BG inhibitory outputs to the thalamus and the frontal cortex motor areas (Wichmann and DeLong, 2003). Conversely, it is assumed that Dystonia (and other hyper-kinetic states) are characterized by reduced GPi/SNr activity (Guehl et al., 2009).

More recent studies of the BG in animal (rodent and NHP) models of PD and human patients (undergoing DBS procedures) have dealt with changes in discharge patterns and synchronization. Parkinsonism-related $\beta$ oscillations have been observed in local field potentials (LFPs) recorded in all BG structures, including the striatum (Deffains et al., 2016; Kondabolu et al., 2016; Lemaire et al., 2012; Singh and Papa, 2019). Similarly, low frequency (4-12 Hz) LFP oscillations have been recorded in the BG network of dystonic patients (Piña-Fuentes et al., 2018; Silberstein et al., 2003). Finally, synchronous $\beta$ oscillations are commonly observed in the spiking activity of the STN, GPe and GPi of MPTP-treated monkeys (Deffains et al., 2018; Deffains et al., 2016; Soares et al., 2004) and PD patients (Brown, 2003; Kuhn et al., 2005; Moshel et al., 2013; Zaidel et al., 2010).

Nevertheless, direct evidence of abnormal activity of the striatal SPNs is still elusive. Previous studies utilizing the NHP model of PD reported striking increases ( 15 fold increase from the normal discharge rate of $\sim 1-2 \mathrm{~Hz}$ ) in the firing rate of the SPNs subsequent to striatal dopamine depletion and the induction of parkinsonism (Liang et al., 2008; Singh et al., 2016). The same research group also reported a high discharge rate of SPNs recorded in PD and dystonic patients $(\sim 30 \mathrm{~Hz}$ and $9 \mathrm{~Hz}$, respectively) (Singh et al., 2016). They also found a significant change in the firing pattern of striatal neurons, with many SPNs exhibiting bursting activity in PD patients and MPTP monkeys as compared to a smaller fraction in patients with Dystonia (Singh et al., 2016). Finally, the SPNs of patients with an essential tremor (ET, regarded as a non-BG disorder) have a very low discharge rate $(\sim 2 \mathrm{~Hz})$ and no tendency to burst, as reported in normal NHPs (Liang et al., 2008; Singh et al., 2016; Singh et al., 2015).

These spectacular changes in the discharge rate and pattern of SPNs in the NHP model of PD run counter results obtained in our research group. We recorded the activity of SPNs and other BG neurons in Vervet monkeys before and after systemic 1-methyl-4-phenyl-1,2,3,6-tetrahydropyridine (MPTP) treatment and the induction of severe parkinsonian symptoms. Although we found robust changes in the discharge properties (rate, pattern and synchronization) of the STN, GPe and BG output structures, we did not observe any difference in the discharge rate $(\sim 2-3$ $\mathrm{Hz}$ ) or pattern of SPNs in the MPTP-treated monkeys in comparison to the recordings in the same monkeys before MPTP treatment (Deffains et al., 2018; Deffains et al., 2016; Deffains and Bergman, 2019).

Extracellular recordings of SPN spiking activity of anesthetized (Chen et al., 2006; Mallet et al., 2006; Sharott et al., 2017) and awake (Chen et al., 2006; Kish et al., 1999) rats before and after striatal dopamine depletion by 6-hydroxydopamine (6-OHDA) treatment have revealed a significant, but very slight increase in the SPN discharge rate. Rodent studies make it possible to differentiate between SPNs expressing D1 and D2 dopamine receptors. A significant imbalance (but see Ketzef et al., 2017) in the discharge rate and calcium dynamics of D1 and D2 SPNs was observed in the dopamine-depleted striatum (Mallet et al., 2006; Parker et al., 2018; Sharott et al., 2017). In particular, D2 SPNs increased their discharge (Mallet et al., 2006; Sharott et al., 2017) and were 
also prone to being entrained to parkinsonian $\beta$ oscillations (Sharott et al., 2017). Nevertheless, the absolute increase in the discharge rate even of the D2 SPNs was still modest (from $\sim 0.5$ to $2.8 \mathrm{~Hz}$ ) (Sharott et al., 2017). Moreover, recent studies have reported no significant increase in the low discharge rate of either SPN subpopulation in striatal dopamine-depleted mice (Ketzef et al., 2017; Maltese et al., 2019).

Since only one research group has recorded drastic increases in the spontaneous spiking activity in the striatum of PD patients in comparison to either dystonic patients or the normal levels of striatal activity reported in healthy animals (Singh et al., 2016), we examined our human patient data to determine whether we could report similar changes. Our study goes beyond previous reports (Singh et al., 2016) by utilizing a machine learning algorithm to automatically detect striato-pallidal border, and applying objective methods for the identification of units and the quantification of their isolation quality and stationarity. Although extracellular recording methods cannot discriminate between the spiking activity of striatal D1 and D2 SPNs, we assumed that if only one population of striatal neurons was strongly affected by the pathological state, we should observe a significantly higher discharge rate of SPNs than that reported in normal NHPs and ET patients, and/or distinct clusters of SPN activity in our patients. Using this data-driven automated approach, we carefully examined spontaneous single-unit activity from the posterior putamen (i.e., the sensorimotor domain of the striatum) of both PD and dystonic patients undergoing GPi-DBS surgeries and compared their discharge rates and patterns.

\section{Results}

\section{Database and spike sorting results}

In this study, 93 microelectrode trajectories (48 in PD and 45 in Dystonia) were used, yielding a total of 933 and 718 microelectrode recording (MER) segments within the posterior putamen (i.e., posterior to the anterior commissure, Figure 1 and Video 1) of patients suffering from PD and Dystonia, respectively. Microelectrode trajectories from patients suffering from non-genetic $(\mathrm{N}=27)$ and genetic ( $N=18$ ) Dystonia were pooled, since the results did not differ statistically. Applying automatic spike sorting on these MER segments identified 5237 units (2917 in PD and 2320 in Dystonia). The isolation score (Joshua et al., 2007) - ranging from 0 (i.e., highly noisy) to 1 (i.e., perfect isolation) - was calculated for each of these units. Moreover, since injury potentials are often observed during extracellular microelectrode recordings of striatal activity (DeLong, 1971), the stationarity of the firing rate and spike amplitude of the well-isolated units (isolation score $\geq 0.6$ ) was assessed.

All surgeries were carried out while the patients were fully awake (no sedation or anesthesia) and the PD patients were off dopaminergic medication (overnight washout $>12 \mathrm{hr}$ ). The DBS target was the ventro-posterior-lateral portion of the GPi for all patients, and trajectory angles were only slightly modified according to patient's anatomy. To graphically illustrate the recording locations in the striatum, we employed visualization of group-based microelectrode track trajectories in both PD and Dystonia (Figure 1, see also Video 1). All recordings were regularly sampled in space within the striatum (see Materials and methods). All patients provided their written informed consent and the study was approved by the Institutional Review Board of Hadassah Hospital in accordance with the Helsinki Declaration (reference code: 0168-10-HMO).

Figure 2A depicts examples of the striatal spiking activity recordings (left and middle). The right plots display the superimposed waveforms of the extracellular action potentials of the sorted units. The units are ordered as a function of their isolation score (Joshua et al., 2007). The median and mean discharge rates of all the recorded units with an isolation score greater than or equal to one of the 10 evenly spaced values between 0 and 0.9 were calculated (Figure 2B). A significant negative correlation between neuronal discharge rate and isolation score was found in the striatum of the PD (Pearson's $r=-0.87$ and -0.89 for median and mean discharge rate, respectively, $p<0.01$, Figure 2B) and dystonic (Pearson's $r=-0.89$ and -0.85 for median and mean discharge rate, respectively, $p<0.01$, Figure $2 B$ ) patients, indicating that the units with the lowest isolation score had the highest discharge rates. 


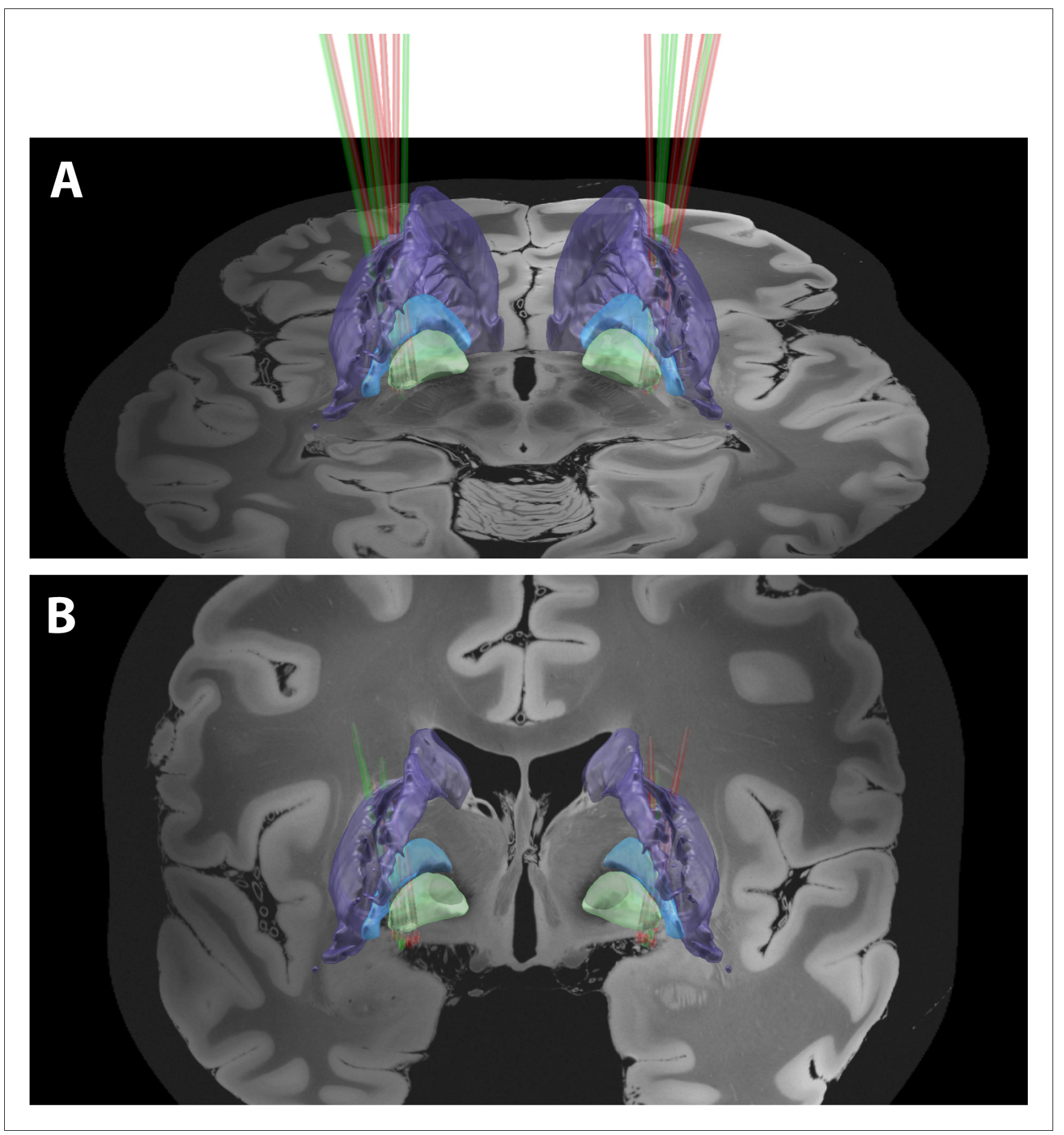

Figure 1. A $3 D$ rendering of group-based microelectrode track trajectories in the posterior putamen. (A) Posterior view of the microelectrode track trajectories from both PD and dystonic patients. An axial image from the normalized scan, at the level of the rostral midbrain, is shown as a backdrop. The image was acquired on a 7T MRI scanner with a $100 \mu \mathrm{m}$ T1 scan of an ex-vivo human brain. The definition of the striatum (purple), GPe (light blue) and GPi (light green) boundaries are defined by the DISTAL atlas. (B) Same as (A) but with a coronal image. A total of 36 microelectrode track trajectories, 13 in PD (green) and 23 in Dystonia (red), are visualized for four PD and six dystonic patients. 


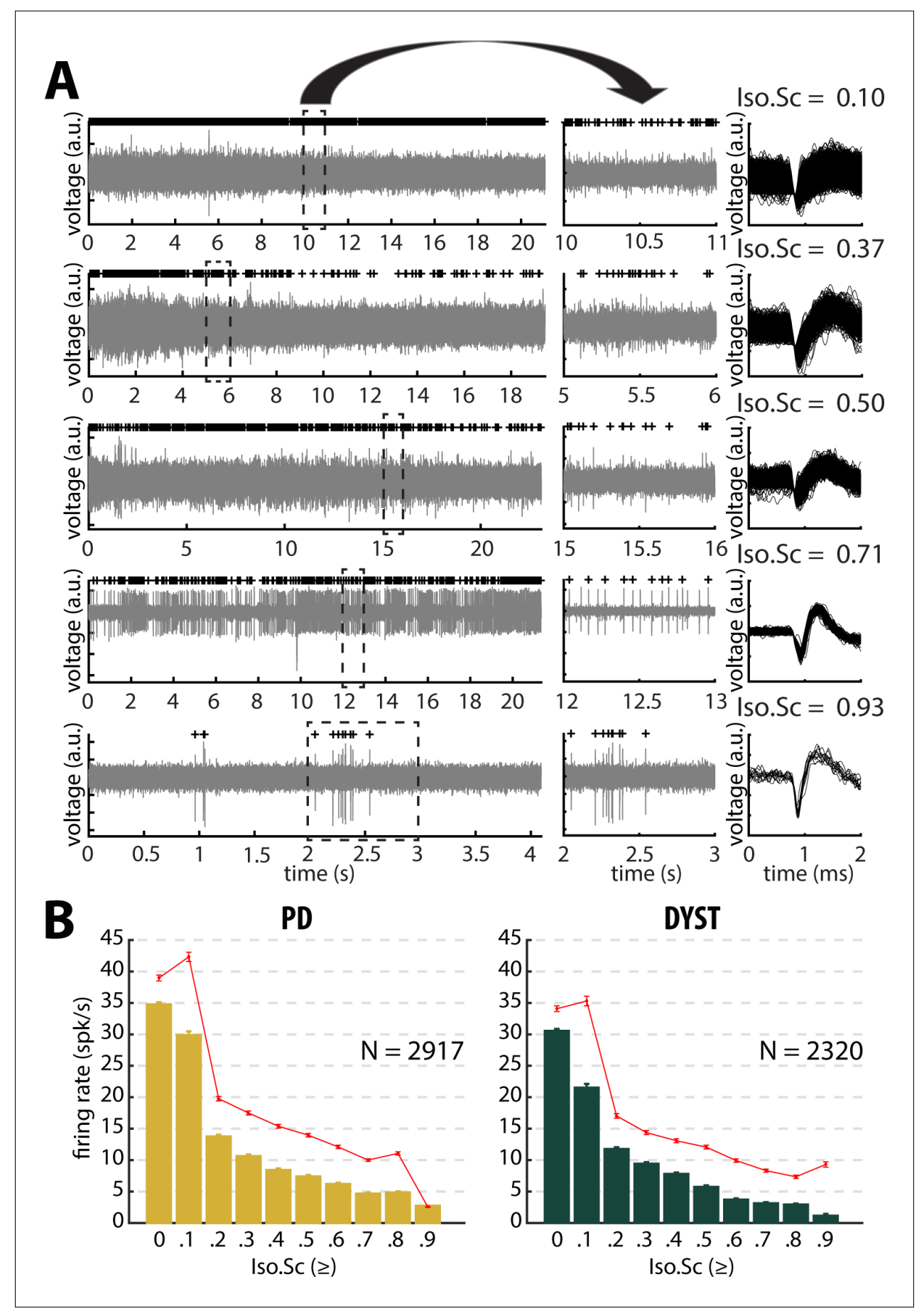

Figure 2. Striatal firing rate in parkinsonian (PD) and dystonic (DYST) patients decreases as isolation quality of the units increases. (A) Examples of fulllength (left panels) and $1 \mathrm{~s}$ (middle panels) striatal spiking recordings. Above the spiking activity is the digital display of the detection (spike train) of the sorted unit from the recording. Spike waveforms are superimposed (right panels). Isolation score (Iso.Sc) indicates the isolation quality of the sorted unit. (B) Evolution of the firing rate as a function of the isolation score. Each bar indicates the median firing rate of the sorted units with an isolation score greater than or equal to a certain value (i.e., the bin labelled 0.8 contains all the units with an isolation score $\geq 0.8$ ). Error bars represent MADs (i. e., median absolute deviations). Means \pm SEMs (i.e., standard errors of the mean) are shown in red. $\mathrm{N}$ is the number of sorted units. The online version of this article includes the following source data for figure 2 :

Source data 1. Firing rate and isolation score of all units in PD.

Source data 2. Firing rate and isolation score of all units in Dystonia. 


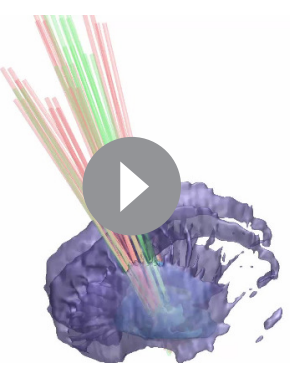

Video 1. 3D rotating view of group-based microelectrode trajectories.

https://elifesciences.org/articles/57445\#video1

\section{No drastic increase in the firing rate of the striatal neurons of PD and dystonic patients}

The median firing rate of all the sorted striatal units was relatively high $(-30-40 \mathrm{~Hz})$ in both diseases (Figure 3, first column) and consistent (at least for PD) with the values reported by Singh et al., 2016. Similarly, the firing rate of the dystonic patients was significantly lower than the PD firing rate (Mann-Whitney U-test, $p<0.001$, Figure 3-figure supplement 1, first column). Nevertheless, this significant difference disappeared and the striatal firing rate decreased dramatically when only comparing the firing rate of the well-isolated units (isolation score $\geq 0.6$ ) (Fig-

ure 3 and Figure 3-figure supplement 1, first column). Hence, there was no drastic change in the striatal firing rate of the well-isolated units of the PD and dystonic patients.

Inspection of the spike train of the well-isolated units (isolation score $\geq 0.6$ ) revealed that although the units had been graded as high-isolation score units, their spike train could be non-stationary (Figure 4). To assess the stationarity of the spike trains, we examined the temporal (linear) evolution of their firing rates and spike amplitudes (see Materials and methods). Figure 4A shows three representative examples of well-isolated non-stationary units (left panels) and three representative examples of well-isolated stationary units (right panels) from the striatum of PD patients. Here, we used the slopes of the linear regression lines (reflecting the temporal evolution of the firing rate and spike amplitude of the well-isolated units) to define stationary units (Figure $4 B$ and C). Interestingly, the firing rate slope values and spike amplitude slope values of the well-isolated units were uniformly distributed, thus indicating that these two spiking features were independent in both PD and Dystonia (Figure 4D and E). This analysis also showed that the non-stationary units (i.e., data points outside the shaded areas) did not necessarily exhibit both a non-stationary firing rate and a nonstationary spike amplitude (Figure 4D and E). For both PD and Dystonia, the firing rate of the stationary units was significantly lower than the firing rate of the non-stationary units (Mann-Whitney U-test, $\mathrm{p}<0.01$ and 0.001 for PD and dystonic patients, respectively, Figure 4F). Thus, inclusion of non-stationary units could have erroneously inflated the striatal firing rate in PD and dystonic patients. Removing the non-stationary units reduced the striatal firing rate of the well-isolated units in the striatum of both PD and dystonic patients (Figure 3 and Figure 3-figure supplement 1, first column). Moreover, the striatal firing rate remained similar between both diseases after this maneuver.

Equally important, the mean values of the firing rate (Figure 3-figure supplement 1, first column) were systematically higher than the median values (Figure 3, first column), suggesting that whatever the quality of the isolation and the stationarity of the striatal units, the distributions of the firing rate were not normal and were skewed to the right. Figure 5 depicts the distributions of the firing rate of all and only the well-isolated units (Figure 5A and B, respectively). For both PD and Dystonia, and for all levels of spike isolation, the distributions of the firing rate of the SPNs were not normally distributed, but rather were strongly skewed to the right (Figure 5). We therefore used the median (Figure 3) rather than the mean (Figure 3-figure supplement 1) to represent the central moment of the distributions of the discharge properties (including the discharge rate) of the SPNs. The median \pm MAD (median absolute deviation) of the discharge rate was $3.85 \pm 1.18 \mathrm{~Hz}$ and $2.55 \pm 0.81 \mathrm{~Hz}$ for the well-isolated stationary SPNs in PD and Dystonia respectively - i.e., in the same range as reported for the controls in animal studies and with no significant difference between PD and Dystonia (Mann-Whitney U-test, $p>0.05$, Figure 4F).

\section{No evidence for bursty patterns in the striatal spiking activity of PD and dystonic patients}

To characterize the pattern of the spike trains (i.e., irregular, periodic or bursty) and compare them between PD and dystonic patients, we examined the time interval histograms (TIHs) of the inter- 


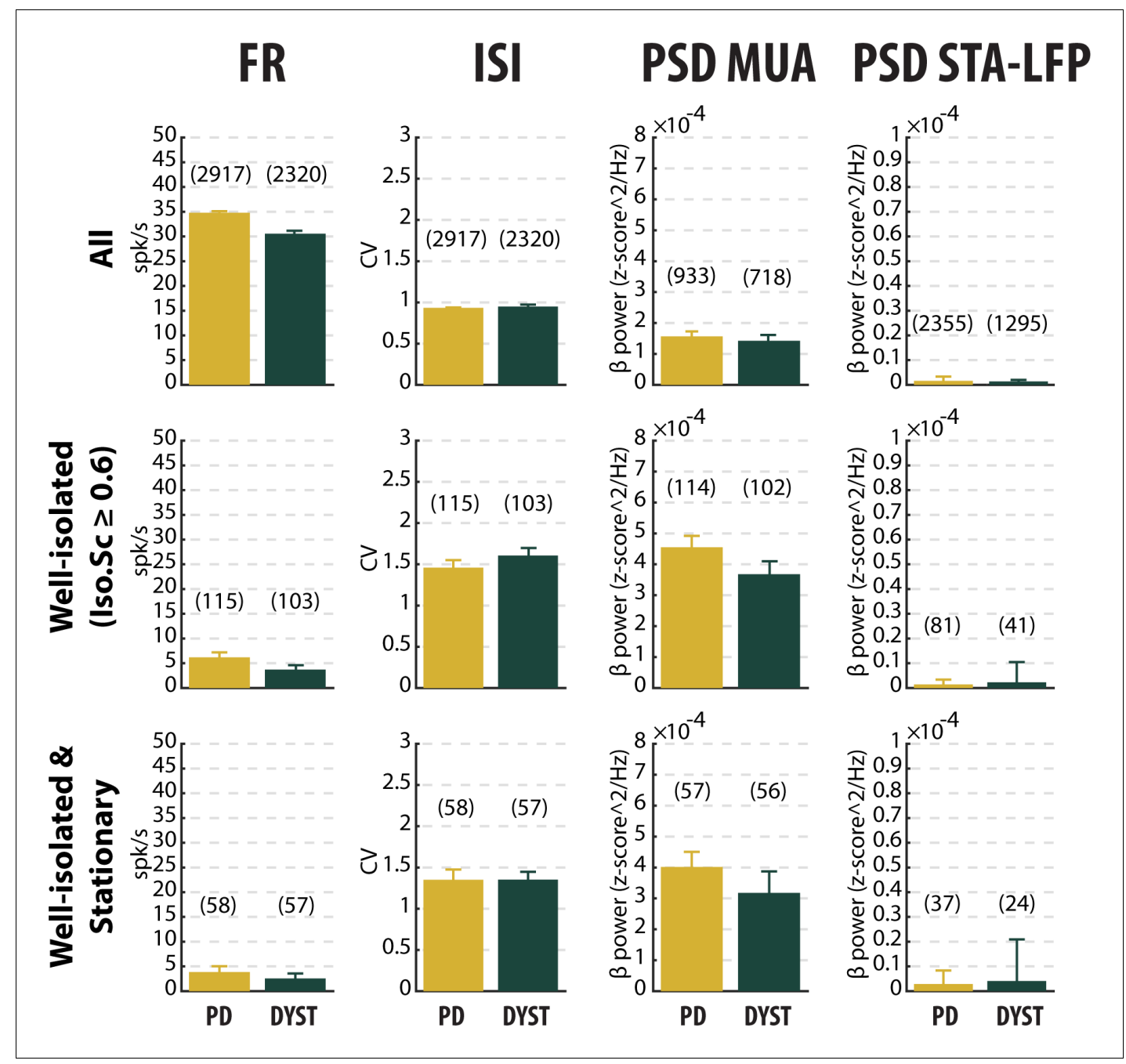

Figure 3. No drastic or specific change in the features (median \pm MAD values) of striatal neuronal activity in parkinsonian (PD) and dystonic (DYST) patients. Comparison of the firing rate (first column), the coefficient of variation (CV) of the inter-spike interval (ISI) (second column), the $\beta$ power of the multi-unit activity (MUA, spiking activity) recorded in the vicinity of a sorted unit (third column) and the $\beta$ power of the spike-triggered average (STA) of the local field potential (LFP) (fourth column) when considering all (upper panels), only the well-isolated (middle panels) and only the well-isolated stationary (lower panels) units. Since each signal was Z-score normalized (using its mean and SD) prior to PSD calculations, the $\beta$ power of both the MUA (third column) and STA-LFP (fourth column) are expressed in z-scoré2/Hz. Each bar indicates the median value and error bars represent MADs. Same y-axis scales as in Figure 3-figure supplement 1 and Figure 3-figure supplement 5. Numbers in parentheses indicate the sizes of the samples. Note that the sizes of the samples differ between the columns because (i) the number of sorted units from each MUA ranged from 1 to 5 and (ii) the LFPs were not systematically recorded.

The online version of this article includes the following source data and figure supplement(s) for figure 3:

Source data 1. Spiking features of all units in PD.

Source data 2. Spiking features of the well-isolated units in PD.

Source data 3. Spiking features of the well-isolated and stationary units in PD.

Source data 4. Spiking features of all units in Dystonia.

Source data 5. Spiking features of the well-isolated units in Dystonia.

Source data 6. Spiking features of the well-isolated and stationary units in Dystonia.

Figure supplement 1. No drastic or specific change in the features (mean $\pm \mathrm{SEM}$ values) of striatal neuronal activity in parkinsonian (PD) and dystonic (DYST) patients.

Figure supplement 2. Comparison of the spiking features between the putative well-isolated and stationary SPNs and TANs in PD patients.

Figure supplement 3. Comparison of the spiking features between the putative well-isolated and stationary SPNs and TANs in dystonic patients. Figure supplement 4. Disease effects on the outliers of the different features of striatal neuronal activity.

Figure supplement 5. Median (upper panels) and mean (lower panels) values of the main features of striatal neuronal activity in parkinsonian (PD) and dystonic (DYST) patients. 
A

- non-stationary unit \#1

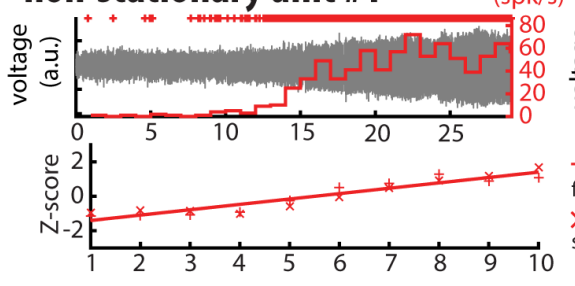

- non-stationary unit \#2
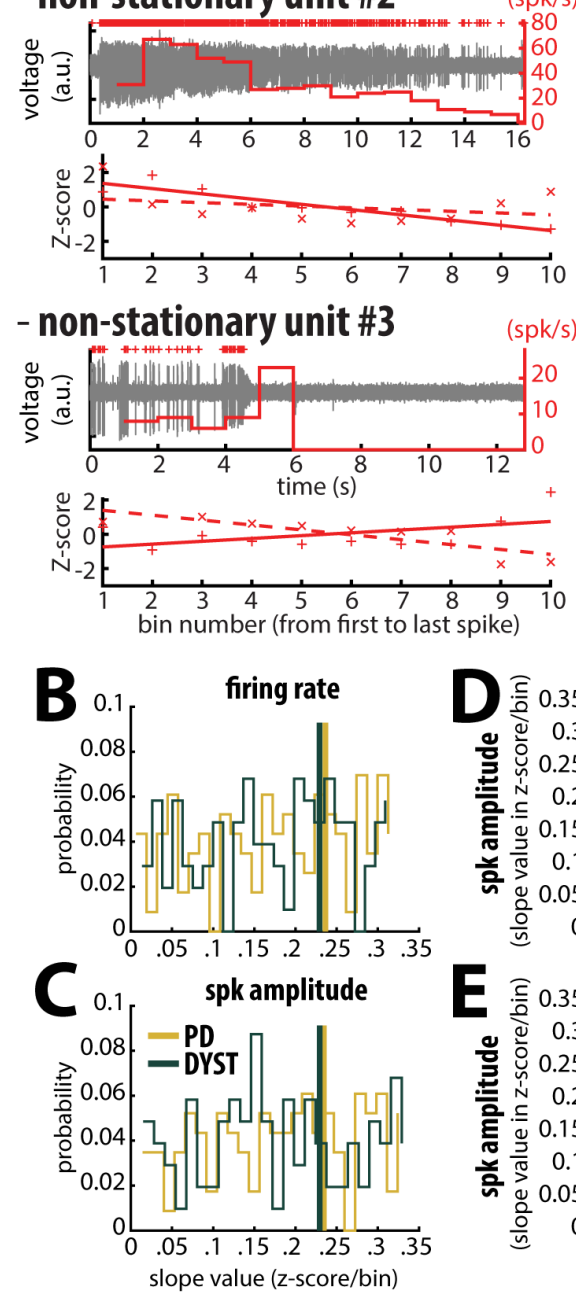

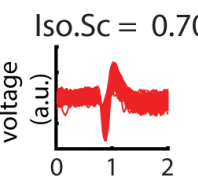

$+-$

firing rate $(m=0.3122)$

$\times--$
spk amplitude $(m=0.3133)$
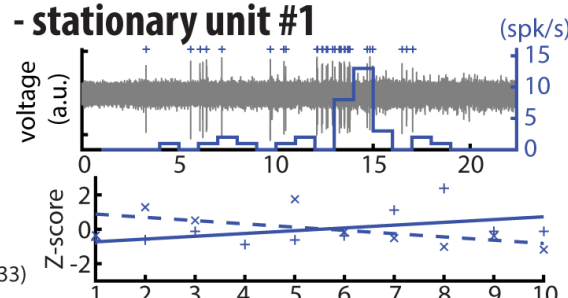

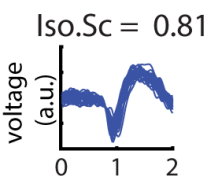

$+-$

firing rate $(m=0.1618)$

$x$ - -

spk amplitude $(m=0.1897)$

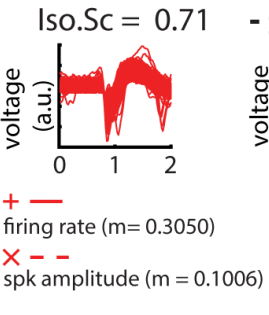

- stationary unit \#2

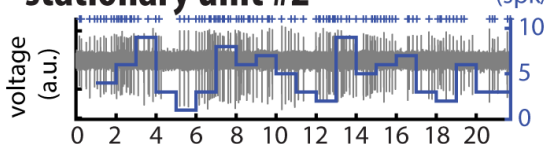

ㄴ.

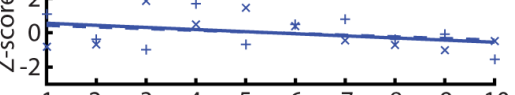

$\begin{array}{lllllllllll}1 & 2 & 3 & 4 & 5 & 6 & 7 & 8 & 9 & 10\end{array}$

\section{stationary unit \#3}

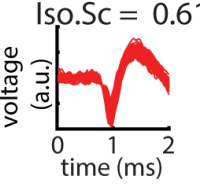

+ -

firing rate $(m=0.1645)$

$x--$

spk amplitude ( $m=0.2848$ )

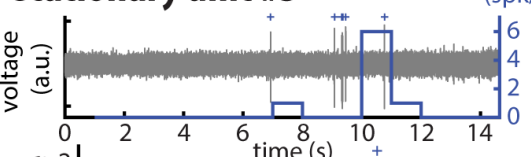

离 2

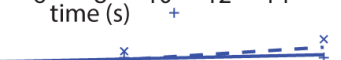

/s) Iso.Sc $=0.79$

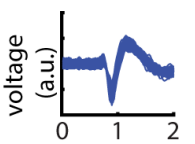

$+-$

firing rate $(m=0.1230)$

X - -

spk amplitude $(m=0.0953)$

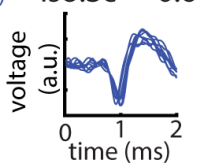

+ -

firing rate $(m=0.0626)$

$x--$

spk amplitude $(m=0.1793)$
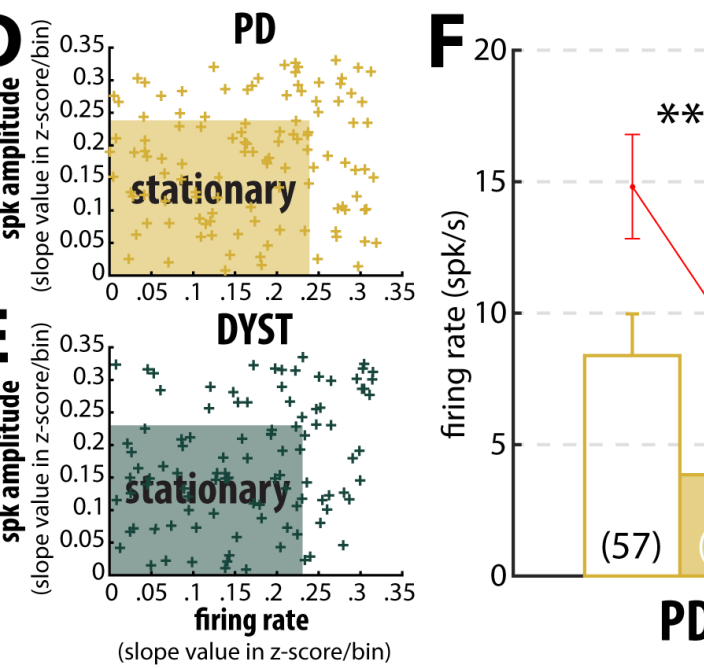

NS

bin number (from first to last spike)

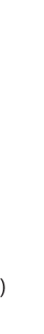

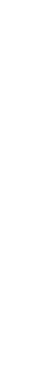


Figure 4 continued

or equal to the 70th percentile were defined as non-stationary units. (D-E) Scatter plots showing the firing rate slope values and spike amplitude slope values of the well-isolated (non-stationary and stationary) units recorded in the striatum of PD and dystonic patients. Shaded areas represent the zones of the stationary units which are delineated by the 70th percentile of both the slope values and spike amplitude slope values. (F) Comparison of the firing rate of the non-stationary and stationary units. Each bar indicates the median firing rate of the sorted units. Error bars represent MADs. Means \pm SEMs are shown in red. Numbers in parentheses indicate the numbers of non-stationary and stationary units. ${ }^{* *},{ }^{* * *}$ and NS indicate significant ( $p<0.01$ and 0.001 ) and non-significant differences, respectively (Mann-Whitney U-test).

spike intervals (ISIs). Although weak (Figure 3, second column), there was a significant difference in the coefficient of variation (CV) of the ISls of all the sorted striatal units between the PD and dystonic patients (Mann-Whitney U-test, $p<0.001$, Figure 3 -figure supplement 1, second column). Nevertheless, as was the case for the striatal firing rate, this difference vanished when only considering the well-isolated or well-isolated stationary units (Figure 3 and Figure 3-figure supplement 1, second column). Again, the mean values of the CV of the ISIs (Figure 3-figure supplement 1, second column) were systematically higher than the median values (Figure 3, second column), indicating skewed distributions. Moreover, the distributions of TIHs of the ISIs of the well-isolated stationary units matched the Poisson temporal distribution of the spikes (Figure 6A). Accordingly, the mean autocorrelograms of the spike trains did not reveal any periodic or bursty firing patterns in the

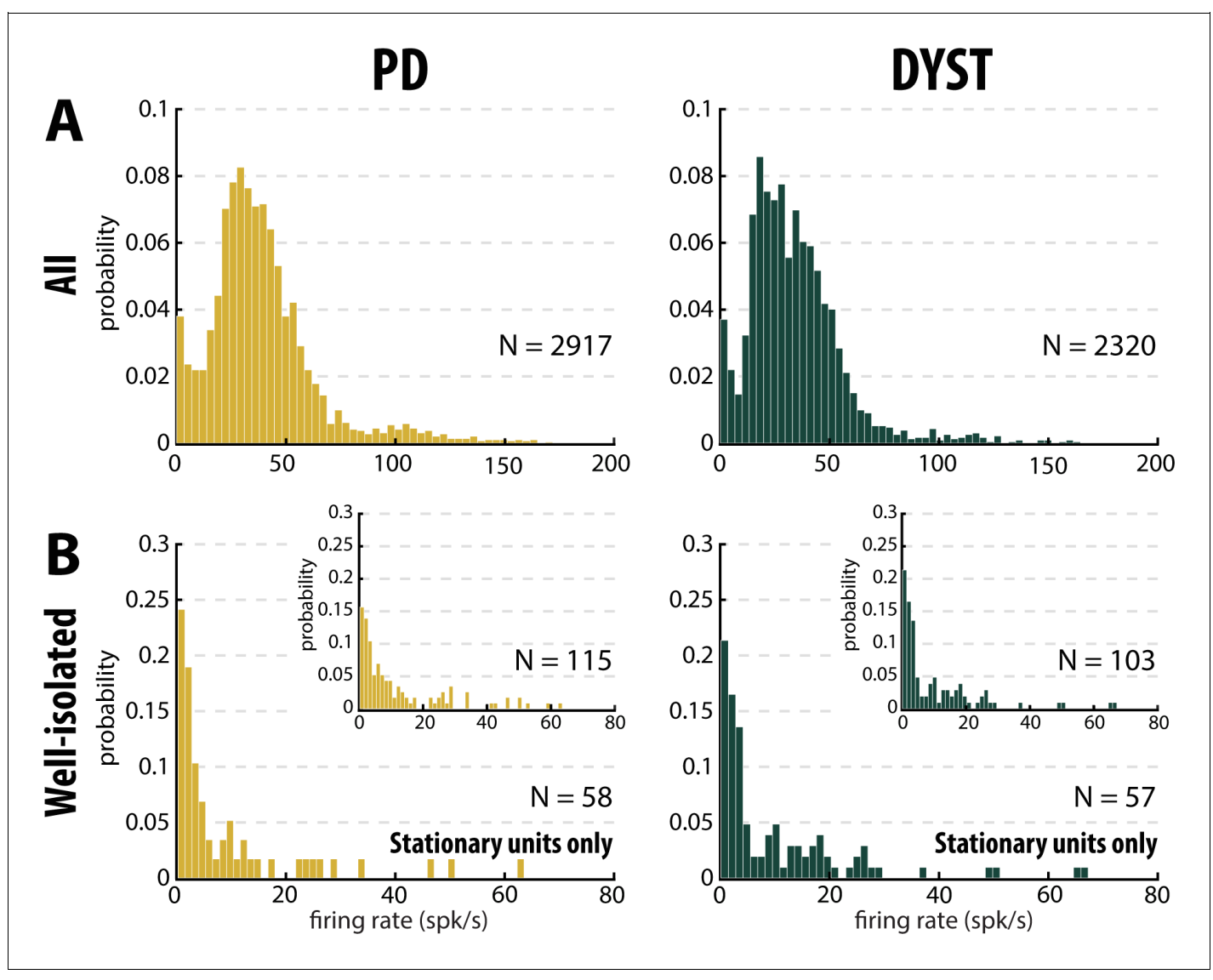

Figure 5. Distributions of the striatal firing rate are skewed to the right in parkinsonian (PD) and dystonic (DYST) patients. (A) Firing rate of all sorted units regardless of their isolation quality. (B) Firing rate of the well-isolated stationary units only. Skewness $=2.34$ and 3.94 for PD and dystonic patients, respectively. Insets: Firing rate of the well-isolated units (non-stationary and stationary units pooled). Skewness $=1.73$ and 2.36 for PD and dystonic patients, respectively. $\mathrm{N}$ is the number of sorted units. 


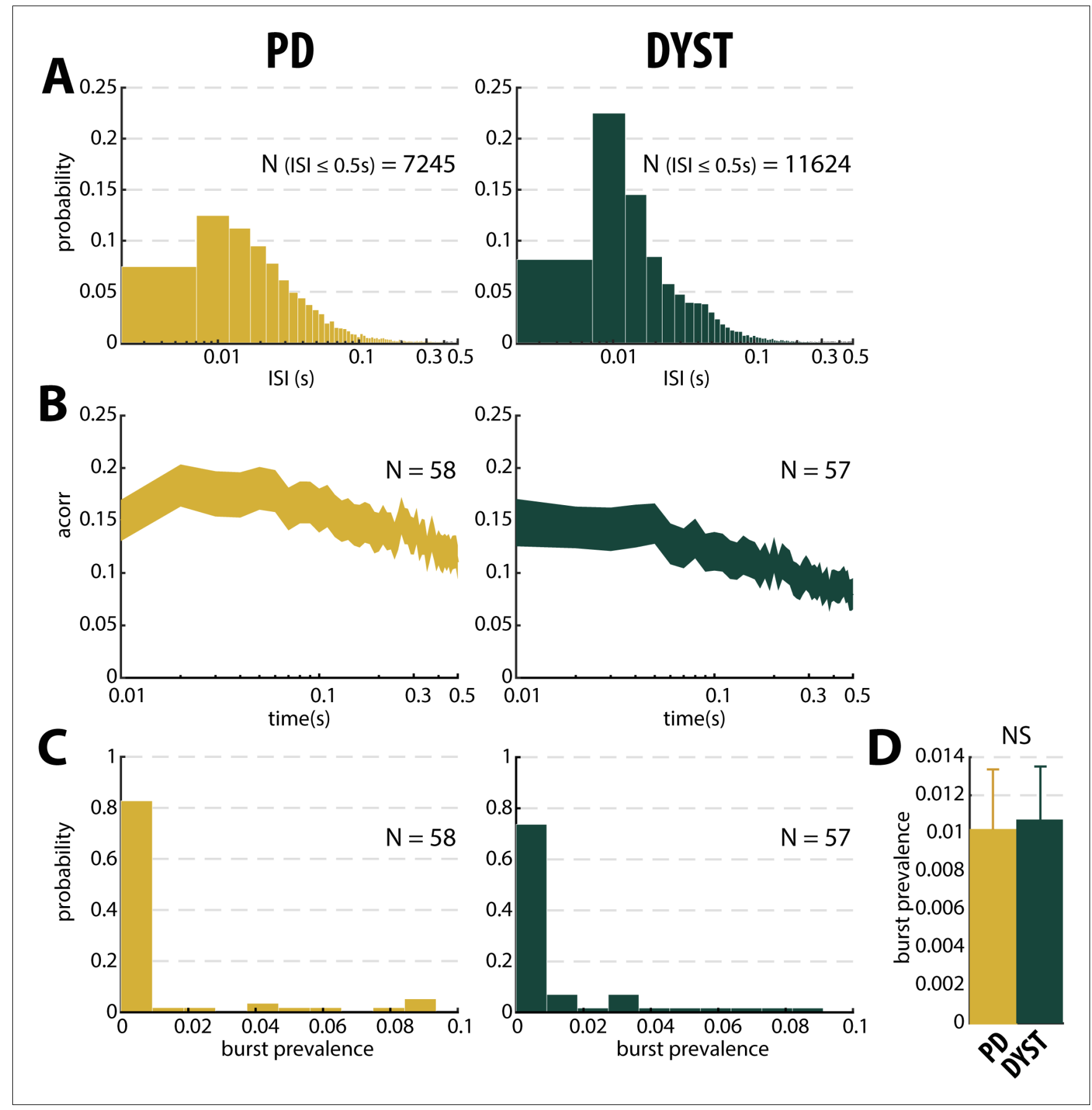

Figure 6. No evidence for burst patterns in the striatal spiking activity of parkinsonian (PD) and dystonic (DYST) patients. (A) Time interval histograms of the inter-spike intervals (ISI) of the well-isolated (isolation score $\geq 0.6$ ) stationary units. For better visualization, 191 and 292 ISIs $>0.5 \mathrm{~s}$ were removed from the PD and DYST striatal units, respectively. Abscissas are in log scale. (B) Average (mean $\pm \mathrm{SEM}$ ) autocorrelograms of the well-isolated stationary units. For each autocorrelogram, values were normalized so that the autocorrelation values ranged from 0 to 1 . Abscissas are in log scale. $\mathrm{N}$ is the number of well-isolated stationary units averaged. (C) Distributions of the values of burst prevalence for the spike train of the well-isolated stationary units. For each unit, episode prevalence represents the probability that the discharge pattern was bursty. (D) Mean values of burst prevalence for the well-isolated stationary units recorded in the striatum of parkinsonian and dystonic patients. Error bars represent SEMs. N is the number of well-isolated stationary units averaged. NS: non-significant (Mann-Whitney U-test).

The online version of this article includes the following figure supplement(s) for figure 6 :

Figure supplement 1. No evidence for burst patterns in the striatal spiking activity of parkinsonian (PD) and dystonic (DYST) patients. 
striatal well-isolated stationary units (Figure 6B). Similarly, the prevalence of bursts [detected using the Poisson surprise method with the surprise maximization (SM) search algorithm (Legéndy and Salcman, 1985), see Materials and methods] was relatively low (Figure 6C) and did not differ significantly between PD and dystonic patients (Mann-Whitney U-test, p>0.05, Figure 6D).

\section{Absence of oscillatory spiking activity in the striatum of PD and dystonic patients}

Theoretical studies have shown that neural oscillations can emerge at the population level in networks of neurons exhibiting an irregular (i.e., non-oscillatory) discharge pattern and a low firing rate (Brunel and Hakim, 2008; Kopell and LeMasson, 1994). To overcome the possible confounding effects of the low discharge rate and spatial under-sampling of the striatal SPNs, we investigated multi-unit oscillatory activity rather than single-unit oscillatory activity. Striatal multi-unit activity (MUA) reflects the spiking activity of an ensemble of striatal neurons around a sorted unit.

Comparison of the power spectral densities (PSDs) of the spiking activities recorded in the striatum of the PD and dystonic patients did not reveal any oscillatory phenomena between 3 and $75 \mathrm{~Hz}$, including the $\beta(13-30 \mathrm{~Hz})$ band (Figure 7A and B). Accordingly, no significant difference in the $\beta$ power of the striatal spiking activities (whatever the quality of the isolation and the stationary of the striatal units) was observed between PD and dystonic patients (Mann-Whitney U-test, $p>0.05$, Figure 3, and Figure 3-figure supplement 1, third column), Moreover, we found no significant increases of the $\beta$ (13-30 Hz) power in the spiking activities recorded in the vicinity of the striatal well-isolated stationary units compared to the linearly interpolated baseline $\beta$ power (Wilcoxon signed rank test, $p>0.05$, Figure $7 C$ ), thus supporting the claim of no oscillatory spiking activity in the striatum of PD and dystonic patients.

\section{No locking between spike and $\beta$ LFP oscillations in the striatum of PD and dystonic patients}

The mean PSD of the mono-polar LFPs recorded in the striatum of PD and dystonic patients (Figure 8A) exhibited (ignoring $50 \mathrm{~Hz}$ artifacts) two and three distinct peaks, respectively (at 11 and $21 \mathrm{~Hz}$ for PD patients and 8,22 and $30 \mathrm{~Hz}$ for dystonic patients, Figure 8A, insets). Therefore, we calculated the spike-triggered averages of the LFPs (STAs LFPs). The $\beta$ power in the LFPs recorded around the time of the spikes of all the striatal sorted units was significantly higher in PD than in dystonic patients (Mann-Whitney U-test, p>0.001, Figure 3 and Figure 3figure supplement 1, fourth column). However, this difference disappeared when using the spikes of the well-isolated (non-stationary and stationary or only stationary) units (Figure 3 and Figure 3-figure supplement 1, fourth column). Thus, the spiking activity of well-isolated stationary units recorded in the striatum of both PD and dystonic patients failed to lock with 13$30 \mathrm{~Hz}$ LFP oscillations (Figure 8B).

\section{TANs are hardly distinguishable from SPNs in our human databases}

In order to differentiate between the SPNs and the striatal cholinergic interneurons, the tonically active neurons (TANs, presumably the cholinergic interneurons) were retrospectively identified by three experts (DV, HB and MD) from the well-isolated and stationary striatal units included in both the PD and Dystonia databases. This identification was based on offline visual inspection of their electrophysiological features. The 3D-classifications of the putative SPNs and TANs, using their firing rate, the CV of their ISI and the score of the first principal component (PC1) of their spike waveform did not clearly discriminate them in either PD or dystonic patients (Figure 3-figure supplement 2A and Figure 3-figure supplement 3A, respectively). The same 3D-classification, but using the score of PC2 or PC3 (instead of PC1) yielded the same qualitative results. Unexpectedly, we found that the averaged spike waveform hardly varied between these two subtypes of striatal units in either PD or dystonic patients (Figure 3figure supplement $2 B$ and Figure 3-figure supplement 3B, respectively). Besides, we did not find significant differences in the firing rate of the putative SPNs and TANs in PD (Figure 3figure supplement $2 C$ and $D$, first column) or dystonic (Figure 3-figure supplement $3 C$ and $D$, first column) patients. However, the CV of the ISI was significantly smaller for the putative 


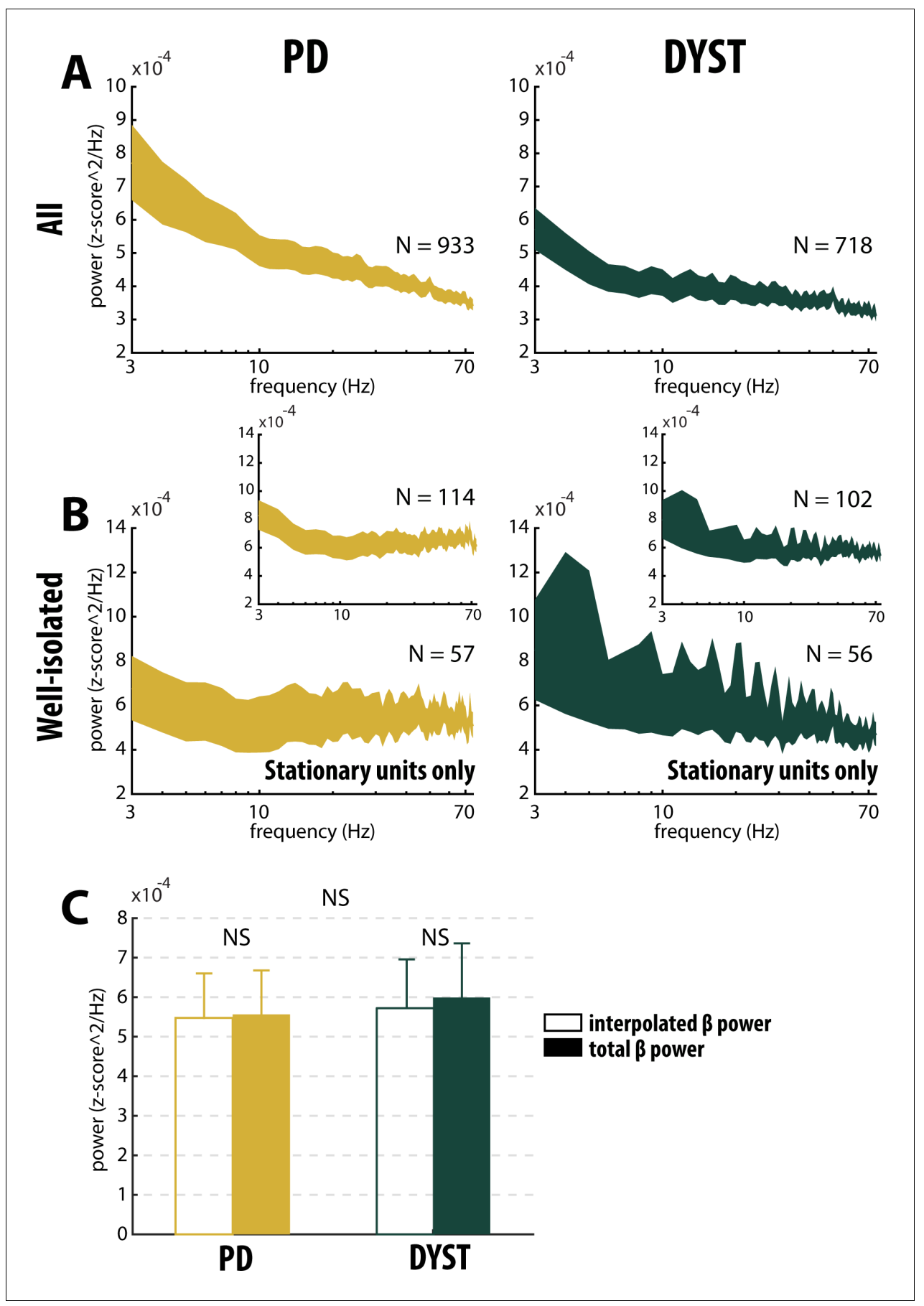

Figure 7. Absence of oscillatory spiking activity in the striatum of parkinsonian (PD) and dystonic (DYST) patients. Average (mean \pm SEM) power spectrum densities (PSDs) of (A) all spiking activities and (B) only spiking activities recorded in the vicinity of well-isolated stationary units. Insets: Average PSDs of the spiking activities recorded in the vicinity of the well-isolated units (non-stationary and stationary units pooled). Since each bandpass filtered spiking signal was Z-score normalized (using its mean and SD) prior to PSD calculations, PSDs are expressed in z-scoré2/Hz. Abscissas are in log scale. $N$ is the number of spiking activities averaged. (C) Average $\beta$ (13-30 Hz) power of the spiking activities recorded in the vicinity of wellisolated stationary units. To interpolate the $\beta$ power, PSDs were linearly interpolated [based on the two closest points that flanked the 13-30 Hz band namely, the values at 12 and $31 \mathrm{~Hz}$ (spectral resolution of $1 \mathrm{~Hz}$ )]. Interpolated $\beta$ power is the mean of the linearly interpolated values between 13 and 30 $\mathrm{Hz}$. Total $\beta$ power is the mean of the observed values between 13 and $30 \mathrm{~Hz}$. Error bars represent SEMs. NS: non-significant (Wilcoxon signed rank test and Mann-Whitney U-test). 


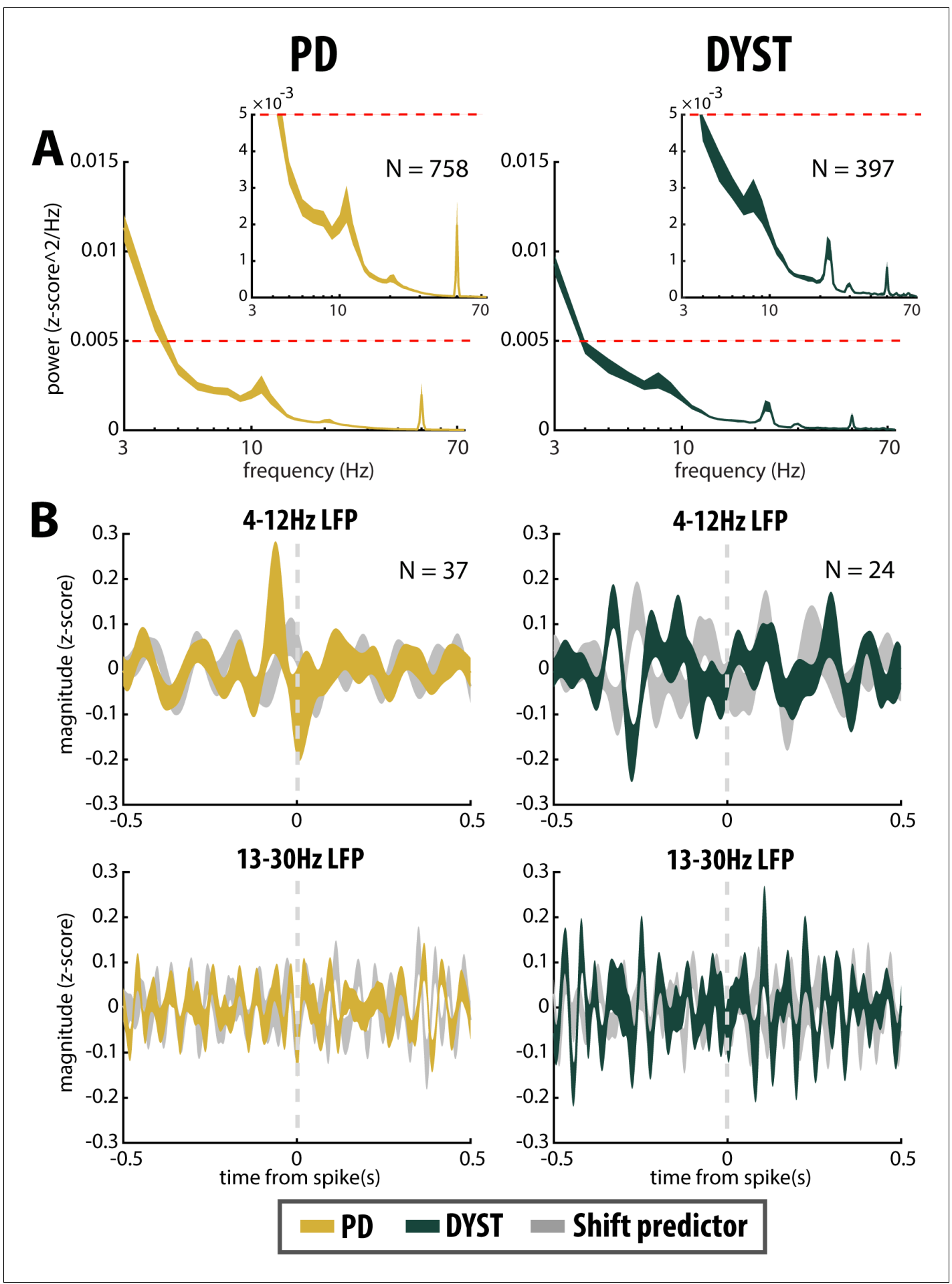

Figure 8. No locking between spike and $\beta$ LFP oscillations in the striatum of parkinsonian (PD) and dystonic (DYST) patients. (A) Average (mean \pm SEM) PSDs of striatal LFPs. In the insets, the ordinates are truncated for better visualization of the power. Abscissas are in log scale. N is the number of LFPs averaged. (B) Population (mean \pm SEM) spike-triggered averages (STAs) of LFP. LFP was recorded in the vicinity of well-isolated stationary units (i.e., spiking activity and LFP were recorded on the same electrode) and offline band-pass filtered from 4 to $12 \mathrm{~Hz}$ (upper panels) or from 13 to $30 \mathrm{~Hz}$ (lower panels). For comparison, STAs-LFP were also calculated after randomly shifting the timestamp of each spike of the spike train [i.e., random time (comprised between 0 and $1 \mathrm{~s}$ ) was added to the timestamp of each spike of the spike train] in order to abolish any relationship between LFP and spiking activity (Shift predictor). Dashed grey vertical lines indicate the time of the spikes (time $=0$ ). N is the number of STAs-LFP averaged. Since each LFP was Z-score normalized (using its mean and SD) prior to PSD and STA calculations, PSDs and magnitudes of the STA-LFPs are expressed in z-scoré2/Hz and z-score, respectively. 
TANs than for the putative SPNs in both diseases (Figure 3-figure supplement 2C and D and Figure 3-figure supplement $3 C$ and $D$, second column), thus corroborating the view that TANs fire in a more regular fashion than the SPNs which are thought to be phasically activated (Crutcher and DeLong, 1984; Deffains et al., 2010; Kimura et al., 1990). The lack of significant differences in the spike waveform and the firing rate between our putative human SPNs and TANs can be accounted in several ways: misclassification of certain neurons, presence of other striatal interneurons (i.e., FSI) and/or differences in extracellular recording techniques (microelectrode type, position/placement, and impedance). Overall, it was difficult to distinguish the TANs from the SPNs in our human databases. Moreover, our spectral analyses did not reveal any significant differences between these two subtypes of striatal neurons in either disease (Figure 3-figure supplement 2C and D, and Figure 3-figure supplement 3C and D, third and fourth columns). Therefore, we considered our sorted striatal spiking activity to reflect SPN spiking activity and neglected the small fraction of TANs (which was probably smaller than our estimation).

\section{Outliers and cluster analysis of the striatal spiking features}

Since we did not have a control condition or baseline levels, we could not identify the significant changes in the spiking features of the striatal neuronal activity. Nevertheless, we searched for outliers (i.e., data points that differ significantly from other observations) of each distribution. We examined the extreme values in the distributions and characterized the data points that could belong to a different population than the rest of the sample set. For each distribution, the values of the spiking feature (i.e., firing rate, CV of ISI, $\beta$ power of the MUA or $\beta$ power of STA-LFP) $\geq 2$ or $\leq-2$ SDs of its mean value were considered outliers (empirical 68-95-99.7 rule). Remarkably, almost all of the outliers corresponded to feature values $\geq 2$ SDs of the mean value, thus indicating (as already observed) that the distributions of the values of the striatal spiking features were skewed to the right in both diseases. Nevertheless, we found that the proportions of outliers (regardless of the spiking feature and the isolation level) were small (Figure 3-figure supplement 4). Moreover, we did not find any significant disease effect on the proportions (chi-square test, $\mathrm{p}>0.05$ ) or the values (Mann-Whitney U-test, $\mathrm{p}>0.05$ ) of these outliers when only considering the well-isolated or well-isolated stationary units (Figure 3-figure supplement 4).

To further test whether two distinct SPN subpopulations could be observed in the current study, we performed 2D k-means cluster analysis with $\mathrm{k}=2$, using the discharge rate and the $\mathrm{CV}$ of the ISIs of each unit as input parameters (Figure 9). The basic idea behind this 2D k-means clustering was to define 2 clusters of data points so that the total within-cluster variation (i.e., the sum of the Squared Euclidean distances from data points to the cluster centroid) would be minimized within each of the two clusters. Using $k=2$ as the predefined number of clusters systematically enforced the separation into two distinct SPN subpopulations. To assess how well-separated the two resulting clusters were, we calculated the silhouette values to measure how close each data point in one cluster was to data points in the other clusters. Silhouette values range from +1 (data points are very distant from other clusters), through 0 (data points are not distinctly in one cluster or another), to -1 (data points are probably assigned to the wrong cluster). The results showed that the two predefined clusters were not well-separated, regardless of the quality of the isolation or the stationarity of the striatal units (Figure 9, insets). In fact, in most of the scenarios, the second cluster contained a few data points with negative silhouette values, and if not, there were not enough data points to consider it a real cluster that represented a distinct and balanced SPN subpopulation. Equally important, in contrast with the view that discharge rate elevation and bursting activity coexist in the striatum of PD patients (Singh et al., 2016), the tendency to burst (CV of the ISIs > 1; Kaneoke and Vitek, 1996) decreased for units with a high discharge rate (Figure 9). Similar 2D or higherorder (3 or 4D) k-means cluster analyses (using the discharge rate, CV of the ISIs, MUA $\beta$ power or STA-LFP $\beta$ power as input parameters) also failed to reveal two well-separated clusters. 


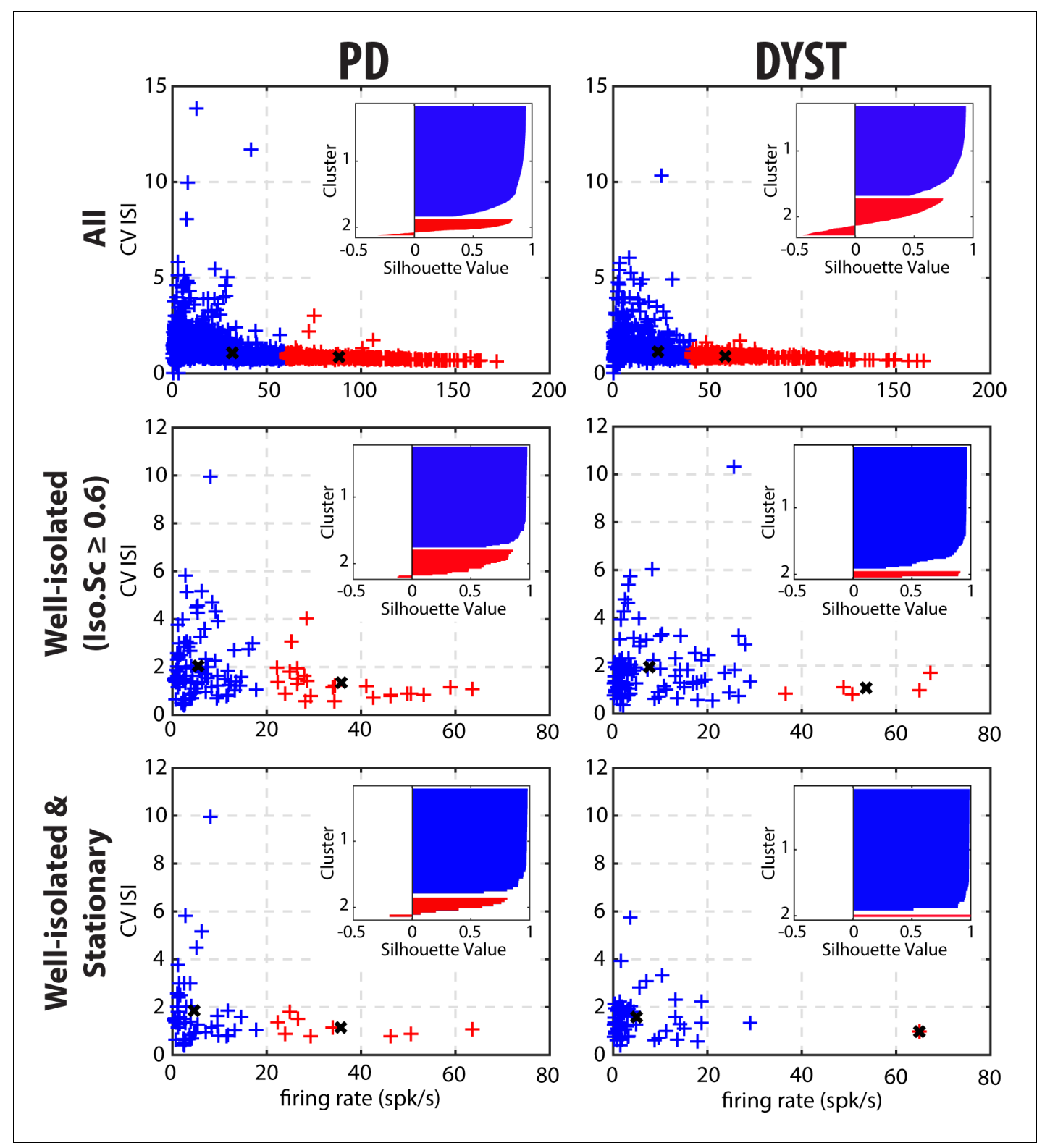

Figure 9. Cluster analysis using discharge properties does not reveal well-separated subpopulations of striatal units in parkinsonian (PD) and dystonic (DYST) patients. 2D k-means cluster analysis with $\mathrm{k}=2$, using the firing rate and the CV of the ISls of each unit as input parameters. Analysis was performed when considering all (upper panels), only the well-isolated (middle panels) and only the well-isolated stationary (lower panels) units. Markers $(x)$ represent cluster centroids. Inset: silhouette values were calculated for each clustering to assess how well-separated the two resulting clusters were. Silhouette values ranges from +1 (data points are very distant from other clusters), through 0 (data points are not distinctly in one cluster or another), to -1 (data points are assigned to the wrong cluster).

The online version of this article includes the following source data for figure 9:

Source data 1. 2D k-means cluster analysis for all units in PD.

Source data 2. 2D k-means cluster analysis for the well-isolated units in PD.

Source data 3. 2D k-means cluster analysis for the well-isolated and stationary units in PD.

Source data 4. 2D k-means cluster analysis for all units in Dystonia.

Source data 5. 2D k-means cluster analysis for the well-isolated units in Dystonia.

Source data 6. 2D k-means cluster analysis for the well-isolated and stationary units in Dystonia. 


\section{Discussion}

There is no consensus as to the impact of aberrant striatal signaling on the spontaneous discharge rate and pattern of the SPNs in PD and Dystonia (Deffains et al., 2016; Ketzef et al., 2017; Liang et al., 2008; Mallet et al., 2006; Sharott et alı, 2017; Singh et al., 2016; Singh et al., 2015). Recordings of the activity of the SPNs are inherently difficult to perform given their very low firing rate and small size, leading to frequent loss and damage of these units (DeLong, 1971). Thus, a particular feature of striatal in vivo extracellular recordings is frequent 'injury activity'. These difficulties can distort the reported population averages of SPNs. For these reasons, we used an automated data-driven approach. A total of 933 and 718 MER segments from the posterior putamen [i.e., sensorimotor area of the striatum (Parent and Hazrati, 1995; Yin and Knowlton, 2006)] of PD and dystonic patients were analyzed. Here, striatal activity was not recorded in the caudate nucleus, but previous studies have shown that putaminal neurons fire at higher rate than the neurons of the caudate nucleus in PD patients (Singh et al., 2016). The striato-pallidal border was automatically detected using a machine learning algorithm (Valsky et al., 2020). MER segments were regularly spaced within the striatum and no manual search for units with low or high discharge rates was performed. Spike detection was performed offline with fully automatic quantification of the isolation quality and stationarity of the identified units. In contrast to previous reports (Singh et al., 2016), we found no drastic modulation in the SPN discharge rate and pattern (compared to values reported for normal/control animals or ET patients) in PD and dystonic patients.

\section{Low discharge rate and similar levels of striatal activity in PD and Dystonia}

We found that the firing rate of the SPNs of both the PD and dystonic patients was extremely sensitive to the quality of the isolation of the units. Specifically, we found a negative correlation between striatal firing rate and isolation score (Figure 2A and B). The negative correlation between the discharge rate of the detected units and their isolation quality revealed that - unlike in the STN (Deffains et al., 2014) - the spike detection and sorting algorithm used in this study and/or the physical properties of the striatal neurons tended to erroneously classify noise events as spikes (false positives) rather than missing real spikes (false negatives). The amplitude of the extracellular action potentials (or the signal-to-noise ratio) is mainly sensitive to the impedance of the microelectrode and the distance between the recorded neurons and the microelectrode, and is not related to firing rate of the neurons. Therefore, we can rule out the possibility that fast firing striatal neurons were less frequently well-isolated and under-represented in the high-score bins. Removing the non-stationary units also reduced the striatal firing rate of SPNs in both the PD and dystonic patients (Figure 3 and Figure 3-figure supplement 1, first column first column). This may indicate that the inclusion of non-stationary ('injured') units can also erroneously increase the striatal firing rate in PD and dystonic patients. Our automatic approach to spike detection, sorting and quality assessment revealed that the median firing rate of the striatal well-isolated stationary units was 3.85 and $2.55 \mathrm{~Hz}$ in PD and dystonic patients, respectively (Figure 3 and Figure 3-figure supplement 1, first column).

Thus, our results are inconsistent with a recent study by Singh et al., 2016 where the SPN discharge rate in PD $(30.2 \pm 1.2 \mathrm{~Hz})$ and dystonic $(9.3 \pm 0.6 \mathrm{~Hz})$ patients increased by $\sim 15$ and 5-fold compared to the low striatal discharge rate $(2.1 \pm 0.1 \mathrm{~Hz})$ found in patients suffering from ET (i.e., a disorder without any known BG malfunction). In the current study, we did not have a control condition, nor did we record the striatal activity from ET patients. Therefore, we cannot rule out the possibility of an increase in the striatal firing rate of both PD and dystonic patients compared to human controls. Moreover, the small fraction of striatal interneurons (including the TANs, presumably the cholinergic interneurons) that was probably included in our sample may have slightly distorted our results (Figure 3 and Figure 3-figure supplement 1). Nevertheless, the putative TANs were hardly distinguishable from the putative SPNs in our human databases (Figure 3-figure supplement 2 and Figure 3-figure supplement 3) and most of the striatal neurons are SPNs (Graveland et al., 1985; Petryszyn et al., 2018; Petryszyn et al., 2014). Finally, in the normal NHP, the discharge rate of the TANs is higher than that of the SPNs, so that their possible inclusion would shift our results towards a higher striatal discharge rate. In any case, we did not find any significant difference between PD and dystonic patients that might reflect a distinct level of striatal hyperactivity in PD 
(Figure 3 and Figure 3-figure supplement 1, first column). The different results between our study and Singh et al., 2016 may be due to: (i) a difference between machine learning detection vs. manual electrophysiologist detection of the striato-pallidal border, (ii) different spike sorting and detection methods, (iii) the use of algorithms for the quantification of the isolation quality and stationarity of the units vs. manual electrophysiologist decision-making, and (iv) different recording techniques (microelectrode type, step size and regular spaced recordings vs. manual search of the recording sites).

\section{Lack of bursty and oscillatory pattern in the SPN spiking activity of PD and dystonic patients}

The emergence of bursty and periodic oscillatory patterns after striatal dopamine depletion has been observed in other BG nuclei than the striatum (Deffains et al., 2018; Deffains et al., 2016; Filion and Tremblay, 1991; Heimer et al., 2002; Raz et al., 2000). Previous studies by our research group reported the emergence of oscillatory spiking activity in the TANs recorded in the striatum of MPTP-treated monkeys, thus indicating that abnormal oscillatory activity following striatal dopamine depletion did not spare the striatum (Deffains et al., 2016; Raz et al., 1996). However, we found no significant parkinsonism-related oscillations in SPN spiking activity (Deffains et al., 2018; Deffains et al., 2016). Here, in line with this finding, we did not observe the emergence of bursting (Figure 3, Figure 3-figure supplement 1, second column and Figure 6) or oscillatory (Figure 3, Figure 3-figure supplement 1, third column and Figure 7) spiking activity of the striatal units sorted in the PD and dystonic patients.

In contrast to our results, Singh and collaborators reported large increases in the bursting activity of the SPNs in PD and dystonic patients (Singh et al., 2016) and MPTP-treated monkeys (Singh et al., 2015). Levodopa-induced increases or decreases of the SPN firing rate may be the result of D1 or D2 receptor activation, respectively (Hernandez-Lopez et al., 2000; Kitai and Surmeier, 1993; West and Grace, 2002). Using the recognized responses to dopaminergic stimulation, Singh et al., 2015 showed that most bursty SPNs in the parkinsonian NHP exhibited a D1 receptor response to levodopa, thus suggesting that the emergence of an abnormal firing pattern predominantly affected the D1 SPNs (i.e., the SPNs of the direct pathway). However, when using a single-cell juxtacellular recording-labeling technique in the 6OHDA rodent, Sharott et al., 2017 showed that in addition to their excessive firing rate, D2 SPNs (that primarily compose the indirect pathway) displayed aberrant phase-locked burst firing to cortical $\beta$ oscillations. In that study, it might be assumed that D2 SPNs, rather than D1 SPNs, would exhibit an abnormal firing rate and pattern in the dopamine-depleted striatum. Therefore, technical advances and further studies in PD patients and animal models should be carried out to reach a consensus.

As reported previously (Deffains et al., 2018; Deffains et al., 2016; Kondabolu et al., 2016; Lemaire et al., 2012; Piña-Fuentes et al., 2018; Silberstein et al., 2003; Singh and Papa, 2019), abnormal oscillations of the LFP have been recorded in the striatum of both PD and dystonic patients (Figure 8A). The LFP most likely represents the sub-threshold (e.g., synaptic input) activity, whereas the MUA reflects the efferent (output) activity of the local neuronal population (Buzsáki et al., 2012; Logothetis, 2003). In both diseases, striatal SPNs were therefore bombarded with pathological oscillations by their major afferent neurons, thus suggesting that the emergence of striatal $\beta$ LFP oscillations is network-driven. Interestingly, other studies have shown that changes in the intrinsic properties of striatal neurons (i.e., increasing SPN excitability through amplification of striatal cholinergic tone) is sufficient to induce robust $\beta$ LFP oscillations in the striatum (McCarthy et al., 2011). Due to the limitations of human extracellular recordings, we were not able to investigate the intrinsic properties of the striatal neurons and therefore cannot rule out the possibility that the intrinsic properties of the striatal neurons played a role in the emergence of $\beta$ LFP oscillations in the striatum. In fact, it is likely that network-based and cell-autonomous mechanisms are not mutually exclusive. In any case, we found no significant phase-locking between striatal spiking activity and monopolar recorded LFP $\beta$ oscillations (Figure 3, Figure 3-figure supplement 1, fourth column and Figure $8 B$ ), thus suggesting that the striatal oscillating synaptic inputs were probably not strong enough to entrain the spiking activity of the striatal SPNs. Indeed, the striatal SPNs failed to express pathological $\beta$ oscillatory activity in either PD or Dystonia. Moreover, recent studies have demonstrated that monopolar and bipolar BG LFPs may be contaminated by the volume conductance of cortical electroencephalogram (EEG) activity (Lalla et al., 2017; Marmor et al., 2017). 
Therefore, it is likely that BG LFPs, including the LFPs recorded in the striatum, do not accurately reflect local cellular activity and should be at best interpreted with caution. Exaggerated striatal LFP oscillations in PD and Dystonia cannot therefore be regarded as direct evidence for the presence of SPN spiking oscillatory activity.

\section{Is the imbalance in the activity of SPN subpopulations evident in PD and Dystonia?}

Obviously, striatal activity must be affected in PD and dystonic patients. A popular theory is that the hypoactivity of the direct pathway (originating from D1 SPNs) and the hyperactivity of the indirect pathway (originating from D2 SPNs) lead to excessive GPi/SNr inhibitory inputs to the thalamus in PD (Wichmann and DeLong, 2003) and vice-versa in Dystonia (Guehl et al., 2009). These aberrant $\mathrm{GPi} / \mathrm{SNr}$ inhibitory inputs to the thalamus lead to the release of abnormal output commands and result in the emergence of the clinical symptoms. Unlike in rodent studies [see e.g., (Sharott et al., 2017)], extracellular recordings of spontaneous activity cannot discriminate between the spiking activity of the striatal D1 and D2 SPNs. However, if striatal dopamine depletion drastically enhances the differences (in discharge rate and pattern) between the two distinct SPN subpopulations (but see Ketzef et al., 2017), one would expect to see a bimodal distribution of SPN discharge properties after dopamine depletion and/or distinct clusters of SPN activity in our patients. Instead, visual inspection of the distributions of the discharge rate (Figure 5) and the CVs of ISls (Figure 6A) revealed long-tailed unimodal distributions in both diseases. Moreover, the cluster analysis using SPN discharge properties failed to identify two well-separated SPN subpopulations (Figure 9).

In conclusion, our results in patients extend our previous study in the MPTP NHP model of PD (Deffains et al., 2016) and studies in the 6-OHDA rodent model (e.g., Ketzef et al., 2017; Maltese et alo, 2019) and demonstrate that abnormal activity along both the direct and indirect pathways of the BG network is not caused by drastic changes in spontaneous SPN spiking activity. Recently, Maltese et al., 2019 reported abnormal recruitment (e.g., during behavior) of D1 and D2 SPNs (which are mostly silent at rest in the healthy condition) in parkinsonism that resulted in an aberrant net balance of striatal outputs, although there were no changes in the firing rate of the individual neurons. Our experimental approach (i.e., extracellular recordings in awake patients in the operating room) does not allow us to estimate the sample size of activated neurons. Moreover, we did not have a control condition and recordings were not performed during behavior to minimize surgical procedure time. Therefore, further experiments in primates (at least in monkeys, if not in patients) are needed to validate or refute this possibility. It is likely that these small to moderate changes in spontaneous SPN discharge are amplified by BG downstream structures (Crompe et al., 2020), thus leading to the clinical symptoms of PD and possibly of Dystonia.

\section{Materials and methods}

\section{Patients and surgery}

Patients with PD and Dystonia were recruited from the movement disorders clinics at the Hadassah Medical Center in Jerusalem. All patients were scheduled to undergo implantation of DBS electrodes into the GPi and underwent MR imaging, and evaluation for motor and non-motor impairments within 30 days prior to surgery. Data were collected from 16 PD and 13 dystonic (non-genetic and genetic Dystonia) patients. Patient demographic information appears in Table 1. Note that our DBS recordings in ET patients started $10 \mathrm{~mm}$ above the thalamic target and therefore did not include striatal recordings. All patients met the criteria for DBS and signed a written informed consent for surgery that involved microelectrode recordings. This study was authorized and approved by the Institutional Review Board of Hadassah Hospital in accordance with the Helsinki Declaration (reference code: 0168-10-HMO).

Surgery was performed using a CRW stereotactic frame (Radionics, Burlington, MA, USA). BG target coordinates were chosen as a composite of the indirect anterior commissure-posterior commissure (AC-PC) atlas-based location and direct (1.5 or 3Tesla) T2 magnetic resonance imaging, using Framelink 4 or 5 software (Medtronic, Minneapolis, USA). All recordings used in this study were made while the patients were fully awake (no sedation or anesthesia) and the PD patients were off dopaminergic medication (overnight washout $>12 \mathrm{hr}$ ). 
Table 1. Patient demographic information.

\begin{tabular}{|c|c|c|c|c|c|c|c|}
\hline Patient no. & Disease & Surgery side & Trajectories & Gender & Age at onset & Age at surgery & Disease duration $(y)$ \\
\hline 1 & PD & bilateral & $\mathrm{R}(2) ; \mathrm{L}(1)$ & M & 40 & 62 & 22 \\
\hline 2 & $P D$ & unilateral & $R(1)$ & $\mathrm{F}$ & 45 & 62 & 17 \\
\hline 3 & PD & bilateral & $\mathrm{R}(2) ; \mathrm{L}(2)$ & $\mathrm{F}$ & 50 & 62 & 12 \\
\hline 4 & PD & unilateral & $L(1)$ & $\mathrm{F}$ & 45 & 62 & 17 \\
\hline 5 & PD & unilateral & $R(2)$ & $\mathrm{F}$ & 49 & 60 & 11 \\
\hline 6 & PD & bilateral & $\mathrm{R}(2) ; \mathrm{L}(2)$ & $\mathrm{F}$ & 66 & 72 & 6 \\
\hline 7 & PD & bilateral & $\mathrm{R}(2) ; \mathrm{L}(1)$ & $\mathrm{F}$ & 43 & 59 & 16 \\
\hline 8 & PD & bilateral & $\mathrm{R}(2) ; \mathrm{L}(2)$ & $\mathrm{F}$ & 54 & 68 & 14 \\
\hline 9 & PD & bilateral & $\mathrm{R}(2) ; \mathrm{L}(2)$ & $\mathrm{F}$ & 55 & 62 & 7 \\
\hline 10 & PD & bilateral & $\mathrm{R}(2) ; \mathrm{L}(2)$ & $\mathrm{F}$ & 46 & 58 & 12 \\
\hline 11 & $P D$ & bilateral & $\mathrm{R}(2) ; \mathrm{L}(2)$ & $\mathrm{F}$ & 41 & 57 & 16 \\
\hline 12 & PD & bilateral & $\mathrm{R}(2) ; \mathrm{L}(2)$ & M & 53 & 66 & 13 \\
\hline 13 & PD & unilateral & $L(2)$ & $\mathrm{F}$ & 53 & 63 & 10 \\
\hline 14 & PD & bilateral & $\mathrm{R}(2) ; \mathrm{L}(2)$ & $\mathrm{M}$ & 35 & 44 & 9 \\
\hline 15 & PD & bilateral & $\mathrm{R}(1) ; \mathrm{L}(1)$ & M & 41 & 57 & 16 \\
\hline 16 & PD & unilateral & $R(2)$ & M & 50 & 57 & 7 \\
\hline 17 & Dystonia (NG) & bilateral & $\mathrm{R}(1) ; \mathrm{L}(2)$ & $\mathrm{F}$ & 36 & 56 & 20 \\
\hline 18 & Dystonia (NG) & bilateral & $\mathrm{R}(2) ; \mathrm{L}(2)$ & M & 44 & 49 & 5 \\
\hline 19 & Dystonia (NG) & bilateral & $\mathrm{R}(1) ; \mathrm{L}(2)$ & $\mathrm{F}$ & 45 & 65 & 20 \\
\hline 20 & Dystonia (NG) & bilateral & $\mathrm{R}(2) ; \mathrm{L}(1)$ & $\mathrm{F}$ & 58 & 60 & 2 \\
\hline 21 & Dystonia (NG) & bilateral & $\mathrm{R}(2) ; \mathrm{L}(2)$ & $\mathrm{F}$ & 13 & 19 & 6 \\
\hline 22 & Dystonia (NG) & bilateral & $R(1) ; L(1)$ & $\mathrm{F}$ & 60 & 63 & 2 \\
\hline 23 & Dystonia (NG) & bilateral & $\mathrm{R}(2) ; \mathrm{L}(2)$ & M & 60 & 62 & 2 \\
\hline 24 & Dystonia(NG) & bilateral & $\mathrm{R}(2) ; \mathrm{L}(2)$ & $\mathrm{F}$ & 69 & 71 & 2 \\
\hline 25 & Dystonia (G) & bilateral & $\mathrm{R}(2) ; \mathrm{L}(2)$ & M & 16 & 25 & 9 \\
\hline 26 & Dystonia (G) & bilateral & $\mathrm{R}(2) ; \mathrm{L}(2)$ & M & 12 & 18 & 6 \\
\hline 27 & Dystonia (G) & bilateral & $\mathrm{R}(1) ; \mathrm{L}(2)$ & M & 24 & 39 & 15 \\
\hline 28 & Dystonia (G) & bilateral & $\mathrm{R}(2) ; \mathrm{L}(2)$ & $\mathrm{M}$ & 39 & 54 & 15 \\
\hline 29 & Dystonia (G) & bilateral & $R(1) ; L(2)$ & M & 53 & 56 & 3 \\
\hline
\end{tabular}

PD patients (5 males and 11 females) were $60.7 \pm 6.1$ years old and with a disease duration of $12.8 \pm 4.4$ years (mean \pm standard deviation, SD). Dystonic patients (7 males and six females) were $49.0 \pm 18.0$ years old and with a disease duration of $8.2 \pm 6.9$ years (mean \pm standard deviation, SD). NG: nongenetic; G: genetic; R: right; L: left; Numbers in parentheses indicate the number of microelectrode trajectories; M: male; F: female.

\section{Data acquisition}

The data were acquired using two systems: MicroGuide (prior to 2015, previously described [Deffains et al., 2014]) and Neuro Omega (from 2015, previously described [Valsky et al., 2020]).

\section{Microelectrode recordings}

For every recording session, a microelectrode recording (MER) exploration using one or two microelectrode trajectories ( $2 \mathrm{~mm}$ apart) was made starting $15 \mathrm{~mm}$ above the pre-operative T2 MRI image-based calculated target. Our trajectories followed a double-oblique approach (50 to $80^{\circ}$ from the axial AC-PC plane and 0 to $10^{\circ}$ degrees from the midsagittal plane) through the posterior putamen and the GPe and towards the ventral border of the posterior-lateral portion of the GPi target (Figure 1 and Video 1). The target coordinates were in the range of the approximate anatomic coordinates for the motor domain of the GPi: Lateral $(X)=19-22 \mathrm{~mm}$ from the midline or $\sim 18 \mathrm{~mm}$ 
from the third ventricle wall; Anterior $(Y)=1-3.5 \mathrm{~mm}$ from the mid-commissural point (MCP), and vertical $(Z)=-1$ to -4 below the AC-PC plane.

The 'central' electrode was directed at the ventral border of the posterior-lateral portion of the GPi and the 'anterior' electrode was located $2 \mathrm{~mm}$ anterior/ventral to the central electrode in the parasagittal plane. Some of these recordings were made by a single microelectrode trajectory (instead of two) to accommodate cortical anatomy under the burr hole and brain blood vessels (Machado et al., 2006).

MER segments were regularly sampled in space in order to avoid recording bias towards particular striatal units. For all our GPi-DBS surgeries, the step size between two MER segments ranged from 100 to $200 \mu \mathrm{m}$ and was controlled by the neurophysiologist to achieve optimal identification of the pallidal borders. At each step, MER segments were recorded from 4 to $140 \mathrm{~s}$ (after a $2 \mathrm{~s}$ signal stabilization period).

A total of 93 microelectrode trajectories aiming at the GPi were analyzed (48 in PD and 45 in Dystonia), yielding a total of 933 and 718 MER segments within the posterior putamen of patients suffering from PD and Dystonia, respectively. Striatum-GPe borders were automatically detected using a machine learning software (Valsky et alo, 2020).

\section{Group-based microelectrode track trajectories}

The trajectories of GPi DBS patients were reconstructed using the open source LeadDBS program (www.lead-dbs.org) using LeadDBS v2.2.3 (Horn et al., 2019; Horn and Kühn, 2015). Lead Group, implemented within the Lead-DBS environment (Treu et al., 2020), was used to graphically illustrate the group-based microelectrode track trajectories within the posterior putamen. Pre-operative imaging was performed on a $1.5 \mathrm{~T}$ or $3 \mathrm{~T} \mathrm{MRI}$ and included a pre-operative T1 and T2 sequence. All patients received post-operative CT. The post-operative CT was co-registered to anchor modality with the pre-operative T1 MRI, using a two-stage linear registration (rigid followed by affine) as implemented in Advanced Normalization Tools (Avants et al., 2008). Linear co-registration between the MR T1 and T2 modalities was done using SPM12 (Penny et al., 2007). Pre- (and post-) operative acquisitions were spatially normalized into MNI_ICBM_2009b_NLIN_ASYM space (Fonov et al., 2011) based on pre-operative acquisitions using the Symmetric Normalization (SyN) registration approach as implemented in Advanced Normalization Tools (Avants et alo, 2008). Nonlinear deformation into template space was achieved in five stages. After two linear (rigid followed by affine) steps, a nonlinear (whole brain) SyN-registration stage was followed by two nonlinear SyN-registrations that consecutively focused on the GP as defined by subcortical masks in Schönecker et al., 2009. DBS electrode localizations were corrected for brain shift in post-operative acquisitions by applying a refined affine transform calculated between the pre- and post-operative acquisitions that were restricted to the GP as implemented in the brain shift-correction module of Lead-DBS software (Horn and Kühn, 2015). DBS electrodes were automatically pre-localized in native and template space using the PaCER algorithm (Husch et alo, 2018). DBS electrodes were manually localized based on post-operative acquisitions using a tool specifically designed for this task.

A total of 36 microelectrode track trajectories (13 in PD and 23 in Dystonia) of four PD and six dystonic patients were visualized (Figure 1). We failed to include some patients because of corrupted or missing pre- or post-operative images. A $100 \mu \mathrm{m}$ T1 scan of an ex-vivo human brain, acquired on a 7T MRI scanner (Edllow et al., 2019) served as a background template in Figure 1. The definition of the striatum, GPe and GPi boundaries was informed by the Distal atlas (Ewert et al., 2018). For further visualization of microelectrode track trajectories in the posterior putamen see Video 1.

\section{Offline spike sorting and assessment of isolation quality and stationarity of sorted units}

To avoid human biases that could corrupt spike detection and sorting outcomes (Pedreira et alo, 2012; Rey et al., 2015), single-unit striatal activity was assessed by sorting the spike trains from each MER segment recorded within the striatum using an automatic offline spike detection and sorting method (Offline Sorter v4.4.2.0, Plexon Inc, Dallas, Texas, RRID:SCR_000012). For each bandpass filtered spiking signal (multi-unit activity, MUA), spikes were detected using a negative voltage threshold trigger systematically set at 3SDs from the mean of its peak height histogram. Salient 
features of the detected spikes were extracted with principal component analysis, using the scores of the first two principal components as features for clustering. Then, identification of different spike clusters reflecting the activity of different units was done by an automatic k-means clustering algorithm (K-Means Scan with a number of clusters between 1 and 5 and a circular seed cluster pattern for choosing the initial cluster centers). The clustering that produced the best value of the Pseudo- $F$ statistic was then chosen as the clustering pattern for that band-pass filtered spiking signal. Finally, the isolation quality of the units identified by the spike sorter software was systematically graded by measuring their isolation scores using an independent algorithm (Joshua et al., 2007). The isolation score ranges from 0 (i.e. highly noisy) to 1 (i.e. perfect isolation).

To assess the stationarity of the firing rate and spike amplitude of each well-isolated unit (isolation score $\geq 0.6$ ), we segmented the data comprised between the first and the last spike of its spike train into 10 equal non-overlapping time bins. Then, we Z-score normalized the firing rate and the average spike amplitude in each bin using the mean and the SD of either the firing rate or the average spike amplitude calculated over the 10 bins. Finally, the slope value of the linear regression line for the firing rate and the average spike amplitude were calculated and expressed in z-score/bin. Units with slope values of the linear regression line for the firing rate or average spike amplitude that were greater than or equal to the 70th percentile were defined as non-stationary units.

\section{Discharge pattern assessment of spike train}

For each spike train of the sorted units, we calculated the inter-spike intervals (ISI) and generated their ISI histograms. In parallel, we also computed the autocorrelograms of the spike train of the well-isolated stationary units, calculated for $\pm 500 \mathrm{~ms}$ offset with $10 \mathrm{~ms}$-bins. For each autocorrelogram, values were normalized so that autocorrelation values ranged from 0 to 1 .

For burst detection, we applied the Poisson surprise method with the surprise maximization (SM) search algorithm (Legéndy and Salcman, 1985) to each spike train of the well-isolated stationary units, using the following parameters: minimal burst length $=3$ spikes; threshold surprise value $(S)=10$; burst ISI limit $=$ mean $(I S I) / 2$; add limit $=150 \%$ of burst ISI limit and inclusion criteria $(\mathrm{IC})=5$.

For each spike train of the well-isolated stationary units, the frequency (number of bursts/s) and mean duration (s) of the burst episodes were calculated over their entire recording span. These two metrics were used to determine the burst prevalence for each unit which was defined as the burst frequency * mean burst duration. For each unit, the burst prevalence (range: 0-1) represents the probability that the discharge pattern is bursty.

\section{Power spectral density}

Prior to power spectral density (PSD) calculations, each band-pass filtered spiking signal (multi-unit activity, MUA) recorded in the vicinity of a sorted unit was Z-score normalized (using its mean and SD) to obtain an unbiased estimate (by the electrode impedance, the A/D characteristics of the recording system, or the amplitude of the recorded neuronal activity) of the oscillatory activity (Zaidel et al., 2010). The Z-normalized signal was rectified by the 'absolute' operator (Deffains et alı, 2018; Deffains et alı, 2016; Deffains et al., 2014; Moran et al., 2008; Zaidel et al., 2010). The rectified signal follows the envelope of the MUA and therefore enables the detection of periodic oscillatory activities with frequencies below the range of the online band-pass filter. Since the LFP frequency domain was filtered out, the resulting PSD only represented the oscillatory features of the spiking activity. Although up to five units could be sorted from each MUA (see above in Offline spike sorting and assessment of isolation quality and stationarity of sorted units), each MUA was only analyzed once.

The PSD of each rectified Z-normalized signal (expressed in z-score $2 / \mathrm{Hz}$ ) was calculated using Welch's method with a $1 \mathrm{~s}$ Hamming window (50\% overlap) and a spectral resolution of $1 \mathrm{~Hz}$ ( $\mathrm{nfft}=44000$ or 48000 , sampling frequency $=44$ or $48 \mathrm{kHz}$ depending on the acquisition system). To evaluate the $\beta$ power, the baseline values in the 13-30 Hz range of each PSD were linearly interpolated [based on the two closest points that flanked the $13-30 \mathrm{~Hz}$ band, namely the values at 12 and $31 \mathrm{~Hz}$ (spectral resolution of $1 \mathrm{~Hz}$, see above)] and averaged. Then, the $\beta$ power (i.e., the mean of the observed values between 13 and $30 \mathrm{~Hz}$ ) was compared to the interpolated $\beta$ power (baseline). 
Similarly, we also calculated the PSD of the striatal monopolar $(0.1-300 \mathrm{~Hz})$ LFP. To do so, the PSD of each Z-normalized LFP was calculated using Welch's method recorded in the vicinity of the cells (see above for the parameters; but $\mathrm{nfft}=1375$, sampling frequency $=1.375 \mathrm{kHz}$ ) and without prior rectification by the absolute operator.

\section{Spike-triggered average of the LFP}

To investigate the spike-LFP relationship in the temporal domain, we also calculated the spike-triggered average (STA) of the LFPs (Deffains et alı, 2016; Goldberg et al., 2004). To do so, the LFP was recorded in the vicinity of the sorted units (i.e., spiking activity and LFP were recorded on the same electrode). Each Z-normalized LFP was offline band-pass filtered from 4 to $12 \mathrm{~Hz}$ or from 13 to $30 \mathrm{~Hz}$ (4-pole Butterworth filter, filtfilt Matlab function). For comparison, STAs-LFP were also calculated after randomly shifting the timestamp of each spike of the spike train [i.e., random time (comprised between 0 and $1 \mathrm{~s}$ ) was added to the timestamp of each spike of the spike train] in order to abolish any relationship between LFP and spiking activity (Shift predictor). The PSD of the STA-LFP was calculated as for the LFP.

\section{Software and statistics}

Anatomical analysis was done with the open source LeadDBS program (www.lead-dbs.org). All the physiological data and statistical analyses were carried out using MATLAB R2016a routines (Mathworks, Natick, MA, USA, RRID:SCR_001622). Mann-Whitney U-tests and Wilcoxon signed rank tests were used for statistical comparisons of two unpaired and paired sample means, respectively. Statistical comparisons of two sample proportions were performed using Chi-square tests. Analysis of the subset of $\geq 10$ s-MER segments with striatal well-isolated stationary units yielded similar results (Figure 3-figure supplement 5 and Figure 6-figure supplement 1) to those reported here, thus indicating that our results were not confounded by the shortest MER segments. The criterion for statistical significance was set at $p<0.05$ for all statistical tests.

\section{Acknowledgements}

We thank Andreas Horn and Simon Oxenford for their assistance in illustrating the group-based microelectrode track trajectories using Lead-DBS software, Atira Bick for pre- and post-operative imaging, Esther Singer for editing the manuscript and the patients for agreement to participate in the study and authorizing the use of their electrophysiological recordings. This study was supported by the European Research Council (ERC), Rosetrees, Israel Science Foundation (ISF) and Israel Authority for Innovation grants to $\mathrm{HB}$, and the French National Research Agency (ANR) and the French National Center for Scientific Research (CNRS) to MD.

\section{Additional information}

Funding

\begin{tabular}{ll} 
Funder & Author \\
\hline European Research Council & Hagai Bergman \\
\hline Rosetrees & Hagai Bergman \\
\hline Israel Science Foundation & Hagai Bergman \\
\hline Israel Authority for Innovation & Hagai Bergman \\
\hline $\begin{array}{l}\text { French National Research } \\
\text { Agency }\end{array}$ & Marc Deffains \\
\hline $\begin{array}{l}\text { French National Center for } \\
\text { Scientific Research }\end{array}$ & Marc Deffains \\
\hline
\end{tabular}

The funders had no role in study design, data collection and interpretation, or the decision to submit the work for publication. 
Author contributions

Dan Valsky, Conceptualization, Software, Formal analysis, Investigation, Methodology, Writing - original draft, Writing - review and editing; Shai Heiman Grosberg, Software, Formal analysis, Writing review and editing; Zvi Israel, Conceptualization, Resources, Supervision, Project administration, Writing - review and editing; Thomas Boraud, Conceptualization, Writing - review and editing; Hagai Bergman, Conceptualization, Resources, Supervision, Funding acquisition, Investigation, Methodology, Writing - original draft, Project administration, Writing - review and editing; Marc Deffains, Conceptualization, Software, Formal analysis, Supervision, Investigation, Methodology, Writing - original draft, Project administration, Writing - review and editing

\section{Author ORCIDs}

Dan Valsky (iD https://orcid.org/0000-0002-2385-424X

Hagai Bergman (iD http://orcid.org/0000-0002-2402-6673

Marc Deffains (D) https://orcid.org/0000-0003-0734-6541

Ethics

Human subjects: All patients met the criteria for DBS and signed a written informed consent for surgery that involved microelectrode recording. This study was authorized and approved by the Institutional Review Board of Hadassah Hospital in accordance with the Helsinki Declaration (reference code: 0168-10-HMO).

Decision letter and Author response

Decision letter https://doi.org/10.7554/eLife.57445.sa1

Author response https://doi.org/10.7554/eLife.57445.sa2

\section{Additional files}

Supplementary files

- Transparent reporting form

Data availability

All data generated or analysed during this study are included in the manuscript and supporting files. Source data files have been provided for Figures 2, 3 and 9.

\section{References}

Albin RL, Young AB, Penney JB. 1989. The functional anatomy of basal ganglia disorders. Trends in Neurosciences 12:366-375. DOI: https://doi.org/10.1016/0166-2236(89)90074-X, PMID: 2479133

Aosaki T, Miura M, Suzuki T, Nishimura K, Masuda M. 2010. Acetylcholine-dopamine balance hypothesis in the striatum: an update. Geriatrics \& Gerontology International 10:S148-S157. DOI: https://doi.org/10.1111/j. 1447-0594.2010.00588.x

Avants BB, Epstein CL, Grossman M, Gee JC. 2008. Symmetric diffeomorphic image registration with crosscorrelation: evaluating automated labeling of elderly and neurodegenerative brain. Medical Image Analysis 12 : 26-41. DOI: https://doi.org/10.1016/j.media.2007.06.004, PMID: 17659998

Benarroch EE. 2012. Effects of acetylcholine in the striatum recent insights and therapeutic implications. Neurology 79:274-281. DOI: https://doi.org/10.1212/WNL.0b013e31825fe154, PMID: 22802594

Bergman H, Wichmann T, DeLong MR. 1990. Reversal of experimental parkinsonism by lesions of the subthalamic nucleus. Science 249:1436-1438. DOl: https://doi.org/10.1126/science.2402638, PMID: 2402638

Bolam JP, Ellender TJ. 2016. Histamine and the striatum. Neuropharmacology 106:74-84. DOI: https://doi.org/ 10.1016/j.neuropharm.2015.08.013, PMID: 26275849

Bonsi P, Cuomo D, Martella G, Madeo G, Schirinzi T, Puglisi F, Ponterio G, Pisani A. 2011. Centrality of striatal cholinergic transmission in basal ganglia function. Frontiers in Neuroanatomy 5:6. DOI: https://doi.org/10.3389/ fnana.2011.00006, PMID: 21344017

Bostan AC, Dum RP, Strick PL. 2013. Cerebellar networks with the cerebral cortex and basal ganglia. Trends in Cognitive Sciences 17:241-254. DOI: https://doi.org/10.1016/j.tics.2013.03.003, PMID: 23579055 
Brown P. 2003. Oscillatory nature of human basal ganglia activity: relationship to the pathophysiology of Parkinson's disease. Movement Disorders 18:357-363. DOI: https://doi.org/10.1002/mds.10358, PMID: 12671 940

Brunel N, Hakim V. 2008. Sparsely synchronized neuronal oscillations. Chaos: An Interdisciplinary Journal of Nonlinear Science 18:015113. DOI: https://doi.org/10.1063/1.2779858

Buzsáki G, Anastassiou CA, Koch C. 2012. The origin of extracellular fields and currents-EEG, ECoG, LFP and spikes. Nature Reviews Neuroscience 13:407-420. DOI: https://doi.org/10.1038/nrn3241, PMID: 22595786

Chen CC, Brücke C, Kempf F, Kupsch A, Lu CS, Lee ST, Tisch S, Limousin P, Hariz M, Brown P. 2006. Deep brain stimulation of the subthalamic nucleus: a two-edged sword. Current Biology 16:R952-R953. DOI: https://doi. org/10.1016/j.cub.2006.10.013, PMID: 17113373

Crompe B, Aristieta A, Leblois A, Elsherbiny S, Boraud T, Mallet NP. 2020. The globus pallidus orchestrates abnormal network dynamics in a model of parkinsonism. Nature Communications 11:1570. DOI: https://doi. org/10.1038/s41467-020-15352-3, PMID: 32218441

Crutcher MD, DeLong MR. 1984. Single cell studies of the primate putamen I functional organization. ExpBrain Res 53:233-243. DOI: https://doi.org/10.1007/BF00238153

Deffains M, Legallet E, Apicella P. 2010. Modulation of neuronal activity in the monkey putamen associated with changes in the habitual order of sequential movements. Journal of Neurophysiology 104:1355-1369. DOI: https://doi.org/10.1152/jn.00355.2010, PMID: 20610785

Deffains M, Holland P, Moshel S, Ramirez de Noriega F, Bergman H, Israel Z. 2014. Higher neuronal discharge rate in the motor area of the subthalamic nucleus of parkinsonian patients. Journal of Neurophysiology 112: 1409-1420. DOI: https://doi.org/10.1152/jn.00170.2014, PMID: 24920022

Deffains M, Iskhakova L, Katabi S, Haber SN, Israel Z, Bergman H. 2016. Subthalamic, not striatal, activity correlates with basal ganglia downstream activity in normal and parkinsonian monkeys. eLife 5:e16443. DOI: https://doi.org/10.7554/eLife.16443, PMID: 27552049

Deffains M, Iskhakova L, Katabi S, Israel Z, Bergman H. 2018. Longer $\beta$ oscillatory episodes reliably identify pathological subthalamic activity in parkinsonism. Movement Disorders 33:1609-1618. DOI: https://doi.org/10. 1002/mds.27418, PMID: 30145811

Deffains M, Bergman H. 2015. Striatal cholinergic interneurons and cortico-striatal synaptic plasticity in health and disease. Movement Disorders 30:1014-1025. DOI: https://doi.org/10.1002/mds.26300, PMID: 26095280

Deffains M, Bergman H. 2019. Parkinsonism-related $\beta$ oscillations in the primate basal ganglia networks - Recent advances and clinical implications. Parkinsonism \& Related Disorders 59:2-8. DOI: https://doi.org/10.1016/j. parkreldis.2018.12.015, PMID: 30578010

DeLong MR. 1971. Activity of pallidal neurons during movement. Journal of Neurophysiology 34:414-427. DOI: https://doi.org/10.1152/jn.1971.34.3.414, PMID: 4997823

Edlow BL, Mareyam A, Horn A, Polimeni JR, Witzel T, Tisdall MD, Augustinack JC, Stockmann JP, Diamond BR, Stevens A, Tirrell LS, Folkerth RD, Wald LL, Fischl B, van der Kouwe A. 2019. 7 tesla MRI of the ex vivo human brain at 100 micron resolution. Scientific Data 6:244. DOI: https://doi.org/10.1038/s41597-019-0254-8, PMID: 31666530

Ewert S, Plettig P, Li N, Chakravarty MM, Collins DL, Herrington TM, Kühn AA, Horn A. 2018. Toward defining deep brain stimulation targets in MNI space: a subcortical atlas based on multimodal MRI, histology and structural connectivity. Neurolmage 170:271-282. DOI: https://doi.org/10.1016/j. neuroimage.2017.05.015, PMID: 28536045

Filion M, Tremblay Le. 1991. Abnormal spontaneous activity of globus pallidus neurons in monkeys with MPTPinduced parkinsonism. Brain Research 547:140-144. DOI: https://doi.org/10.1016/0006-8993(91)90585-J

Fonov V, Evans AC, Botteron K, Almli CR, McKinstry RC, Collins DL, Brain Development Cooperative Group. 2011. Unbiased average age-appropriate atlases for pediatric studies. Neurolmage 54:313-327. DOI: https:// doi.org/10.1016/j.neuroimage.2010.07.033, PMID: 20656036

Fox SH, Chuang R, Brotchie JM. 2009. Serotonin and Parkinson's disease: On movement, mood, and madness. Movement Disorders 24:1255-1266. DOl: https://doi.org/10.1002/mds.22473, PMID: 19412960

Fremont R, Tewari A, Angueyra C, Khodakhah K. 2017. A role for cerebellum in the hereditary dystonia DYT1. eLife 6:e22775. DOI: https://doi.org/10.7554/eLife.22775, PMID: 28198698

Gerfen CR, Engber TM, Mahan LC, Susel Z, Chase TN, Monsma FJ, Sibley DR. 1990. D1 and D2 dopamine receptor-regulated gene expression of striatonigral and striatopallidal neurons. Science 250:1429-1432. DOI: https://doi.org/10.1126/science.2147780, PMID: 2147780

Goldberg JA, Rokni U, Boraud T, Vaadia E, Bergman H. 2004. Spike synchronization in the cortex/basal-ganglia networks of parkinsonian primates reflects global dynamics of the local field potentials. Journal of Neuroscience 24:6003-6010. DOI: https://doi.org/10.1523/JNEUROSCI.4848-03.2004, PMID: 15229247

Graveland GA, Williams RS, Difiglia M. 1985. A Golgi study of the human neostriatum: Neurons and afferent fibers. The Journal of Comparative Neurology 234:317-333. DOI: https://doi.org/10.1002/cne.902340304

Guehl D, Cuny E, Ghorayeb I, Michelet T, Bioulac B, Burbaud P. 2009. Primate models of dystonia. Progress in Neurobiology 87:118-131. DOI: https://doi.org/10.1016/j.pneurobio.2008.10.003

Heimer G, Bar-Gad I, Goldberg JA, Bergman H. 2002. Dopamine Replacement Therapy Reverses Abnormal Synchronization of Pallidal Neurons in the 1-Methyl-4-Phenyl-1,2,3,6-Tetrahydropyridine Primate Model of Parkinsonism. The Journal of Neuroscience 22:7850-7855. DOI: https://doi.org/10.1523/JNEUROSCI.22-1807850.2002

Helmich RC, Hallett M, Deuschl G, Toni I, Bloem BR. 2012. Cerebral causes and consequences of parkinsonian resting tremor: a tale of two circuits? Brain 135:3206-3226. DOI: https://doi.org/10.1093/brain/aws023 
Helmich RC. 2018. The cerebral basis of parkinsonian tremor: a network perspective. Movement Disorders 33 : 219-231. DOI: https://doi.org/10.1002/mds.27224, PMID: 29119634

Hernandez-Lopez S, Tkatch T, Perez-Garci E, Galarraga E, Bargas J, Hamm H, Surmeier DJ. 2000. D2 dopamine receptors in striatal medium spiny neurons reduce L-type Ca2+ currents and excitability via a novel PLC[beta]1IP3-calcineurin-signaling cascade. The Journal of Neuroscience 20:8987-8995. PMID: 11124974

Horn A, Li N, Dembek TA, Kappel A, Boulay C, Ewert S, Tietze A, Husch A, Perera T, Neumann WJ, Reisert M, Si H, Oostenveld R, Rorden C, Yeh FC, Fang Q, Herrington TM, Vorwerk J, Kühn AA. 2019. Lead-DBS v2: towards a comprehensive pipeline for deep brain stimulation imaging. Neurolmage 184:293-316. DOI: https://doi.org/ 10.1016/j.neuroimage.2018.08.068, PMID: 30179717

Horn A, Kühn AA. 2015. Lead-DBS: a toolbox for deep brain stimulation electrode localizations and visualizations. Neurolmage 107:127-135. DOI: https://doi.org/10.1016/j.neuroimage.2014.12.002, PMID: 254 98389

Hoshi E, Tremblay L, Féger J, Carras PL, Strick PL. 2005. The cerebellum communicates with the basal ganglia. Nature Neuroscience 8:1491-1493. DOI: https://doi.org/10.1038/nn1544, PMID: 16205719

Husch A, Petersen MV, Gemmar P, Goncalves J, Hertel F. 2018. PaCER - A fully automated method for electrode trajectory and contact reconstruction in deep brain stimulation. Neurolmage: Clinical 17:80-89. DOI: https:// doi.org/10.1016/j.nicl.2017.10.004, PMID: 29062684

Joshua M, Elias S, Levine O, Bergman H. 2007. Quantifying the isolation quality of extracellularly recorded action potentials. Journal of Neuroscience Methods 163:267-282. DOI: https://doi.org/10.1016/j.jneumeth.2007.03. 012, PMID: 17477972

Kaneoke Y, Vitek JL. 1996. Burst and oscillation as disparate neuronal properties. Journal of Neuroscience Methods 68:211-223. DOI: https://doi.org/10.1016/0165-0270(96)00081-7, PMID: 8912194

Ketzef M, Spigolon G, Johansson Y, Bonito-Oliva A, Fisone G, Silberberg G. 2017. Dopamine depletion impairs bilateral sensory processing in the striatum in a Pathway-Dependent manner. Neuron 94:855-865. DOI: https:// doi.org/10.1016/j.neuron.2017.05.004, PMID: 28521136

Kimura M, Kato M, Shimazaki H. 1990. Physiological properties of projection neurons in the monkey striatum to the globus pallidus. Experimental Brain Research 82:672-676. DOI: https://doi.org/10.1007/BF00228811, PMID: 1705520

Kish LJ, Palmer MR, Gerhardt GA. 1999. Multiple single-unit recordings in the striatum of freely moving animals: effects of apomorphine and D-amphetamine in normal and unilateral 6-hydroxydopamine-lesioned rats. Brain Research 833:58-70. DOI: https://doi.org/10.1016/S0006-8993(99)01496-1, PMID: 10375677

Kitai ST, Surmeier DJ. 1993. Cholinergic and dopaminergic modulation of potassium conductances in neostriatal neurons. Advances in Neurology 60:40-52. PMID: 8380527

Kondabolu K, Roberts EA, Bucklin M, McCarthy MM, Kopell N, Han X. 2016. Striatal cholinergic interneurons generate beta and gamma oscillations in the corticostriatal circuit and produce motor deficits. PNAS 113: E3159-E3168. DOI: https://doi.org/10.1073/pnas.1605658113, PMID: 27185924

Kopell N, LeMasson G. 1994. Rhythmogenesis, amplitude modulation, and multiplexing in a cortical architecture. PNAS 91:10586-10590. DOI: https://doi.org/10.1073/pnas.91.22.10586, PMID: 7937997

Kuhn AA, Trottenberg T, Kivi A, Kupsch A, Schneider GH, Brown P. 2005. The relationship between local field potential and neuronal discharge in the subthalamic nucleus of patients with Parkinson's disease. ExpNeurol 194:212-220. DOI: https://doi.org/10.1016/j.expneurol.2005.02.010

Lalla L, Rueda Orozco PE, Jurado-Parras MT, Brovelli A, Robbe D. 2017. Local or not local: investigating the nature of striatal theta oscillations in behaving rats. Eneuro 4:ENEURO.0128-17.2017. DOI: https://doi.org/10. 1523/ENEURO.0128-17.2017, PMID: 28966971

Legéndy CR, Salcman M. 1985. Bursts and recurrences of bursts in the spike trains of spontaneously active striate cortex neurons. Journal of Neurophysiology 53:926-939. DOI: https://doi.org/10.1152/jn.1985.53.4.926, PMID: 3998798

Lemaire N, Hernandez LF, Hu D, Kubota Y, Howe MW, Graybiel AM. 2012. Effects of dopamine depletion on LFP oscillations in striatum are task- and learning-dependent and selectively reversed by L-DOPA. PNAS 109: 18126-18131. DOI: https://doi.org/10.1073/pnas.1216403109, PMID: 23074253

Liang L, DeLong MR, Papa SM. 2008. Inversion of dopamine responses in striatal medium spiny neurons and involuntary movements. Journal of Neuroscience 28:7537-7547. DOI: https://doi.org/10.1523/JNEUROSCl. 1176-08.2008, PMID: 18650331

Limousin P, Krack P, Pollak P, Benazzouz A, Ardouin C, Hoffmann D, Benabid AL. 1998. Electrical stimulation of the subthalamic nucleus in advanced Parkinson's disease. New England Journal of Medicine 339:1105-1111. DOI: https://doi.org/10.1056/NEJM199810153391603, PMID: 9770557

Logothetis NK. 2003. The underpinnings of the BOLD functional magnetic resonance imaging signal. The Journal of Neuroscience 23:3963-3971. DOI: https://doi.org/10.1523/JNEUROSCI.23-10-03963.2003، PMID: 12764080

Machado A, Rezai AR, Kopell BH, Gross RE, Sharan AD, Benabid AL. 2006. Deep brain stimulation for parkinson's disease: surgical technique and perioperative management. Movement Disorders 21:S247-S258. DOI: https://doi.org/10.1002/mds.20959, PMID: 16810722

Mallet N, Ballion B, Le Moine C, Gonon F. 2006. Cortical inputs and GABA interneurons imbalance projection neurons in the striatum of parkinsonian rats. Journal of Neuroscience 26:3875-3884. DOI: https://doi.org/10. 1523/JNEUROSCI.4439-05.2006, PMID: 16597742

Maltese M, March JR, Bashaw AG, Tritsch NX. 2019. Dopamine modulates the size of striatal projection neuron ensembles. bioRxiv. DOI: https://doi.org/10.1101/865006 
Marmor O, Valsky D, Joshua M, Bick AS, Arkadir D, Tamir I, Bergman H, Israel Z, Eitan R. 2017. Local vs. volume conductance activity of field potentials in the human subthalamic nucleus. Journal of Neurophysiology 117: 2140-2151. DOI: https://doi.org/10.1152/jn.00756.2016, PMID: 28202569

McCarthy MM, Moore-Kochlacs C, Gu X, Boyden ES, Han X, Kopell N. 2011. Striatal origin of the pathologic beta oscillations in Parkinson's disease. PNAS 108:11620-11625. DOI: https://doi.org/10.1073/pnas. 1107748108

Menegas W, Bergan JF, Ogawa SK, Isogai Y, Umadevi Venkataraju K, Osten P, Uchida N, Watabe-Uchida M. 2015. Dopamine neurons projecting to the posterior striatum form an anatomically distinct subclass. eLife 4: e10032. DOI: https://doi.org/10.7554/eLife.10032, PMID: 26322384

Mink JW. 1996. The basal ganglia: focused selection and inhibition of competing motor programs. Progress in Neurobiology 50:381-425. DOI: https://doi.org/10.1016/S0301-0082(96)00042-1, PMID: 9004351

Moran A, Bergman H, Israel Z, Bar-Gad I. 2008. Subthalamic nucleus functional organization revealed by parkinsonian neuronal oscillations and synchrony. Brain 131:3395-3409. DOI: https://doi.org/10.1093/brain/ awn270, PMID: 18986993

Moro E, LeReun C, Krauss JK, Albanese A, Vidailhet M. 2017. Efficacy of pallidal stimulation in isolated dystonia: a systematic review and meta-analysis. European Journal of Neurology 9:552-560. DOI: https://doi.org/10. 1111/ene.13255

Moshel S, Shamir RR, Raz A, de Noriega FR, Eitan R, Bergman H, Israel Z. 2013. Subthalamic nucleus long-range synchronization-an independent hallmark of human Parkinson's disease. Front SystNeurosci 7:79. DOI: https:// doi.org/10.3389/fnsys.2013.00079

Odekerken VJ, Boel JA, Schmand BA, de Haan RJ, Figee M, van den Munckhof P, Schuurman PR, de Bie RM, NSTAPS study group. 2016. GPi vs STN deep brain stimulation for parkinson disease: three-year follow-up. Neurology 86:755-761. DOI: https://doi.org/10.1212/WNL.0000000000002401, PMID: 26819458

Ostrem JL, San Luciano M, Dodenhoff KA, Ziman N, Markun LC, Racine CA, de Hemptinne C, Volz MM, Heath SL, Starr PA. 2017. Subthalamic nucleus deep brain stimulation in isolated dystonia: a 3-year follow-up study. Neurology 88:25-35. DOI: https://doi.org/10.1212/WNL.0000000000003451, PMID: 27903810

Parent A, Hazrati LN. 1995. Functional anatomy of the basal ganglia I the cortico-basal ganglia-thalamo-cortical loop. Brain Research Reviews 20:91-127. DOI: https://doi.org/10.1016/0165-0173(94)00007-C, PMID: 7711769

Parker JG, Marshall JD, Ahanonu B, Wu YW, Kim TH, Grewe BF, Zhang Y, Li JZ, Ding JB, Ehlers MD, Schnitzer MJ. 2018. Diametric neural ensemble dynamics in parkinsonian and dyskinetic states. Nature 557:177-182. DOI: https://doi.org/10.1038/s41586-018-0090-6, PMID: 29720658

Pedreira C, Martinez J, Ison MJ, Quian Quiroga R. 2012. How many neurons can we see with current spike sorting algorithms? Journal of Neuroscience Methods 211:58-65. DOI: https://doi.org/10.1016/j.jneumeth. 2012.07.010, PMID: 22841630

Penny W, Friston K, Ashburner J, Kiebel S, Nichols T. 2007. Statistical Parametric Mapping: The Analysis of Functional Brain Images. Academic Press.

Petryszyn S, Beaulieu JM, Parent A, Parent M. 2014. Distribution and morphological characteristics of striatal interneurons expressing calretinin in mice: a comparison with human and nonhuman primates. Journal of Chemical Neuroanatomy 59-60:51-61. DOI: https://doi.org/10.1016/j.jchemneu.2014.06.002, PMID: 24960462

Petryszyn S, Parent A, Parent M. 2018. The calretinin interneurons of the striatum: comparisons between rodents and primates under normal and pathological conditions. Journal of Neural Transmission 125:279-290. DOI: https://doi.org/10.1007/s00702-017-1687-x, PMID: 28168621

Piña-Fuentes D, Beudel M, Little S, van Zijl J, Elting JW, Oterdoom DLM, van Egmond ME, van Dijk JMC, Tijssen MAJ. 2018. Toward adaptive deep brain stimulation for dystonia. Neurosurgical Focus 45:E3. DOI: https://doi. org/10.3171/2018.5.FOCUS18155

Pisani A, Bernardi G, Ding J, Surmeier DJ. 2007. Re-emergence of striatal cholinergic interneurons in movement disorders. Trends in Neurosciences 30:545-553. DOI: https://doi.org/10.1016/j.tins.2007.07.008, PMID: 17 904652

Raz A, Feingold A, Zelanskaya V, Vaadia E, Bergman H. 1996. Neuronal synchronization of tonically active neurons in the striatum of normal and parkinsonian primates. Journal of Neurophysiology 76:2083-2088. DOI: https://doi.org/10.1152/jn.1996.76.3.2083, PMID: 8890317

Raz A, Vaadia E, Bergman H. 2000. Firing patterns and correlations of spontaneous discharge of pallidal neurons in the normal and the tremulous 1-methyl-4-phenyl-1,2,3,6-tetrahydropyridine vervet model of parkinsonism. The Journal of Neuroscience 20:8559-8571. DOI: https://doi.org/10.1523/JNEUROSCI.20-22-08559.2000, PMID: 11069964

Rey HG, Pedreira C, Quian Quiroga R. 2015. Past, present and future of spike sorting techniques. Brain Research Bulletin 119:106-117. DOI: https://doi.org/10.1016/j.brainresbull.2015.04.007, PMID: 25931392

Schönecker T, Kupsch A, Kühn AA, Schneider GH, Hoffmann KT. 2009. Automated optimization of subcortical cerebral MR imaging-atlas coregistration for improved postoperative electrode localization in deep brain stimulation. American Journal of Neuroradiology 30:1914-1921. DOI: https://doi.org/10.3174/ajnr.A1741, PMID: 19713324

Sharott A, Vinciati F, Nakamura KC, Magill PJ. 2017. A population of indirect pathway striatal projection neurons is selectively entrained to parkinsonian beta oscillations. The Journal of Neuroscience 37:9977-9998. DOI: https://doi.org/10.1523/JNEUROSCI.0658-17.2017, PMID: 28847810

Silberstein P, Kühn AA, Kupsch A, Trottenberg T, Krauss JK, Wöhrle JC, Mazzone P, Insola A, Di Lazzaro V, Oliviero A, Aziz T, Brown P. 2003. Patterning of globus pallidus local field potentials differs between 
Parkinson's disease and dystonia. Brain 126:2597-2608. DOI: https://doi.org/10.1093/brain/awg267, PMID: 12 937079

Singh A, Liang L, Kaneoke Y, Cao X, Papa SM. 2015. Dopamine regulates distinctively the activity patterns of striatal output neurons in advanced parkinsonian primates. Journal of Neurophysiology 113:1533-1544. DOI: https://doi.org/10.1152/jn.00910.2014, PMID: 25505120

Singh A, Mewes K, Gross RE, DeLong MR, Obeso JA, Papa SM. 2016. Human striatal recordings reveal abnormal discharge of projection neurons in Parkinson's disease. PNAS 113:9629-9634. DOI: https://doi.org/10.1073/ pnas.1606792113, PMID: 27503874

Singh A, Papa SM. 2019. Aberrant striatal oscillations after dopamine loss in parkinsonian non-human primates. bioRxiv. DOI: https://doi.org/10.1101/650770

Soares J, Kliem MA, Betarbet R, Greenamyre JT, Yamamoto B, Wichmann T. 2004. Role of external pallidal segment in primate parkinsonism: comparison of the effects of 1-methyl-4-phenyl-1,2,3,6-tetrahydropyridineinduced parkinsonism and lesions of the external pallidal segment. Journal of Neuroscience 24:6417-6426. DOI: https://doi.org/10.1523/JNEUROSCI.0836-04.2004, PMID: 15269251

Tewari A, Fremont R, Khodakhah K. 2017. It's not just the basal ganglia: cerebellum as a target for dystonia therapeutics. Movement Disorders 32:1537-1545. DOI: https://doi.org/10.1002/mds.27123, PMID: 28843013

Treu S, Strange B, Oxenford S, Neumann WJ, Kühn A, Li N, Horn A. 2020. Deep brain stimulation: imaging on a group level. Neurolmage 219:117018. DOI: https://doi.org/10.1016/j.neuroimage.2020.117018, PMID: 325056 98

Valsky D, Blackwell KT, Tamir I, Eitan R, Bergman H, Israel Z. 2020. Real-time machine learning classification of pallidal borders during deep brain stimulation surgery. Journal of Neural Engineering 17:016021. DOI: https:// doi.org/10.1088/1741-2552/ab53ac, PMID: 31675740

West AR, Grace AA. 2002. Opposite influences of endogenous dopamine D1 and D2 receptor activation on activity states and electrophysiological properties of striatal neurons: studies combining in vivo intracellular recordings and reverse microdialysis. The Journal of Neuroscience 22:294-304. DOI: https://doi.org/10.1523/ JNEUROSCI.22-01-00294.2002, PMID: 11756513

Wichmann T. 2018. Pathophysiologic basis of movement disorders. Progress in Neurological Surgery 33:13-24. DOI: https://doi.org/10.1159/000480718, PMID: 29332070

Wichmann T, DeLong MR. 2003. Pathophysiology of Parkinson's disease: the MPTP primate model of the human disorder. Annals of the New York Academy of Sciences 991:199-213. DOI: https://doi.org/10.1111/j.1749. 6632.2003.tb07477.x, PMID: 12846988

Wu T, Hallett M. 2013. The cerebellum in Parkinson's disease. Brain 136:696-709. DOI: https://doi.org/10.1093/ brain/aws360, PMID: 23404337

Yin HH, Knowlton BJ. 2006. The role of the basal ganglia in habit formation. Nature Reviews Neuroscience 7: 464-476. DOI: https://doi.org/10.1038/nrn1919, PMID: 16715055

Zaidel A, Spivak A, Grieb B, Bergman H, Israel Z. 2010. Subthalamic span of beta oscillations predicts deep brain stimulation efficacy for patients with Parkinson's disease. Brain 133:2007-2021. DOl: https://doi.org/10.1093/ brain/awq144, PMID: 20534648 


\section{DISCUSSION}

In this dissertation I designed machine learning methods that allow for accurate STN-ventral and striato-pallidal borders confirmation without needing an expert physiologist. Correlatively, the results help resolve a long-standing controversy on the nature of striatal dysfunction in PD. Below I discuss these findings as they emerged from my consecutive publications (Results IIII and Appendix I-II). Then, in the remainder of this discussion, I address each subject in more detail.

In the first study (Results I) we identified the neural signatures of the $\mathrm{SNr}$ and STN. To automatically discriminate between the STN and SNr during the DBS surgery, we designed a real-time HMM procedure. To understand the changes in the discharge rate and pattern of striatal projection neurons (SPNs) in PD and dystonia (Results III), we devised a method to delimit striato-pallidal borders (Results II). Contrary to long-standing assumptions, the third study (Results III) showed no evidence for spiny neuron dysfunction across a large data set collected from human PD and dystonic patients. Rather, the results suggest that spike sorting errors were the likely cause of the changes observed in neuronal firing rates reported in previous studies. These findings contribute to resolving the debate over the nature of striatal dysfunction in PD and suggest that changes in basal ganglia pathophysiology may arise through structures outside the striatum.

\section{A novel method to demarcate STN and SNr borders}

To date, automatic methods that use RMS and PSD values (Danish et al., 2008; J. H. Falkenberg et al., 2006; Moran et al., 2006; Novak et al., 2007; Zaidel et al., 2009) are successful in identifying STN-white matter (STN-WM) transitions, but are not as good for the direct STN-SNr transition. By adding a new feature to the machine-learning procedure; namely, the ratio of high frequency $(100-150 \mathrm{~Hz})$ power to low frequency $(5-25 \mathrm{~Hz})$ power, we developed an innovative method (Results I) that can detect the transition between STN and SNr. We used a SVM procedure to verify that the $100-150 \mathrm{~Hz} / 5-25 \mathrm{~Hz}$ power ratio reliably discriminated between STN and SNr populations. Then, we utilized an HMM procedure using the MER features, together with the trajectory history to detect the STN exit either to the white matter (WM) or SNr. This HMM procedure can also deal with heterogeneous variation in trajectories, since some transitions go from the VMNR STN to the SNr and others from the white matter above the STN to the SNr.

Because this real-time detection algorithm is used in the operating room it needs to function on novel data without being continually adjusted. We demonstrated that the HMM procedure can successfully process completely unknown data. We used 73 new trajectories (from 51 patients, all trajectories included both $\mathrm{STN}$ and $\mathrm{SNr}$ ) to evaluate the HMM procedure. The STN OUT location error for both the STN-SNr and the STN-WM exhibited better means and standard deviations than the previous HMM procedure (Zaidel et al., 2009) or the Bayesian method (Moran et al., 2006). It only took $99 \mathrm{~ms}$ in real time (Intel Core I5 2410M processor with 4GB RAM) to process a new trajectory and decide whether it was the STN or not, making this a practical method for use during DBS surgery. 


\section{Oscillations in awake versus light anesthesia dystonia classes}

Several recent studies have reported differences in pallidal firing rates between dystonia classes under volatile anesthetics (McClelland et al., 2016; Welter et al., 2015). In Results II, we extended previous work by comparing the spectral oscillatory changes in neural activity within the striatum, GPe and GPi between awake and lightly anesthetized genetic and non-genetic dystonia classes. Using the $\mathrm{L}_{1}$-distance measure, we determined that the awake and the light anesthesia (0.3-0.6 MAC range) dystonia classes could be merged. Thus, although anesthesia may reduce pallidal neuronal oscillations across all dystonia classes (Hutchison et al., 2003; Venkatraghavan et al., 2016), detectable relative differences in neural oscillations between genetic dystonia and non-genetic classes are preserved.

A growing body of evidence suggests that anesthetics induce oscillations that alter or disrupt the normal brain's information processing (Cimenser et al., 2011; Purdon et al., 2013). Because the cortex is the major afferent to the striatum (Kemp and Powell, 1970), profound changes in neural activity in the cortex, which are reflected in major changes in the scalp electroencephalogram (EEG), may be transmitted to the striatum. Our MER-derived results revealed that alpha oscillations in the striatum under light anesthesia were consistent with findings showing an increase in oscillatory EEG power in the alpha frequency range under isoflurane or sevoflurane anesthesia (Purdon et al., 2015). Even though striatal neurons send inhibitory projections to the GPe and GPi (Haber, 2003), we found that striatal neurons did not propagate these alpha oscillation to them. This may be explained by studies in non-human primates indicating that the output activity of the pallidum (or basal-ganglia downstream structures) does not reflect striatal neural activity (Deffains et al., 2016a). This suggests that abnormal oscillatory activity within striatum, under light anesthesia, did not propagate to the GPe and GPi. Another possibility is that the oscillatory activity in the different domains of the striatum (e.g., ventral and dorsal striatum) was not the same. We may therefore have recorded the neural activity from those parts of the GPe and GPi that receive information from domains of the striatum that are not involved in alpha oscillations.

\section{Differences in striato-GPe-GPi oscillatory activity between the PD, genetic dystonia and non-genetic dystonia classes}

In Results II, by comparing the spatial distribution of the oscillatory neural activity within the striatum, GPe and GPi across the three classes of the disease, we found that pathological betaoscillations comprised a continuous stretch within the GPi PD. This is unlike the GPe PD that forms gradient beta-oscillations as a function of the distance from the ventral border. We then found that the spatial distribution of low-frequency activity for both the GPe and GPi was nearly identical across the genetic and non-genetic dystonia patients. In contrast, the GPe and GPi in PD exhibited both tremor $(4-5 \mathrm{~Hz})$ and beta frequency oscillations.

These differences in striato-GPe-GPi oscillatory activity between the PD, genetic dystonia and non-genetic dystonia classes could be used in the future as electrophysiological target-specific biomarkers to optimize therapy in adaptive DBS control systems (Meidahl et al., 2017; Wang et al., 2018). 


\section{Real-time machine learning for striato-pallidal localization}

In Appendix II, we showed that NRMS values calculated from the MER segments were effective in detecting the striatum-GPe and GPi-exit borders because of their increase and decrease in the NRMS. In contrast, detection of the GPe-GPi borders was more difficult, since often there was no sharp increase or decrease in NRMS between these structures. However, visual inspection of the mean power spectra did reveal differences between spectra in the 3$300 \mathrm{~Hz}$ domain. To determine quantitatively which part of the spectral domain enables the best discrimination between regions, we calculated the absolute difference in the mean power spectra. We then examined eight different frequency bands (approximately logarithmically equally distributed) along the frequency axis (Appendix II). Frequency bands at 3-30 Hz, 8-35 $\mathrm{Hz}, 30-95 \mathrm{~Hz}$ and 180-245 Hz provided the greatest discrimination for the striatum-GPe and GPe-GPi transitions. We used these frequency bands to calculate the input features of the HMM.

In Results II, we designed an HMM to demarcate the striato-pallidal borders. We found that the HMM varied in its ability to predict GPe- and GPi- entry and exit. Whereas the striatumGPe and GPi-exit were usually clear and characterized by a distinguishable rise and fall of the NRMS, respectively, the GPe-GPi transitions were harder to detect in some trajectories. The GPe is separated from the GPi by a thin layer of myelinated fibers called the medial medullar lamina (Lanciego et al., 2012). The GPe-GPi transition can therefore be detected by a drop in previous neuronal activity and the recording of border cells in its vicinity. However, the transition from the GPe to GPi was not always clear to the expert or to the HMM algorithm. This may have been due in part to the sparsely distributed neurons along the pallidum and the variability in the firing characteristics between the GPe and GPi in the MER dataset (Schiff et al., 2002). Clinically, GPe-GPi boundary reliability is probably not very important. This is because, for accurate DBS lead placement, the critical boundary is the GPi-exit border where the PD algorithm achieved $83 \%$ accuracy (on the test data) within a $0.3 \mathrm{~mm}$ distance (an error considered "acceptable" by our team). Localization of the striato-pallidal borders can help the neurosurgeon decide which MER track to implant, when multiple electrodes are used for MER, and when implanting the macroelectrode at the optimal depth.

Further research is necessary to evaluate performance in different clinical settings, in order to validate this algorithm across the spatial distributions of neural activity encountered along the trajectory in the typical operating room setting. The HMM is agnostic to the type of raw data used and can be implemented easily in existing MER devices. The ability to classify striatopallidal regions in real-time using our classification algorithm may shorten DBS surgery considerably. The machine learning algorithm approach considers all available example trajectories equally and finds transitions between nuclei that a human researcher may miss for example, due to bias towards a given interpretation. Our method allows accurate GPi confirmation without needing the input of an expert physiologist.

\section{Discharge rate and pattern of the striatal projection neurons in Parkinson's disease and dystonia}

In Results III, we helped to resolve the long-standing controversy about the nature of striatal dysfunction in PD. The prevailing theory of basal ganglia dysfunction in PD is that it originates from alterations in the firing rates or patterns of D2-expressing spiny projection neurons in the striatum. Contrary to these assumptions we found no evidence for spiny neuron dysfunction, 
and that spike sorting errors were the likely cause of the changes observed in neuronal firing rates in previous studies. The findings in Results III suggest that the changes in basal ganglia pathophysiology most probably arose in through structures outside the striatum.

The methods in Results III go beyond previous reports by utilizing a machine learning algorithm (Results II) to automatically detect the striato-pallidal border, and by applying objective methods for the identification of units and the quantification of their isolation quality and stationarity. Using these methods, we found that the firing rate of the SPNs of both the PD and dystonic patients was extremely sensitive to the quality of the isolation of the units. Specifically, we found a negative correlation between striatal firing rate and isolation score. The negative correlation between the discharge rate of the detected units and their isolation quality revealed that the spike detection and sorting algorithm used in our study and/or the physical properties of the striatal neurons tended to erroneously classify noise events as spikes (false positives) rather than missing real spikes (false negatives). The amplitude of the extracellular action potentials (or the signal-to-noise ratio) was mainly sensitive to the impedance of the microelectrode and the distance between the recorded neurons and the microelectrode, and was not related to the firing rate of the neurons. This thus ruled out the possibility that fast firing striatal neurons were less frequently well-isolated and underrepresented in the high-score bins. We found that by removing the non-stationary units also reduced the striatal firing rate of SPNs in both the PD and dystonic patients. This may indicate that the inclusion of non-stationary ("injured") units can also erroneously increase the striatal firing rate in PD and dystonic patients. The difference in results between our study and Singh et al., (2016) may be due to: (i) a difference between machine learning detection vs. manual electrophysiologist detection of the striato-pallidal border, (ii) different spike sorting and detection methods, (iii) the use of algorithms for the quantification of the isolation quality and stationarity of the units vs. manual electrophysiologist decision-making, and (iv) different recording techniques (microelectrode type, step size and regular spaced recordings vs. manual search of the recording sites).

Overall, our results on patients extend a previous study in the MPTP NHP model of PD (Deffains et al., 2016b) and studies in the 6-OHDA rodent model (Ketzef et al., 2017; Maltese et al., 2019b) and demonstrate that abnormal activity along both the direct and indirect pathways of the BG network is not caused by drastic changes in spontaneous SPN spiking activity. Recently, Maltese et al. (2019) reported abnormal recruitment (e.g., during behavior) of D1 and D2 SPNs (which are mostly silent and at rest in the healthy condition) in parkinsonism that resulted in an aberrant net balance of striatal outputs, although there were no changes in the firing rate of the individual neurons. Our experimental approach (i.e., extracellular recordings in awake patients in the operating room) does not allow us to estimate the sample size of the activated neurons. Moreover, we did not have a control condition and recordings were not performed during behavior, to minimize surgical procedure time. Therefore, further experiments in primates are needed to validate or refute this possibility. It is likely that these small to moderate changes in spontaneous SPN discharge will be amplified by BG downstream structures, thus leading to the clinical symptoms of PD and possibly of dystonia. 


\section{APPENDICES}

Supplementary material for article in Results I

\section{Discrimination matrix of the STN-SNr}

Table S1: The frequency dependence of the STN-SNr discrimination matrix.

\begin{tabular}{|c|c|c|c|c|c|}
\hline \multirow{2}{*}{\multicolumn{2}{|c|}{$\begin{array}{l}\text { Discrimination } \\
\text { matrix }\end{array}$}} & \multicolumn{4}{|c|}{ Width of each band [Hz] } \\
\hline & & $10 \mathrm{~Hz}$ & $20 \mathrm{~Hz}$ & $50 \mathrm{~Hz}$ & $100 \mathrm{~Hz}$ \\
\hline \multirow{4}{*}{ 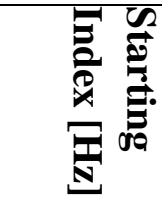 } & $5 \mathrm{~Hz}$ & 4.28 & 5.30 & 1.71 & 0.04 \\
\hline & $50 \mathrm{~Hz}$ & NA & 2.62 & 2.02 & 2.41 \\
\hline & $100 \mathrm{~Hz}$ & NA & NA & 15.55 & 4.13 \\
\hline & $200 \mathrm{~Hz}$ & NA & NA & NA & 1.99 \\
\hline
\end{tabular}

Values depict the discrimination between STN and SNr of 10 approximately logarithmically spaced bands throughout the 5-300 Hz range of the power spectra. The band starts at the starting index and has a specified width (e.g., top left box is the $5-15 \mathrm{~Hz}$ band). The starting index of the band width was simultaneously increased (e.g., for a starting index $=200 \mathrm{~Hz}$, we only tested a width of $100 \mathrm{~Hz}, 200-300 \mathrm{~Hz}$ band). For each band the mean power $\left(\mathrm{V}^{2} / \mathrm{Hz}\right)$ for each MER was calculated. Then, for each band, the absolute value of the difference in the mean power between the STN and the SNr was obtained. Finally, the results were normalized by the square root of the sum of the variances of the STN and the $\mathrm{SNr}$ to yield the discrimination index $\left(\mathrm{V}^{2} / \mathrm{Hz}\right)$. Maximal STN-SNr discrimination was obtained in two different frequency bands: high frequency $(100-150 \mathrm{~Hz})$ with a value of 15.55 , and low frequency $(5-25 \mathrm{~Hz})$ with a value of 5.3.

\section{Support vector machine discrimination performance}

Table S2: SVM discrimination performance

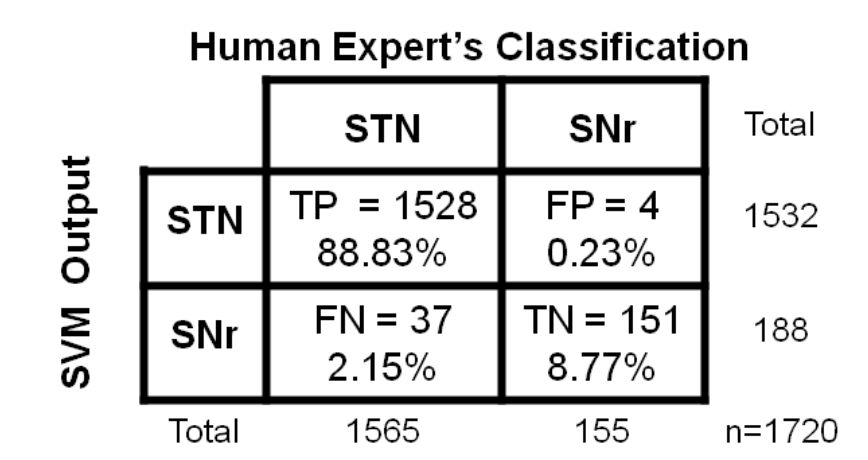

The discrimination performance of the SVM classifier for the training dataset. The NRMS and the $100-150 \mathrm{~Hz} / 5-25 \mathrm{~Hz}$ Power Ratio were evaluated by the 10 -fold cross validation method and are presented as an error matrix. TP, true positive; TN, true negative; FN, false negative; FP, false positive. 


\section{The Hidden Markov Model}

For the HMM procedure, a trajectory is a sequence of states, and at any depth along the trajectory the electrode exists in one of a finite set of states (functionally discrete state model, presented in Figure 1C).

1. White matter before STN

2. Dorso lateral oscillatory region (DLOR) STN

3. Ventral medial non oscillatory region (VMNR) STN

4. STN exit
a. White matter between STN and SNr
b. or $\mathrm{SNr}$

A typical trajectory state sequence proceeds through the first three states consecutively and then to the white matter between the STN and SNr (solid line in Figure 1C). However, not all trajectories have clearly defined STN-WM transitions; hence, a trajectory can enter the $\mathrm{SNr}$ immediately after the STN (dotted line). In addition, a trajectory can start in the white matter and enter the VMNR or go directly into the $\mathrm{SNr}$ (dashed lines) without passing through the STN.

In the HMM, transitions between states take place according to (1) a fixed probability (i.e., transition matrix and emission matrix) depending solely on the state of the electrode at the depth immediately preceding the current state and (2) a probability value calculated from the current MER. Note that we used a feed-forward HMM procedure; i.e., as a sequence progresses, it is possible to remain in the same state, but it is not possible to return to a previous state (e.g., from the SNr to the STN state). 
Table S3: The HMM transition and emission matrices.

A

\begin{tabular}{|c|c|c|c|c|c|}
\hline \multicolumn{2}{|c|}{ Transition matrix } & \multicolumn{4}{|l|}{ To } \\
\hline & & State-1 & State-2 & State-3 & State-4 \\
\hline \multirow{4}{*}{$\begin{array}{l}\overrightarrow{3} \\
\hat{0}\end{array}$} & State-1 & 0.9510 & 0.0280 & 0.0103 & 0.0107 \\
\hline & State-2 & 0 & 0.9601 & 0.0397 & 0.0002 \\
\hline & State-3 & 0 & 0 & 0.9617 & 0.0383 \\
\hline & State-4 & 0 & 0 & 0 & 1.0000 \\
\hline
\end{tabular}

B

\begin{tabular}{|c|c|c|c|c|c|c|c|c|}
\hline \multirow{4}{*}{\multicolumn{2}{|c|}{$\begin{array}{l}\text { Emission } \\
\text { matrix }\end{array}$}} & \multicolumn{7}{|c|}{ Observations } \\
\hline & & \multirow{3}{*}{$\begin{array}{l}\text { Low- } \\
\text { NRMS }\end{array}$} & \multirow{3}{*}{$\begin{array}{l}\text { Interme } \\
\text { diate- } \\
\text { NRMS }\end{array}$} & \multicolumn{5}{|c|}{ high-NRMS } \\
\hline & & & & \multicolumn{2}{|c|}{ High mean beta } & \multicolumn{2}{|c|}{ Low mean beta } & \multirow{2}{*}{$\begin{array}{l}\text { High to } \\
\text { Low } \\
\text { Power } \\
\text { Ratio }\end{array}$} \\
\hline & & & & $\begin{array}{l}\text { High } \\
\text { max } \\
\text { beta }\end{array}$ & $\begin{array}{l}\text { Low } \\
\text { max } \\
\text { beta }\end{array}$ & $\begin{array}{l}\text { High } \\
\text { max } \\
\text { beta }\end{array}$ & $\begin{array}{l}\text { Low } \\
\text { max } \\
\text { beta }\end{array}$ & \\
\hline \multirow{4}{*}{$\frac{\mathscr{0}}{\overrightarrow{0}}$} & State-1 & 0.8685 & 0.0261 & 0.0348 & 0.0044 & 0.0039 & 0.0254 & 0.0365 \\
\hline & State-2 & 0.3056 & 0.0263 & 0.3786 & 0.0434 & 0.0336 & 0.1755 & 0.0367 \\
\hline & State-3 & 0.3167 & 0.0534 & 0.0769 & 0.0239 & 0.0780 & 0.3694 & 0.0814 \\
\hline & State-4 & 0.5382 & 0.1881 & 0.0130 & 0.0012 & 0.0207 & 0.1054 & 0.1330 \\
\hline
\end{tabular}

(A) The HMM transition matrix. Values depict the probability of a transition between states (or remaining in the current state) for each step in the sequence. The states are defined as follows: 1 - WM before the STN; 2 - in the dorsolateral oscillatory region (DLOR) of the STN; 3 - in the ventro medial non-oscillatory (VMNR) of the STN; 4-STN exit (STN-SNr or STNWM). (B) The HMM emission matrix. Values depict the probability of each observation given the state. The states are defined as in A. The seven columns correspond to the seven observations detailed in the table header as follows:

- All MERs with NRMS <1.25 (threshold 1); i.e., below a 25\% increase from the NRMS baseline (which is equal to 1 due to the normalization) were clustered together (LowNRMS observation). 
- The mean deviation from threshold 1 (i.e. NRMS - 1.25) of the remaining MERs was calculated. Threshold 2 was defined as threshold 1 plus $25 \%$ of the calculated mean deviation. MERs with a NRMS between threshold 1 and threshold 2 were clustered together (Intermediate-NRMS observation), and MERs with NRMS> threshold 2 were further divided according to their (maximum and mean) beta $(13-30 \mathrm{~Hz})$ oscillatory activity (above or below the median), resulting in four more (high-NRMS) observations.

- A high to low power ratio observation was calculated for all the MERs and was independent of the NRMS observations. 
PhD Thesis, Dan Valsky

Table S4: Patient characteristics

\begin{tabular}{|c|c|c|c|c|c|c|}
\hline \multirow[b]{2}{*}{ Patient No. } & \multirow[b]{2}{*}{ Sex } & \multirow[b]{2}{*}{$\begin{array}{c}\begin{array}{c}\text { Age } \\
\text { (years) }\end{array} \\
\end{array}$} & \multirow[b]{2}{*}{$\begin{array}{c}\begin{array}{c}\text { Disease duration } \\
\text { (years) }\end{array} \\
\end{array}$} & & & \\
\hline & & & & UPDRS 3 (OFF) & UPDRS 3 (On) & LED \\
\hline 1 & $\mathrm{~m}$ & 69 & 8 & 55 & 13 & 1500 \\
\hline 2 & $\mathrm{f}$ & 61 & 6 & 64 & 32 & N/A \\
\hline 3 & $\mathrm{~m}$ & 66 & 5 & 51 & 22 & 750 \\
\hline 4 & f & 67 & 5 & 53 & 29 & 1000 \\
\hline 5 & $\mathrm{~m}$ & 67 & 26 & 55 & 24 & 550 \\
\hline 6 & $\mathrm{~m}$ & 59 & 8 & 48 & 13 & 570 \\
\hline 7 & $\mathrm{~m}$ & 52 & 7 & 38 & 12 & 540 \\
\hline 8 & $\mathrm{~m}$ & 64 & 8 & 41 & 6 & 825 \\
\hline 9 & $\mathrm{~m}$ & 60 & 5 & 39 & 15 & 1580 \\
\hline 10 & $\mathrm{~m}$ & 42 & 25 & 77 & 18 & 500 \\
\hline 11 & $\mathrm{~m}$ & 60 & 7 & 85 & 30 & 870 \\
\hline 12 & $\mathrm{~m}$ & 75 & 4 & 39 & 28 & 562.5 \\
\hline 13 & $\mathrm{~m}$ & 69 & 7 & 45 & 17 & 750 \\
\hline 14 & $\mathrm{f}$ & 74 & 13 & 33 & 10 & 830 \\
\hline 15 & $\mathrm{~m}$ & 63 & 6 & 59 & 18 & 810 \\
\hline 16 & $\mathrm{~m}$ & 65 & 8 & 48 & 12 & 750 \\
\hline 17 & $\mathrm{f}$ & 67 & 15 & 35 & 11 & 1147.5 \\
\hline 18 & $\mathrm{~m}$ & 59 & 8 & 36 & 10 & 900 \\
\hline 19 & $\mathrm{f}$ & 57 & 8 & 48 & 19 & 680 \\
\hline 20 & $\mathrm{~m}$ & 53 & 3 & 49 & 6 & 200 \\
\hline 21 & $\mathrm{~m}$ & 64 & 4 & 56 & 34 & 850 \\
\hline 22 & $\mathrm{~m}$ & 74 & 16 & 57 & 31 & 750 \\
\hline 23 & $\mathrm{f}$ & 71 & 22 & 51 & 35 & 2000 \\
\hline 24 & $\mathrm{f}$ & 66 & 4 & 46 & 16 & 750 \\
\hline 25 & $\mathrm{~m}$ & 50 & 10 & 47 & 35 & 93.7 \\
\hline 26 & $\mathrm{~m}$ & 64 & 9 & 69 & 48 & 900 \\
\hline 27 & $\mathrm{~m}$ & 62 & 30 & 56 & 22 & 1120 \\
\hline 28 & $\mathrm{~m}$ & 66 & 10 & 63 & 11 & 600 \\
\hline 29 & $\mathrm{~m}$ & 61 & 7 & 32 & 7 & 750 \\
\hline 30 & $\mathrm{f}$ & 59 & 13 & 75 & 19 & 1000 \\
\hline 31 & $\mathrm{~m}$ & 54 & 9 & 75 & 41 & 500 \\
\hline 32 & $\mathrm{~m}$ & 64 & 12 & 35 & 2 & 875 \\
\hline 33 & $\mathrm{~m}$ & 50 & 20 & 53 & 39 & N/A \\
\hline 34 & $\mathrm{f}$ & 73 & 5 & 73 & 42 & 1250 \\
\hline 35 & $\mathrm{~m}$ & 57 & 7 & 78 & 37 & 1370 \\
\hline 36 & $\mathrm{~m}$ & 69 & 8 & 50 & 10 & 1140 \\
\hline 37 & $\mathrm{~m}$ & 66 & 9 & 34 & 14 & 1000 \\
\hline 38 & $\mathrm{~m}$ & 58 & 10 & 47 & 13 & N/A \\
\hline 39 & $\mathrm{~m}$ & 58 & 12 & 47 & 11 & 1120 \\
\hline 40 & $\mathrm{f}$ & 62 & 14 & 37 & 9 & 1000 \\
\hline 41 & $\mathrm{~m}$ & 76 & 7 & 32 & 19 & 1120 \\
\hline 42 & $\mathrm{f}$ & 62 & 13 & 32 & 6 & 925 \\
\hline 43 & $\mathrm{~m}$ & 70 & 10 & N/A & N/A & 950 \\
\hline 44 & $\mathrm{f}$ & 59 & 10 & 50 & 14 & 455 \\
\hline 45 & $\mathrm{f}$ & 61 & 10 & N/A & N/A & 1000 \\
\hline 46 & $\mathrm{~m}$ & 66 & 10 & 68 & 27 & 1250 \\
\hline 47 & $\mathrm{~m}$ & 73 & 20 & 60 & 16 & 1496 \\
\hline 48 & $\mathrm{~m}$ & 60 & 8 & 46 & 21 & 500 \\
\hline 49 & f & 64 & 9 & 21 & 4 & 917.5 \\
\hline 50 & $\mathrm{~m}$ & 54 & 5 & 86 & 42 & 500 \\
\hline 51 & $\mathrm{~m}$ & 59 & 4 & 64 & 26 & 1000 \\
\hline 52 & $\mathrm{f}$ & 60 & 8 & 44 & 12 & 975 \\
\hline 53 & $\mathrm{~m}$ & 78 & 10 & N/A & N/A & 2075 \\
\hline 54 & $\mathrm{~m}$ & 62 & 17 & 72 & 22 & 1550 \\
\hline 55 & f & 70 & 13 & 41 & 13 & 400 \\
\hline 56 & f & 69 & 9 & 33 & 6 & 1875 \\
\hline 57 & $\mathrm{~m}$ & 53 & 9 & 41 & 10 & 375 \\
\hline 58 & $\mathrm{~m}$ & 72 & 12 & N/A & N/A & 1125 \\
\hline 59 & $\mathrm{f}$ & 50 & 8 & N/A & N/A & 550 \\
\hline 60 & f & 45 & 9 & 82 & 55 & 700 \\
\hline 61 & $\mathrm{~m}$ & 59 & 9 & 42 & 14 & 150 \\
\hline 62 & $\mathrm{~m}$ & 62 & 12 & 55 & 19 & 1005 \\
\hline 63 & $\mathrm{~m}$ & 62 & 4 & 63 & 17 & 1080 \\
\hline 64 & $\mathrm{~m}$ & 52 & 8 & 27 & 3 & 510 \\
\hline 65 & $\mathrm{f}$ & 52 & 10 & 35 & 9 & 770 \\
\hline 66 & $\mathrm{f}$ & 66 & 15 & 48 & 13 & 1540 \\
\hline 67 & $\mathrm{~m}$ & 49 & 13 & 63 & 18 & 660 \\
\hline 68 & f & 48 & 18 & 47 & 20 & 300 \\
\hline 69 & $\mathrm{~m}$ & 60 & 11 & 56 & 14 & 850 \\
\hline 70 & $\mathrm{f}$ & 39 & 10 & 47 & 16 & 512.5 \\
\hline 71 & $\mathrm{~m}$ & 61 & 11 & 44 & 10 & 500 \\
\hline 72 & $\mathrm{f}$ & 50 & 25 & 50 & 17 & 400 \\
\hline 73 & $\mathrm{f}$ & 72 & 7 & 60 & 24 & 100 \\
\hline 74 & $\mathrm{f}$ & 51 & 4 & 20 & 7 & N/A \\
\hline 75 & $\mathrm{~m}$ & 73 & 8 & 55 & 27 & 562.5 \\
\hline 76 & $\mathrm{~m}$ & 66 & 6 & 75 & 38 & 450 \\
\hline 77 & f & 73 & 10 & 45 & 18 & 1750 \\
\hline 78 & $\mathrm{f}$ & 62 & 20 & 56 & 9 & 517.5 \\
\hline 79 & $\mathrm{~m}$ & 74 & 6 & 61 & 31 & 750 \\
\hline 80 & $\mathrm{f}$ & 67 & 8 & 48 & 3 & 250 \\
\hline 81 & $\mathrm{~m}$ & 72 & 6 & 42 & 33 & 640 \\
\hline $\begin{array}{c}\text { sum } / \\
\text { mean } \pm \mathrm{SD}\end{array}$ & $\begin{array}{c}52 \mathrm{~m} \\
29 \mathrm{f}\end{array}$ & $62.1 \pm 8.3$ & $10.3 \pm 5.5$ & $51.1 \pm 14.8$ & $19.4 \pm 11.51$ & $849.6 \pm 425$ \\
\hline
\end{tabular}

N/A : Not Available

UPDRS 3 (OFF/ON medication) : clinician-scored motor evaluation (range 0-108)

LED : Levopoda Equivalent Dose 


\section{MRI image that shows typical anatomical targeting}

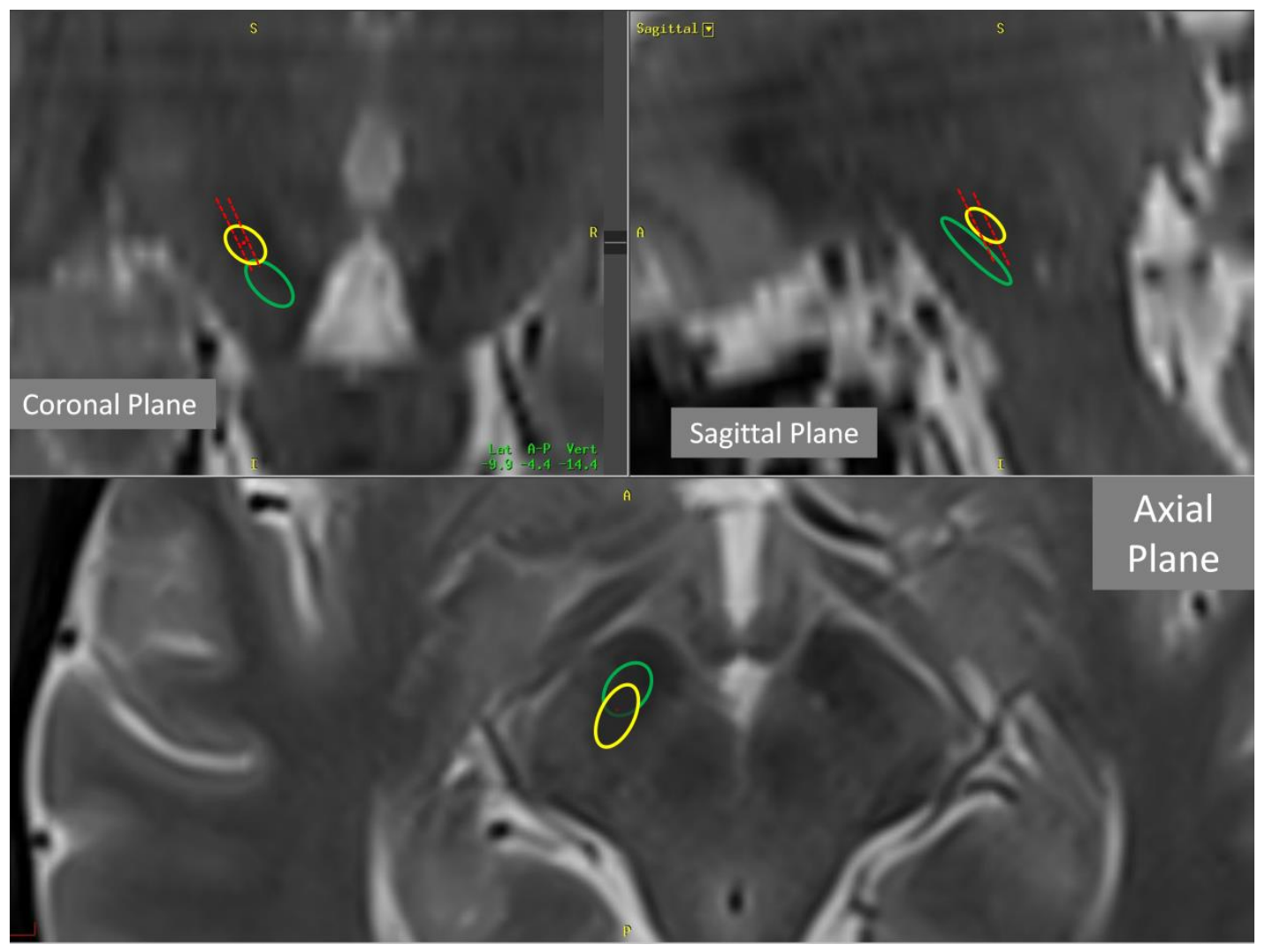

T2 weighted Axial MR image $6 \mathrm{~mm}$ below the ACPC plane with coronal and sagittal reconstructions. The STN is shown approximately outlined in yellow, the $\mathrm{SNr}$ is shown approximately outlined in green. The dotted red lines represent possible examples of MER tracks that traverse the STN and may enter the SNR. Although the target is the dorsolateral part of the STN, we aimed to have as long a trajectory as possible traversing the STN through the target point. 
Supplementary material for article in Results II

\section{Neural Datasets}

Supplementary Table S1a: Patient demographics and MER dataset for the five classes

\begin{tabular}{|c|c|c|c|}
\hline $\mathbf{A}$ & $\begin{array}{c}\text { Number of } \\
\text { Patients }\end{array}$ & $\begin{array}{l}\text { Number of } \\
\text { Trajectories }\end{array}$ & $\begin{array}{c}\text { Number of } \\
\text { MER's }\end{array}$ \\
\hline Parkinson's Disease & 10 & 33 & 2884 \\
\hline Dystonia Non-Genetic awake & 8 & 29 & 2380 \\
\hline Dystonia Non-Genetic GA & 9 & 18 & 2202 \\
\hline Dystonia Genetic awake & 5 & 14 & 1752 \\
\hline Dystonia Genetic GA & 10 & 22 & 2556 \\
\hline Total & 42 & 116 & 11774 \\
\hline B & Str & GPe & GPi \\
\hline Parkinson's Disease & 571 & 1306 & 1007 \\
\hline Dystonia Non-Genetic awake & 499 & 974 & 907 \\
\hline Dystonia Non-Genetic GA & 539 & 823 & 840 \\
\hline Dystonia Genetic awake & 377 & 659 & 716 \\
\hline Dystonia Genetic GA & 571 & 939 & 1046 \\
\hline Total & 2557 & 4701 & 4516 \\
\hline
\end{tabular}

A) The dataset included trajectories from 42 patients consisting of 11,774 MERs in five classes of the disease: awake Parkinson's disease patients, awake and lightly anesthetized genetic and non-genetic dystonia patients. B) MER dataset for the five classes of the disease in the three regions: Striatum (Str), GPe and GPi.

Supplementary Table S1b: Patient demographics and MER dataset for three classes

\begin{tabular}{|c|c|c|c|}
\hline A & $\begin{array}{c}\text { Number of } \\
\text { Patients }\end{array}$ & $\begin{array}{l}\text { Number of } \\
\text { Trajectories }\end{array}$ & $\begin{array}{c}\text { Number of } \\
\text { MER's }\end{array}$ \\
\hline Parkinson's Disease & 10 & 33 & 2884 \\
\hline Dystonia Non-Genetic & 17 & 47 & 4582 \\
\hline Dystonia Genetic & 15 & 36 & 4308 \\
\hline Total & 42 & 116 & 11774 \\
\hline B & Str & $\mathrm{GPe}$ & $\mathrm{GPi}$ \\
\hline Parkinson's Disease & 571 & 1306 & 1007 \\
\hline Dystonia Non-Genetic & 1038 & 1797 & 1747 \\
\hline Dystonia Genetic & 948 & 1598 & 1762 \\
\hline Total & 2557 & 4701 & 4516 \\
\hline
\end{tabular}


A) Same as in S1a, but after merging of the awake and light anesthesia dystonia classes. We used these three classes of the disease (i.e., PD, non-genetic and genetic dystonia) for our working system. B) MER dataset of Striatum (Str), GPe and GPi

\section{Power spectral density (PSD)}

The PSD was calculated from the mean-subtracted absolute value of the analog signal (Moran et al., 2008; Moran and Bar-Gad, 2010). This absolute procedure was needed to expose the frequency band of interest (below $300 \mathrm{~Hz}$ ) since the original analog data were band-pass filtered at 300-9000 Hz (the $300 \mathrm{~Hz}$ cut-off was used because of operating room constraints). The average power spectral density was calculated for each trace using Welch's method, with a 3 second Hamming window (50\% overlap) and a spectral resolution of $1 / 3 \mathrm{~Hz}$ (sampling frequency $44 \mathrm{kHz}$, number of discrete Fourier transform points =132,000). Values within $2 \mathrm{~Hz}$ of the $50 \mathrm{~Hz}$ power supply artifacts and their 100 and $150 \mathrm{~Hz}$ harmonics were removed and interpolated from the surrounding values. To normalize the PSD we divided the PSD by the total power of the signal from 0 to $22 \mathrm{KHz}$ (excluding PSD values within $2 \mathrm{~Hz}$ of the $50 \mathrm{~Hz}$ power supply artifacts and their 100 and $150 \mathrm{~Hz}$ harmonics) creating a relative PSD. This normalization revealed the relative power across frequencies. 


\section{Discrimination matrices of the Str-GPe and GPe-GPi}

Table S2: The frequency dependence of the Str-GPe and GPe-GPi discrimination matrices.

A

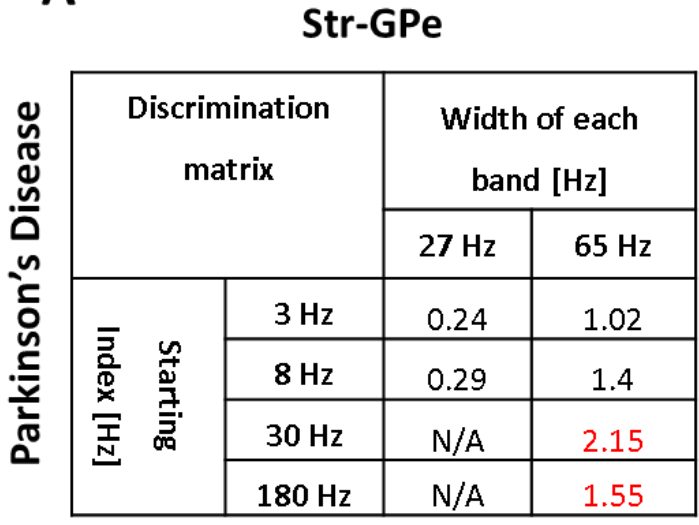

\begin{tabular}{|c|c|c|c|}
\hline \multicolumn{2}{|c|}{$\begin{array}{c}\text { Discrimination } \\
\text { matrix }\end{array}$} & \multicolumn{2}{|c|}{$\begin{array}{c}\text { Width of each } \\
\text { band }[\mathrm{Hz}]\end{array}$} \\
\hline & & $27 \mathrm{~Hz}$ & $65 \mathrm{~Hz}$ \\
\hline \multirow{4}{*}{$\frac{n}{2}$} & $3 \mathrm{~Hz}$ & 2.84 & 2.52 \\
\hline & $8 \mathrm{~Hz}$ & 3.00 & 2.52 \\
\hline & $30 \mathrm{~Hz}$ & $\mathrm{~N} / \mathrm{A}$ & 1.69 \\
\hline & $180 \mathrm{~Hz}$ & $\mathrm{~N} / \mathrm{A}$ & 1.35 \\
\hline
\end{tabular}

\begin{tabular}{|c|c|c|c|}
\hline \multicolumn{2}{|c|}{$\begin{array}{c}\text { Discrimination } \\
\text { matrix }\end{array}$} & \multicolumn{2}{|c|}{$\begin{array}{c}\text { Width of each } \\
\text { band [Hz] }\end{array}$} \\
\hline & & $27 \mathrm{~Hz}$ & $65 \mathrm{~Hz}$ \\
\hline \multirow{4}{*}{ 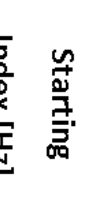 } & $3 \mathrm{~Hz}$ & 3.57 & 2.63 \\
\hline & $8 \mathrm{~Hz}$ & 3.49 & 2.40 \\
\hline & $30 \mathrm{~Hz}$ & N/A & 0.93 \\
\hline & $180 \mathrm{~Hz}$ & $\mathrm{~N} / \mathrm{A}$ & 1.02 \\
\hline
\end{tabular}

B GPe-GPi

\begin{tabular}{|c|c|c|c|}
\hline \multicolumn{2}{|c|}{$\begin{array}{l}\text { Discrimination } \\
\text { matrix }\end{array}$} & \multicolumn{2}{|c|}{$\begin{array}{c}\text { Width of each } \\
\text { band }[\mathrm{Hz}]\end{array}$} \\
\hline & & $27 \mathrm{~Hz}$ & $65 \mathrm{~Hz}$ \\
\hline \multirow{4}{*}{ 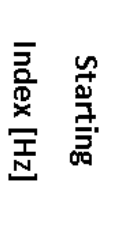 } & $3 \mathrm{~Hz}$ & 0.01 & 0.76 \\
\hline & $8 \mathrm{~Hz}$ & 0.38 & 0.85 \\
\hline & $30 \mathrm{~Hz}$ & $\mathrm{~N} / \mathrm{A}$ & 1.21 \\
\hline & $180 \mathrm{~Hz}$ & N/A & 1.46 \\
\hline
\end{tabular}

\begin{tabular}{|c|c|c|c|}
\hline \multicolumn{2}{|c|}{$\begin{array}{l}\text { Discrimination } \\
\text { matrix }\end{array}$} & \multicolumn{2}{|c|}{$\begin{array}{c}\text { Width of each } \\
\text { band }[\mathrm{Hz}]\end{array}$} \\
\hline & & $27 \mathrm{~Hz}$ & $65 \mathrm{~Hz}$ \\
\hline \multirow{4}{*}{ 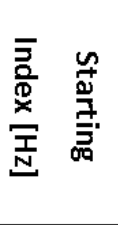 } & $3 \mathrm{~Hz}$ & 2.46 & 1.39 \\
\hline & $8 \mathrm{~Hz}$ & 1.88 & 1.01 \\
\hline & $30 \mathrm{~Hz}$ & N/A & 0.05 \\
\hline & $180 \mathrm{~Hz}$ & $\mathrm{~N} / \mathrm{A}$ & 0.11 \\
\hline
\end{tabular}

\begin{tabular}{|c|c|c|c|}
\hline \multicolumn{2}{|c|}{$\begin{array}{l}\text { Discrimination } \\
\text { matrix }\end{array}$} & \multicolumn{2}{|c|}{$\begin{array}{l}\text { Width of each } \\
\text { band }[\mathrm{Hz}]\end{array}$} \\
\hline & & $27 \mathrm{~Hz}$ & $65 \mathrm{~Hz}$ \\
\hline \multirow{4}{*}{ 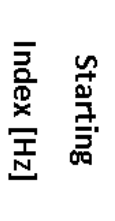 } & $3 \mathrm{~Hz}$ & 0.90 & 0.82 \\
\hline & $8 \mathrm{~Hz}$ & 0.85 & 0.70 \\
\hline & $30 \mathrm{~Hz}$ & $\mathrm{~N} / \mathrm{A}$ & 0.30 \\
\hline & $180 \mathrm{~Hz}$ & $\mathrm{~N} / \mathrm{A}$ & 0.14 \\
\hline
\end{tabular}

A) Values depict the discrimination between Str-GPe of 6 approximately logarithmically spaced bands along the 3-300 $\mathrm{Hz}$ range of the power spectra. The band starts at the starting index and has the specified width (e.g., top left box is the $3-30 \mathrm{~Hz}$ band). The starting index of the band width was simultaneously increased (e.g., for a starting index $=180 \mathrm{~Hz}$, we only tested a width of $65 \mathrm{~Hz}, 180-245 \mathrm{~Hz}$ band). For each band the mean power ( $\mathrm{V}^{2} / \mathrm{Hz}$ ) for each MER was calculated. Then, for each band the absolute value of the difference in the mean power between the Str and GPe was obtained. Finally, the results were normalized by the square root of the sum of the variances of the Str and GPe to yield the discrimination index $\left(\mathrm{V}^{2} / \mathrm{Hz}\right)$. The red represents the frequency bands with the largest absolute differences between the Str and 
GPe for each class (i.e., Parkinson's disease, non-genetic and genetic dystonia). For example, in Parkinson's disease (top left box), maximal Str and GPe discrimination was obtained in two different frequency bands: low frequency $(30-95 \mathrm{~Hz})$ and high frequency $(180-245 \mathrm{~Hz})$ with a value of 2.15 and 1.55 (red color), respectively. B) Similar analysis for the GPe-GPi discrimination matrices.

\section{The Hidden Markov Model}

An HMM procedure was used to estimate the inference states of the electrode at each depth along the trajectory (Fig. 1). We trained three HMMs using 33, 47, and 36 trajectories (i.e., for three classes of disease; can be seen in Fig. 1A) with striatum, GPe, GPi labels as inputs. The input data to the HMM procedure was made up of a sequence of single values based on the features of the MER (Fig. 1C). For example, the features that we used were NRMS, 3-30 Hz, 8-35 Hz, 30-95 Hz and 180-245 Hz from the PSD. For the HMM procedure, a trajectory is a sequence of observations, where at any depth along the trajectory the electrode exists in one of the inference states (Fig. 1E).

\section{Striatum}

6. GPe

\section{GPi}

8. Exit (i.e., white matter)

The HMM inference state sequence was calculated as the most probable sequence beginning with the HMM in striatum (state 1) before the first observation (using the Viterbi algorithm)(Rabiner, 1989). Hence, a typical trajectory inference state starts in the striatum and proceeds through the GPe and GPi consecutively and then to the white matter (exit). However, not all trajectories have clearly defined Str-GPe, GPe-GPi transitions or traversed GPe and GPi; therefore, only trajectories that started $15 \mathrm{~mm}$ above the calculated target and traversed $>7 \mathrm{~mm}$ of GPe and GPi were used for this study.

In the HMM, transitions between states take place according to (1) a fixed probability (i.e., transition matrix and emission matrix) depending solely on the inference state of the electrode at the depth immediately preceding the current inference state and (2) a probability value calculated from the current MER. Note that we used a feed-forward HMM procedure; i.e., as a sequence progresses, it is possible to remain in the same state, but it is not possible to return to a previous state (e.g., from the GPi to the GPe inference state). 
To assess accuracy, the HMM predictions were compared to the electrophysiologist's determination of the location of striatum-GPe, GPe-GPi, and GPi-exit transitions.

Table S3a: The GP PD HMM transition and emission matrices

A

\begin{tabular}{|c|l|l|l|l|l|}
\hline \multicolumn{2}{|l|}{ Transition matrix } & \multicolumn{5}{|l|}{} \\
\cline { 3 - 6 } \multicolumn{2}{|c|}{} & State-1 & State-2 & State-3 & State-4 \\
\hline \multirow{3}{*}{} & Str: State -1 & 0.947 & 0.053 & 0 & 0 \\
\cline { 2 - 6 } & GPe: State-2 & 0 & 0.966 & 0.034 & 0 \\
\cline { 2 - 6 } & GPi: State-3 & 0 & 0 & 0.978 & 0.022 \\
\cline { 2 - 6 } & Exit: State-4 & 0 & 0 & 0 & 1.000 \\
\hline
\end{tabular}

$\mathrm{B}$

\begin{tabular}{|c|c|c|c|c|c|}
\hline \multirow{3}{*}{\multicolumn{2}{|c|}{ Emission matrix }} & \multicolumn{4}{|c|}{ Observations for PD class } \\
\hline & & Low-NRMS & \multirow{3}{*}{$\begin{array}{l}\text { High- } \\
\text { NRMS }\end{array}$} & \multicolumn{2}{|l|}{ High NRMS } \\
\hline & & \multirow[t]{2}{*}{$\begin{array}{l}\text { High mean } \\
\text { Gamma }\end{array}$} & & \multirow{2}{*}{\begin{tabular}{|l|}
$\begin{array}{l}\text { Intermediate } \\
\text { mean Gamma }\end{array}$ \\
Interm. mean \\
$180-245 \mathrm{~Hz}$ \\
\end{tabular}} & $\begin{array}{l}\text { Low mean } \\
\text { Gamma }\end{array}$ \\
\hline & & & & & $\begin{array}{l}\text { High mean } \\
180-245 \mathrm{~Hz}\end{array}$ \\
\hline & & tag-1 & tag-2 & tag-3 & tag-4 \\
\hline \multirow{4}{*}{$\underset{\tilde{a}}{\tilde{\sigma}}$} & Str: State -1 & 0.989 & 0 & 0.011 & 0 \\
\hline & GPe: State-2 & 0.434 & 0.209 & 0.295 & 0.061 \\
\hline & GPi: State-3 & 0.083 & 0.203 & 0.147 & 0.566 \\
\hline & Exit: State-4 & 0.742 & 0.007 & 0.221 & 0.029 \\
\hline
\end{tabular}

(A) The HMM transition matrix. Values depict the probability of a transition between states (or remaining in the current state) for each step of the sequence. (B) The HMM emission matrix. Values depict the probability of each observation given the state. Numerical tags correspond to the four clusters of NRMS, mean $30-95 \mathrm{~Hz}$ and $180-245 \mathrm{~Hz}$ power spectra observations detailed in the table header. States: 1 - Str; 2 - GPe; 3 - GPi; 4 -WM after the $\mathrm{GPi}$; 
PhD Thesis, Dan Valsky

Table S1b: The GP dystonia non-genetic HMM transition and emission matrices

A

\begin{tabular}{|c|l|l|l|l|l|}
\hline \multicolumn{2}{|l}{ Transition matrix } & \multicolumn{5}{|l|}{} \\
\cline { 3 - 6 } \multicolumn{2}{|c|}{} & Class-1 & Class-2 & Class-3 & Class-4 \\
\hline \multirow{2}{*}{} & Str: Class -1 & 0.958 & 0.041 & 0 & 0 \\
\cline { 2 - 6 } & GPe: Class-2 & 0 & 0.959 & 0.041 & 0 \\
\cline { 2 - 6 } & GPi: Class-3 & 0 & 0 & 0.971 & 0.028 \\
\cline { 2 - 6 } & Exit: Class-4 & 0 & 0 & 0 & 1.000 \\
\hline
\end{tabular}

B

\begin{tabular}{|c|c|c|c|c|c|}
\hline \multirow{3}{*}{\multicolumn{2}{|c|}{ Emission matrix }} & \multicolumn{4}{|c|}{ Observations for dystonia non-genetic class } \\
\hline & & Low-NRMS & \multirow{3}{*}{$\begin{array}{l}\text { High- } \\
\text { NRMS }\end{array}$} & \multicolumn{2}{|l|}{ High NRMS } \\
\hline & & \multirow[t]{2}{*}{$\begin{array}{l}\text { Interm. Or } \\
\text { High mean 8- } \\
35 \mathrm{~Hz} \text { band }\end{array}$} & & \multirow[t]{2}{*}{$\begin{array}{l}\text { Low mean 8- } \\
35 \mathrm{~Hz} \text { band }\end{array}$} & $\begin{array}{l}\text { Interm. Or } \\
\text { High mean 8-35 } \\
\text { Hz band }\end{array}$ \\
\hline & & & & & $\begin{array}{l}\text { High mean } \\
3-30 ~ H z\end{array}$ \\
\hline & & tag-1 & tag-2 & tag-3 & tag-4 \\
\hline \multirow{4}{*}{$\frac{\rho}{00}$} & Str: State -1 & 0.991 & 0.004 & 0.002 & 0.002 \\
\hline & GPe: State-2 & 0.353 & 0.081 & 0.524 & 0.041 \\
\hline & GPi: State-3 & 0.200 & 0.248 & 0.203 & 0.348 \\
\hline & Exit: State-4 & 0.731 & 0.127 & 0.123 & 0.017 \\
\hline
\end{tabular}

Similar to Table S1a, but the numerical tags correspond to the four clusters of NRMS, mean 8$35 \mathrm{~Hz}$ and 3-30 Hz power spectra observations detailed in the table header. 
PhD Thesis, Dan Valsky

Table S1c: The GP dystonia genetic HMM transition and emission matrices

A

\begin{tabular}{|c|l|l|l|l|l|}
\hline \multicolumn{2}{|c|}{ Transition matrix } & \multicolumn{5}{|l|}{} \\
\cline { 3 - 6 } & State-1 & State-2 & State-3 & State-4 \\
\hline \multirow{3}{*}{ Str: State -1 } & 0.959 & 0.040 & 0 & 0 \\
\cline { 2 - 6 } & GPe: State-2 & 0 & 0.965 & 0.034 & 0 \\
\cline { 2 - 6 } & GPi: State-3 & 0 & 0 & 0.972 & 0.027 \\
\cline { 2 - 6 } & Exit: State-4 & 0 & 0 & 0 & 1.000 \\
\hline
\end{tabular}

$\mathrm{B}$

\begin{tabular}{|c|c|c|c|c|c|}
\hline \multirow{3}{*}{\multicolumn{2}{|c|}{ Emission matrix }} & \multicolumn{4}{|c|}{ Observations for dystonia genetic class } \\
\hline & & \multirow{2}{*}{$\begin{array}{l}\text { Low-NRMS } \\
\text { High mean 3- } \\
30 \mathrm{~Hz}\end{array}$} & \multirow{2}{*}{$\begin{array}{l}\text { High- } \\
\text { NRMS }\end{array}$} & \multicolumn{2}{|l|}{ High NRMS } \\
\hline & & & & $\begin{array}{l}\text { Intermediate } \\
\text { mean 3-30 } \mathrm{Hz}\end{array}$ & $\begin{array}{l}\text { Low mean 3- } \\
30 \mathrm{~Hz}\end{array}$ \\
\hline & & tag-1 & tag-2 & tag-3 & tag-4 \\
\hline \multirow{4}{*}{$\frac{\mathscr{N}}{\stackrel{0}{0}}$} & Str: State -1 & 0.976 & 0.006 & 0.001 & 0.015 \\
\hline & GPe: State-2 & 0.607 & 0.029 & 0.287 & 0.075 \\
\hline & GPi: State-3 & 0.313 & 0.060 & 0.278 & 0.347 \\
\hline & Exit: State-4 & 0.408 & 0.103 & 0.008 & 0.479 \\
\hline
\end{tabular}

Similar to Table S1a-b, but the numerical tags correspond to the four clusters of NRMS and the mean $3-30 \mathrm{~Hz}$ power spectra observations detailed in the table header.

Values in the emission matrix depict the probability of each observation given the state. The states are defined as in the transition matrix. The four columns correspond to the four observations detailed in the table header as follows:

- All MERs with NRMS $<1.25$ (threshold 1); i.e., below a $25 \%$ increase from the NRMS baseline (equal to 1 due to the normalization) were clustered together (Low-NRMS observation).

- The mean deviation from threshold 1 (i.e. NRMS - 1.25) of the remaining MERs was calculated. Threshold 2 was defined as the threshold 1 plus $25 \%$ of the calculated mean deviation. MERs with a NRMS> threshold 2 were further divided according to their low, 
PhD Thesis, Dan Valsky

intermediate and high mean $3-30 \mathrm{~Hz}, 8-35 \mathrm{~Hz}, 30-95 \mathrm{~Hz}$ and 180-245 Hz oscillatory activity (above or below the median). 
MR image depicting typical anatomical targeting

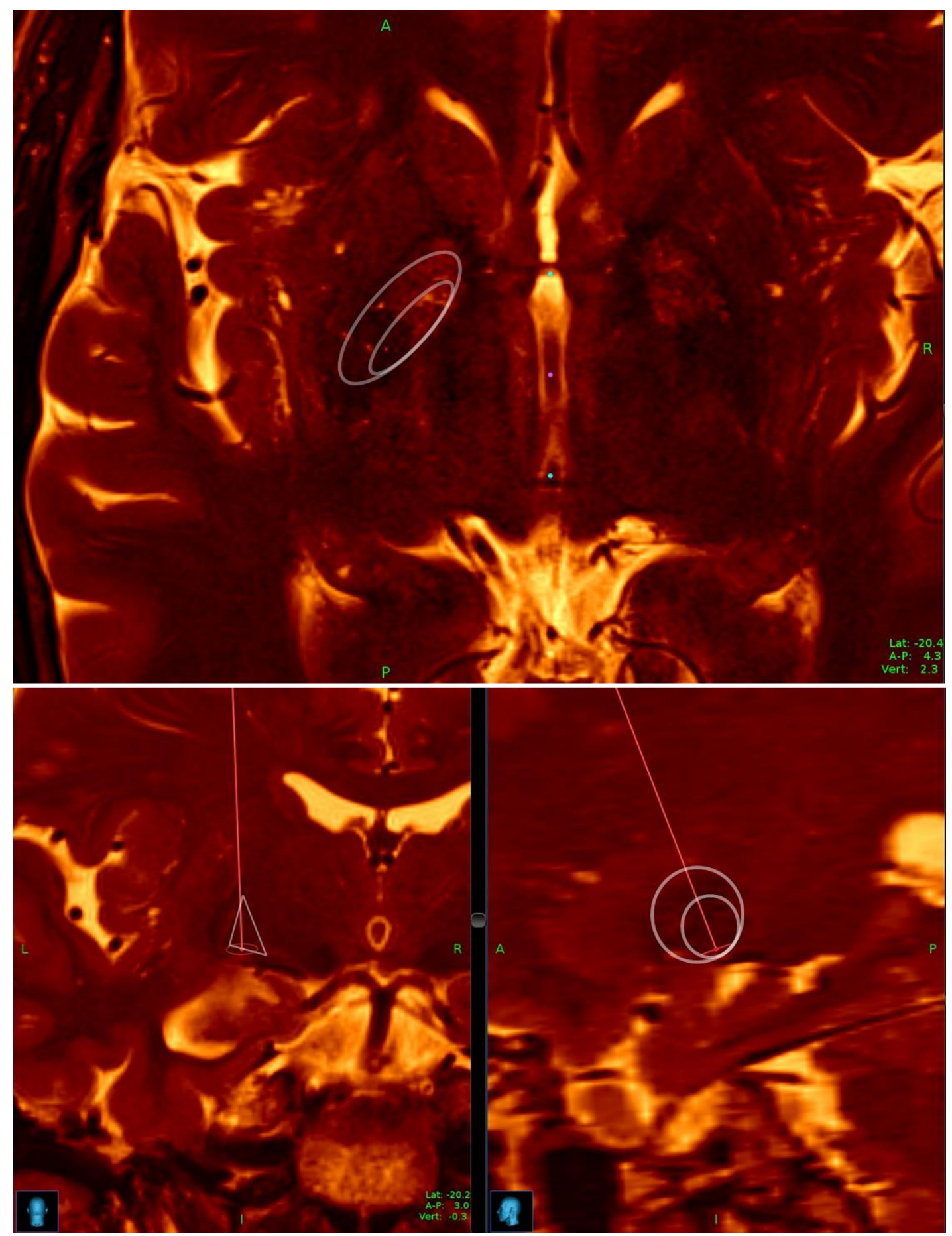

T2 weighted Axial MR image $8 \mathrm{~mm}$ below the ACPC plane with coronal and sagittal reconstructions. The GPe and GPi are shown approximately outlined by the white circle. The red lines represent possible examples of MER tracks that traverse the GPe and GPi. Although the target is the ventro-posterior-lateral GPi, we aimed to have as long a trajectory as possible traversing the GPi through the target point. 


\section{REFERENCE LIST}

Alam M, Sanghera MK, Schwabe K, Lütjens G, Jin X, Song J, von Wrangel C, Stewart RM, Jankovic J, Grossman RG, Darbin O, Krauss JK. 2016. Globus pallidus internus neuronal activity: a comparative study of linear and non-linear features in patients with dystonia or Parkinson's disease. J Neural Transm 123:231-240. doi:10.1007/s00702-015-1484-3

Albin RL, Young AB, Penney JB. 1989. The functional anatomy of basal ganglia disorders. Trends Neurosci 12:366-375. doi:10.1016/0166-2236(89)90074-X

Anderson VC, Burchiel KJ, Hogarth P, Favre J, Hammerstad JP. 2005. Pallidal vs subthalamic nucleus deep brain stimulation in Parkinson disease. Arch Neurol 62:554-60. doi:10.1001/archneur.62.4.554

Aosaki T, Miura M, Suzuki T, Nishimura K, Masuda M. 2010. Acetylcholine-dopamine balance hypothesis in the striatum: An update. Geriatr Gerontol Int. doi:10.1111/j.14470594.2010.00588.x

Bejjani B-P, Dormont D, Pidoux B, Yelnik J, Damier P, Arnulf I, Bonnet A-M, Marsault C, Agid Y, Philippon J, Cornu P. 2000. Bilateral subthalamic stimulation for Parkinson's disease by using three-dimensional stereotactic magnetic resonance imaging and electrophysiological guidance. J Neurosurg 92:615-625. doi:10.3171/jns.2000.92.4.0615

Benabid AL, Chabardes S, Mitrofanis J, Pollak P. 2009. Deep brain stimulation of the subthalamic nucleus for the treatment of Parkinson's disease. Lancet Neurol 8:67-81. doi:10.1016/S14744422(08)70291-6

Benarroch EE. 2012. Effects of acetylcholine in the striatum: Recent insights and therapeutic implications. Neurology 79:274-281. doi:10.1212/WNL.0b013e31825fe154

Benazzouz A, Breit S, Koudsie A, Pollak P, Krack P, Benabid A-L. 2002. Intraoperative microrecordings of the subthalamic nucleus in Parkinson's disease. Mov Disord 17 Suppl 3:S145-9.

Bennett DA, Beckett LA, Murray AM, Shannon KM, Goetz CG, Pilgrim DM, Evans DA. 1996. Prevalence of parkinsonian signs and associated mortality in a community population of older people. N Engl J Med 334:71-6. doi:10.1056/NEJM199601113340202

Bergman H, Wichmann T, DeLong MR. 1990. Reversal of experimental parkinsonism by lesions of the subthalamic nucleus. Science (80- ) 249:1436-1438. doi:10.1126/science.2402638

Bolam JP, Ellender TJ. 2016. Histamine and the striatum. Neuropharmacology. doi:10.1016/j.neuropharm.2015.08.013

Bonsi P, Cuomo D, Martella G, Madeo G, Schirinzi T, Puglisi F, Ponterio G, Pisani A. 2011. Centrality of striatal cholinergic transmission in basal ganglia function. Front Neuroanat 1-9. doi:10.3389/fnana.2011.00006

Bostan AC, Dum RP, Strick PL. 2013. Cerebellar networks with the cerebral cortex and basal ganglia. Trends Cogn Sci. doi:10.1016/j.tics.2013.03.003

Castrioto A, Lhommée E, Moro E, Krack P. 2014. Mood and behavioural effects of subthalamic stimulation in Parkinson's disease. Lancet Neurol 13:287-305. doi:10.1016/S14744422(13)70294-1

Castrioto A, Marmor O, Deffains M, Willner D, Linetsky E, Bergman H, Israel Z, Eitan R, Arkadir D. 2016. Anesthesia reduces discharge rates in the human pallidum without changing the discharge rate ratio between pallidal segments. Eur J Neurosci 44:2909-2913. 
doi:10.1111/ejn.13417

Chen CC, Pogosyan A, Zrinzo LU, Tisch S, Limousin P, Ashkan K, Yousry T, Hariz MI, Brown P. 2006. Intra-operative recordings of local field potentials can help localize the subthalamic nucleus in Parkinson's disease surgery. Exp Neurol 198:214-21. doi:10.1016/j.expneurol.2005.11.019

Choi KS, Noecker AM, Riva-Posse P, Rajendra JK, Gross RE, Mayberg HS, McIntyre CC. 2018. Impact of brain shift on subcallosal cingulate deep brain stimulation. Brain Stimul 11:445-453. doi:10.1016/j.brs.2017.12.001

Cimenser A, Purdon PL, Pierce ET, Walsh JL, Salazar-Gomez AF, Harrell PG, Tavares-Stoeckel C, Habeeb K, Brown EN. 2011. Tracking brain states under general anesthesia by using global coherence analysis. Proc Natl Acad Sci 108:8832-8837. doi:10.1073/pnas.1017041108

Coubes P, Cif L, El Fertit H, Hemm S, Vayssiere N, Serrat S, Picot MC, Tuffery S, Claustres M, Echenne B, Frerebeau P. 2004. Electrical stimulation of the globus pallidus internus in patients with primary generalized dystonia: long-term results. J Neurosurg 101:189-194. doi:10.3171/jns.2004.101.2.0189

Crutcher MD, DeLong MR. 1984. Single cell studies of the primate putamen. Exp Brain Res 53:244258. doi:10.1007/BF00238154

Danish SF, Baltuch GH, Jaggi JL, Wong S. 2008. Determination of subthalamic nucleus location by quantitative analysis of despiked background neural activity from microelectrode recordings obtained during deep brain stimulation surgery. J Clin Neurophysiol 25:98-103. doi:10.1097/WNP.0b013e31816b38dd

Deffains M, Bergman H. 2019. Parkinsonism-related $\beta$ oscillations in the primate basal ganglia networks - Recent advances and clinical implications. Park Relat Disord 59:2-8. doi:10.1016/j.parkreldis.2018.12.015

Deffains M, Iskhakova L, Katabi S, Haber SN, Israel Z, Bergman H. 2016a. Subthalamic, not striatal, activity correlates with basal ganglia downstream activity in normal and parkinsonian monkeys. Elife 5:e16443. doi:10.7554/eLife.16443

Deffains M, Iskhakova L, Katabi S, Haber SN, Israel Z, Bergman H. 2016b. Subthalamic, not striatal, activity correlates with basal ganglia downstream activity in normal and parkinsonian monkeys. Elife 5. doi:10.7554/eLife.16443

DeLong MR. 1971. Activity of pallidal neurons during movement. J Neurophysio/ 34:414-427. doi:10.1152/jn.1971.34.3.414

Dubois B, Pillon B. 1997. Cognitive deficits in Parkinson's disease. J Neurol 244:2-8.

Falkenberg JH, McNames J, Favre J, Burchiel KJ. 2006. Automatic analysis and visualization of microelectrode recording trajectories to the subthalamic nucleus: preliminary results. Stereotact Funct Neurosurg 84:35-44; discussion 44-5. doi:10.1159/000093721

Falkenberg, McNames J, Burchiel K. 2006. Automatic microelectrode recording analysis and visualization of the globus pallidus interna and stereotactic trajectory. Stereotact Funct ....

Filion M, Tremblay L. 1991. Abnormal spontaneous activity of globus pallidus neurons in monkeys with MPTP-induced parkinsonism. Brain Res 547:140-144. doi:10.1016/0006-8993(91)90585-J

Fox SH, Chuang R, Brotchie JM. 2009. Serotonin and Parkinson's disease: On movement, mood, and madness. Mov Disord. doi:10.1002/mds.22473

Fremont R, Tewari A, Angueyra C, Khodakhah K. 2017. A role for cerebellum in the hereditary 
dystonia DYT1. Elife 6. doi:10.7554/eLife.22775

Gerfen CR, Engber TM, Mahan LC, Susel Z, Chase TN, Monsma FJ, Sibley DR. 1990. D1 and D2 dopamine receptor-regulated gene expression of striatonigral and striatopallidal neurons. Science (80- ) 250:1429-1432. doi:10.1126/science.2147780

Graveland GA, Williams RS, Difiglia M. 1985. A Golgi study of the human neostriatum: Neurons and afferent fibers. J Comp Neurol 234:317-333. doi:10.1002/cne.902340304

GuehI D, Cuny E, Ghorayeb I, Michelet T, Bioulac B, Burbaud P. 2009. Primate models of dystonia. Prog Neurobiol. doi:10.1016/j.pneurobio.2008.10.003

Haber SN. 2003. The primate basal ganglia: parallel and integrative networks. J Chem Neuroanat 26:317-30.

Halpern CH, Danish SF, Baltuch GH, Jaggi JL. 2008. Brain Shift during Deep Brain Stimulation Surgery for Parkinson's Disease. Stereotact Funct Neurosurg 86:37-43. doi:10.1159/000108587

Helmich RC. 2018. The cerebral basis of Parkinsonian tremor: A network perspective. Mov Disord 33:219-231. doi:10.1002/mds.27224

Hoshi E, Tremblay L, Féger J, Carras PL, Strick PL. 2005. The cerebellum communicates with the basal ganglia. Nat Neurosci 8:1491-1493. doi:10.1038/nn1544

Hutchison WD, Allan RJ, Opitz H, Levy R, Dostrovsky JO, Lang AE, Lozano AM. 1998.

Neurophysiological identification of the subthalamic nucleus in surgery for Parkinson's disease. Ann Neurol 44:622-628. doi:10.1002/ana.410440407

Hutchison WD, Lang AE, Dostrovsky JO, Lozano AM. 2003. Pallidal neuronal activity: Implications for models of dystonia. Ann Neurol 53:480-488. doi:10.1002/ana.10474

Kemp JM, Powell TP. 1970. The cortico-striate projection in the monkey. Brain 93:525-46.

Ketzef M, Spigolon G, Johansson Y, Bonito-Oliva A, Fisone G, Silberberg G. 2017. Dopamine Depletion Impairs Bilateral Sensory Processing in the Striatum in a Pathway-Dependent Manner. Neuron 94:855-865.e5. doi:10.1016/j.neuron.2017.05.004

Khan MF, Mewes K, Gross RE, Škrinjar O. 2008. Assessment of Brain Shift Related to Deep Brain Stimulation Surgery. Stereotact Funct Neurosurg 86:44-53. doi:10.1159/000108588

Kimura M, Kato M, Shimazaki H. 1990. Physiological properties of projection neurons in the monkey striatum to the globus pallidus. Exp Brain Res 82:672-676. doi:10.1007/BF00228811

Kish L, Palmer MR, Gerhardt GA. 1999. Multiple single-unit recordings in the striatum of freely moving animals: Effects of apomorphine and D-amphetamine in normal and unilateral 6hydroxydopamine-lesioned rats. Brain Res 833:58-70. doi:10.1016/S0006-8993(99)01496-1

Kondabolu K, Roberts EA, Bucklin M, McCarthy MM, Kopell N, Han X. 2016. Striatal cholinergic interneurons generate beta and gamma oscillations in the corticostriatal circuit and produce motor deficits. Proc Natl Acad Sci U S A 113:3159-3168. doi:10.1073/pnas.1605658113

Krause M, Fogel W, Kloss M, Rasche D, Volkmann J, Tronnier V. 2004. Pallidal stimulation for dystonia. Neurosurgery 55:1361-8; discussion 1368-70.

Kühn AA, Trottenberg T, Kivi A, Kupsch A, Schneider GH, Brown P. 2005. The relationship between local field potential and neuronal discharge in the subthalamic nucleus of patients with Parkinson's disease. Exp Neurol 194:212-220. doi:10.1016/j.expneurol.2005.02.010

Lanciego JL, Luquin N, Obeso JA. 2012. Functional neuroanatomy of the basal ganglia. Cold Spring 
Harb Perspect Med 2:a009621. doi:10.1101/cshperspect.a009621

Lemaire N, Hernandez LF, Hu D, Kubota Y, Howe MW, Graybiel AM. 2012. Effects of dopamine depletion on LFP oscillations in striatum are task- and learning-dependent and selectively reversed by L-DOPA. Proc Natl Acad Sci U S A 109:18126-18131. doi:10.1073/pnas.1216403109

Liang L, DeLong MR, Papa SM. 2008. Inversion of dopamine responses in striatal medium spiny neurons and involuntary movements. J Neurosci 28:7537-7547. doi:10.1523/JNEUROSCI.117608.2008

Lozano AM, Hutchison WD. 2002. Microelectrode recordings in the pallidum. Mov Disord 17:S150S154. doi:10.1002/mds.10157

Lozano CS, Ranjan M, Boutet A, Xu DS, Kucharczyk W, Fasano A, Lozano AM. 2018. Imaging alone versus microelectrode recording-guided targeting of the STN in patients with Parkinson's disease. J Neurosurg 1-6. doi:10.3171/2018.2.JNS172186

Mallet N, Ballion B, Le Moine C, Gonon F. 2006. Cortical inputs and GABA interneurons imbalance projection neurons in the striatum of parkinsonian rats. J Neurosci 26:3875-3884. doi:10.1523/JNEUROSCI.4439-05.2006

Maltese M, March J, Bashaw A, Tritsch N. 2019a. Dopamine modulates the size of striatal projection neuron ensembles. doi:10.1101/865006

Maltese M, March JR, Bashaw AG, Tritsch NX. 2019b. Dopamine modulates the size of striatal projection neuron ensembles. bioRxiv 865006. doi:10.1101/865006

McClelland VM, Valentin A, Rey HG, Lumsden DE, Elze MC, Selway R, Alarcon G, Lin J-P. 2016. Differences in globus pallidus neuronal firing rates and patterns relate to different disease biology in children with dystonia. J Neurol Neurosurg Psychiatry 87:958-67. doi:10.1136/jnnp2015-311803

Meidahl AC, Tinkhauser G, Herz DM, Cagnan H, Debarros J, Brown P. 2017. Adaptive Deep Brain Stimulation for Movement Disorders: The Long Road to Clinical Therapy. Mov Disord 32:810819. doi:10.1002/mds. 27022

Menegas W, Bergan JF, Ogawa SK, Isogai Y, Venkataraju KU, Osten P, Uchida N, Watabe-Uchida M. 2015. Dopamine neurons projecting to the posterior striatum form an anatomically distinct subclass. Elife 4. doi:10.7554/eLife.10032

Mink JW. 1996. The basal ganglia: Focused selection and inhibition of competing motor programs. Prog Neurobiol 50:381-425. doi:10.1016/S0301-0082(96)00042-1

Moran A, Bar-Gad I. 2010. Revealing neuronal functional organization through the relation between multi-scale oscillatory extracellular signals. J Neurosci Methods 186:116-129. doi:10.1016/j.jneumeth.2009.10.024

Moran A, Bar-Gad I, Bergman H, Israel Z. 2006. Real-time refinement of subthalamic nucleus targeting using Bayesian decision-making on the root mean square measure. Mov Disord 21:1425-1431. doi:10.1002/mds.20995

Moran A, Bergman H, Israel Z, Bar-Gad I. 2008. Subthalamic nucleus functional organization revealed by parkinsonian neuronal oscillations and synchrony. Brain 131:3395-3409. doi:10.1093/brain/awn270

Moshel S, Shamir RR, Raz A, de Noriega FR, Eitan R, Bergman H, Israel Z. 2013. Subthalamic nucleus long-range synchronization-an independent hallmark of human Parkinson's disease. Front Syst Neurosci 7:79. doi:10.3389/fnsys.2013.00079 
Murray JB. 1996. Depression in Parkinson's disease. J Psychol 130:659-67. doi:10.1080/00223980.1996.9915039

Neumann W-J, Horn A, Ewert S, Huebl J, Brücke C, Slentz C, Schneider G-H, Kühn AA. 2017. A localized pallidal physiomarker in cervical dystonia. Ann Neurol 82:912-924. doi:10.1002/ana.25095

Novak P, Daniluk S, Ellias S a, Nazzaro JM. 2007. Detection of the subthalamic nucleus in microelectrographic recordings in Parkinson disease using the high-frequency (>500 hz) neuronal background. Technical note. J Neurosurg 106:175-179. doi:10.3171/jns.2007.106.1.175

Odekerken VJ, Boel JA, Schmand BA, de Haan Rob J., Figee M, van den Munckhof Pepijn, Schuurman PRR, de Bie Rob M.A., NSTAPS study group F the N study, Group N study, Group F the N study, Group N study, Bie R.M.A. de, Bour L, Contarino MF, Haan R.J. de, Iwan M, Mink MSJ, Munckhof P. van den, Odekerken VJJJVJJJ, Postma M, Schmand BABABA, Scholten MN, Schuurman PRR, Laar T van, Dijk JMC van, Hoffmann CFE, Mosch A, Beute GN, Nijssen PCG, Asseldonk T van, Lenders MWPM, Vugt JPP van, Group N study, NSTAPS study group F the N study. 2016. GPi vs STN deep brain stimulation for Parkinson disease. Neurology 86:755-761. doi:10.1212/WNL.0000000000002401

Okun MS, Fernandez HH, Wu SS, Kirsch-Darrow L, Bowers D, Bova F, Suelter M, Jacobson CE, Wang X, Gordon CW, Zeilman P, Romrell J, Martin P, Ward H, Rodriguez RL, Foote KD. 2009. Cognition and mood in Parkinson's disease in subthalamic nucleus versus globus pallidus interna deep brain stimulation: The COMPARE Trial. Ann Neurol 65:586-595. doi:10.1002/ana.21596

Ostrem JL, Starr PA. 2008. Treatment of dystonia with deep brain stimulation. Neurotherapeutics 5:320-330. doi:10.1016/j.nurt.2008.01.002

Parker JG, Marshall JD, Ahanonu B, Wu YW, Kim TH, Grewe BF, Zhang Y, Li JZ, Ding JB, Ehlers MD, Schnitzer MJ. 2018. Diametric neural ensemble dynamics in parkinsonian and dyskinetic states. Nature 557:177-182. doi:10.1038/s41586-018-0090-6

Petryszyn S, Beaulieu JM, Parent A, Parent M. 2014. Distribution and morphological characteristics of striatal interneurons expressing calretinin in mice: A comparison with human and nonhuman primates. J Chem Neuroanat 59-60:51-61. doi:10.1016/j.jchemneu.2014.06.002

Petryszyn S, Parent A, Parent M. 2018. The calretinin interneurons of the striatum: comparisons between rodents and primates under normal and pathological conditions. J Neural Transm. doi:10.1007/s00702-017-1687-x

Piña-Fuentes D, Beudel M, Little S, van Zijl J, Elting JW, Oterdoom DLM, van Egmond ME, van Dijk JMC, Tijssen MAJ. 2018. Toward adaptive deep brain stimulation for dystonia. Neurosurg Focus 45. doi:10.3171/2018.5.FOCUS18155

Purdon PL, Pierce ET, Mukamel EA, Prerau MJ, Walsh JL, Wong KFK, Salazar-Gomez AF, Harrell PG, Sampson AL, Cimenser A, Ching S, Kopell NJ, Tavares-Stoeckel C, Habeeb K, Merhar R, Brown EN. 2013. Electroencephalogram signatures of loss and recovery of consciousness from propofol. Proc Natl Acad Sci 110:E1142-E1151. doi:10.1073/pnas.1221180110

Purdon PL, Sampson A, Pavone KJ, Brown EN. 2015. Clinical Electroencephalography for Anesthesiologists. Anesthesiology 123:937-960. doi:10.1097/ALN.0000000000000841

Rabiner LR. 1989. A tutorial on hidden Markov models and selected applications in speech recognition. Proc IEEE 77:257-286. doi:10.1109/5.18626

Rodriguez-Oroz MC, Rodriguez M, Guridi J, Mewes K, Chockkman V, Vitek J, DeLong MR, Obeso JA. 
2001. The subthalamic nucleus in Parkinson's disease: somatotopic organization and physiological characteristics. Brain 124:1777-90.

Rolston JD, Englot DJ, Starr PA, Larson PS. 2016. An unexpectedly high rate of revisions and removals in deep brain stimulation surgery: Analysis of multiple databases. Parkinsonism Relat Disord 33:72-77. doi:10.1016/j.parkreldis.2016.09.014

Saenger VM, Kahan J, Foltynie T, Friston K, Aziz TZ, Green AL, van Hartevelt TJ, Cabral J, Stevner ABA, Fernandes HM, Mancini L, Thornton J, Yousry T, Limousin P, Zrinzo L, Hariz M, Marques P, Sousa N, Kringelbach ML, Deco G. 2017. Uncovering the underlying mechanisms and wholebrain dynamics of deep brain stimulation for Parkinson's disease. Sci Rep 7:9882. doi:10.1038/s41598-017-10003-y

Schiff SJ, Dunagan BK, Worth RM. 2002. Failure of single-unit neuronal activity to differentiate globus pallidus internus and externus in Parkinson disease. J Neurosurg 97:119-128. doi:10.3171/jns.2002.97.1.0119

Sharott A, Vinciati F, Nakamura KC, Magill PJ. 2017. A population of indirect pathway striatal projection neurons is selectively entrained to parkinsonian beta oscillations. J Neurosci 37:9977-9998. doi:10.1523/JNEUROSCI.0658-17.2017

Silberstein P, Kühn AA, Kupsch A, Trottenberg T, Krauss JK, Wöhrle JC, Mazzone P, Insola A, Di Lazzaro V, Oliviero A, Aziz T, Brown P. 2003. Patterning of globus pallidus local field potentials differs between Parkinson's disease and dystonia. Brain 126:2597-2608. doi:10.1093/brain/awg267

Singh A, Mewes K, Gross RE, DeLong MR, Obeso JA, Papa SM. 2016. Human striatal recordings reveal abnormal discharge of projection neurons in Parkinson's disease. Proc Natl Acad Sci U S A 113:9629-34. doi:10.1073/pnas.1606792113

Singh A, Papa S. 2019. Aberrant striatal oscillations after dopamine loss in parkinsonian non-human primates. bioRxiv 650770. doi:10.1101/650770

Soares J, Kliem MA, Betarbet R, Greenamyre JT, Yamamoto B, Wichmann T. 2004. Role of external pallidal segment in primate parkinsonism: Comparison of the effects of 1-methyl-4-phenyl1,2,3,6-tetrahydropyridine-induced parkinsionism and lesions of the external pallidal segment. J Neurosci 24:6417-6426. doi:10.1523/JNEUROSCI.0836-04.2004

Sterio D, Zonenshayn M, Mogilner AY, Rezai AR, Kiprovski K, Kelly PJ, Beric A. 2002. Neurophysiological refinement of subthalamic nucleus targeting. Neurosurgery 50:58-67; discussion 67-9.

Tewari A, Fremont R, Khodakhah K. 2017. It's not just the basal ganglia: Cerebellum as a target for dystonia therapeutics. Mov Disord. doi:10.1002/mds.27123

Thompson JA, Yin D, Ojemann SG, Abosch A. 2017. Use of the Putamen as a Surrogate Anatomical Marker for the Internal Segment of the Globus Pallidus in Deep Brain Stimulation Surgery. Stereotact Funct Neurosurg 95:229-235. doi:10.1159/000478105

Valsky D, Blackwell KT, Tamir I, Eitan R, Bergman H, Israel Z. 2020a. Real-time machine learning classification of pallidal borders during deep brain stimulation surgery. J Neural Eng 17:016021. doi:10.1088/1741-2552/ab53ac

Valsky D, Heiman Grosberg S, Israel Z, Boraud T, Bergman H, Deffains M. 2020b. What is the true discharge rate and pattern of the striatal projection neurons in Parkinson's disease and Dystonia? Elife 9. doi:10.7554/eLife.57445 
Valsky D, Marmor-Levin O, Deffains M, Marmor-Levin O, Deffains M, Eitan R, Blackwell KT, Bergman $\mathrm{H}$, Israel Z. 2017. Stop! border ahead: Automatic detection of subthalamic exit during deep brain stimulation surgery 32:70-79. doi:10.1002/mds.26806

Venkatraghavan L, Rakhman E, Krishna V, Sammartino F, Manninen P, Hutchison W. 2016. The effect of general anesthesia on the microelectrode recordings from pallidal neurons in patients with dystonia. J Neurosurg Anesthesiol 28:256-261. doi:10.1097/ANA.0000000000000200

Vidailhet M, Vercueil L, Houeto J-L, Krystkowiak P, Benabid A-L, Cornu P, Lagrange C, Tézenas du Montcel S, Dormont D, Grand S, Blond S, Detante O, Pillon B, Ardouin C, Agid Y, Destée A, Pollak P, French Stimulation du Pallidum Interne dans la Dystonie (SPIDY) Study Group. 2005. Bilateral Deep-Brain Stimulation of the Globus Pallidus in Primary Generalized Dystonia. N Engl J Med 352:459-467. doi:10.1056/NEJMoa042187

Vitek JL, Bakay RAE, Hashimoto T, Kaneoke Y, Mewes K, Zhang JY, Rye D, Starr P, Baron M, Turner R, Delong MR. 1998. Microelectrode-guided pallidotomy: technical approach and its application in medically intractable Parkinson's disease. J Neurosurg 88:1027-1043. doi:10.3171/jns.1998.88.6.1027

Volkmann J, Wolters A, Kupsch A, Müller J, Kühn AA, Schneider G-H, Poewe W, Hering S, Eisner W, Müller J-U, Deuschl G, Pinsker MO, Skogseid I-M, Roeste GK, Krause M, Tronnier V, Schnitzler A, Voges J, Nikkhah G, Vesper J, Classen J, Naumann M, Benecke R, DBS study group for dystonia. 2012. Pallidal deep brain stimulation in patients with primary generalised or segmental dystonia: 5-year follow-up of a randomised trial. Lancet Neurol 11:1029-1038. doi:10.1016/S1474-4422(12)70257-0

Wang DD, de Hemptinne C, Miocinovic S, Ostrem JL, Galifianakis NB, San Luciano M, Starr PA. 2018. Pallidal Deep-Brain Stimulation Disrupts Pallidal Beta Oscillations and Coherence with Primary Motor Cortex in Parkinson's Disease. J Neurosci 38:4556-4568. doi:10.1523/JNEUROSCI.043118.2018

Weaver FM, Follett KA, Stern M, Luo P, Harris CL, Hur K, Marks WJ, Rothlind J, Sagher O, Moy C, Pahwa R, Burchiel K, Hogarth P, Lai EC, Duda JE, Holloway K, Samii A, Horn S, Bronstein JM, Stoner G, Starr PA, Simpson R, Baltuch G, De Salles A, Huang GD, Reda DJ, CSP 468 Study Group. 2012. Randomized trial of deep brain stimulation for Parkinson disease: Thirty-sixmonth outcomes. Neurology 79:55-65. doi:10.1212/WNL.0b013e31825dcdc1

Welter M-L, Grabli D, Karachi C, Jodoin N, Fernandez-Vidal S, Brun Y, Navarro S, Rogers A, Cornu P, Pidoux B, Yelnik J, Roze E, Bardinet E, Vidailhet M. 2015. Pallidal activity in myoclonus dystonia correlates with motor signs. Mov Disord 30:992-996. doi:10.1002/mds.26244

Wichmann T. 2018. Pathophysiologic Basis of Movement Disorders. Prog Neurol Surg 33:13-24. doi:10.1159/000480718

Wichmann T, DeLong MR. 2003. Pathophysiology of Parkinson's disease: The MPTP primate model of the human disorderAnnals of the New York Academy of Sciences. New York Academy of Sciences. pp. 199-213. doi:10.1111/j.1749-6632.2003.tb07477.x

Zaidel A, Spivak A, Grieb B, Bergman H, Israel Z. 2010. Subthalamic span of $\beta$ oscillations predicts deep brain stimulation efficacy for patients with Parkinson's disease. Brain 133:2007-2021. doi:10.1093/brain/awq144

Zaidel A, Spivak A, Shpigelman L, Bergman H, Israel Z. 2009. Delimiting subterritories of the human subthalamic nucleus by means of microelectrode recordings and a hidden Markov model. Mov Disord 24:1785-1793. doi:10.1002/mds.22674 